Merci d'utiliser le titre suivant lorsque vous citez ce document :

van Tongeren, F., J. Beghin et S. Marette (2009-12-17), « Cadre d'analyse coût-avantages pour l'évaluation des mesures non tarifaires s'appliquant aux échanges agroalimentaires », Éditions OCDE, Paris. http://dx.doi.org/10.1787/218778445444

\title{
Cadre d'analyse coût- avantages pour l'évaluation des mesures non tarifaires s'appliquant aux échanges agroalimentaires
}

\author{
Frank van Tongeren, \\ John Beghin, \\ Stéphane Marette
}

La version originale de ce document a été publiée comme suit :

van Tongeren, F., J. Beghin and S. Marette (2009-11-01), "A Cost-Benefit Framework for the Assessment of Non-Tariff Measures in Agro-Food Trade", OECD Food, Agriculture and Fisheries Papers, No. 21, OECD Publishing, Paris. http://dx.doi.org/10.1787/220613725148 


\section{Avant-propos}

Le présent document élabore un cadre conceptuel destiné à évaluer les coûts et avantages associés aux mesures non tarifaires afin de procéder à une analyse comparative fondée sur les faits de différentes approches réglementaires. Il a été préparé par Frank van Tongeren (Secrétariat de l'OCDE), John Beghin (Université d'État de l'Iowa), et Stéphan Marette (INRA) et a bénéficié des contributions de Joanna Komorowska (Secrétariat de l'OCDE).

Mots clefs : Agriculture dans le commerce international, économie de régulation, information et qualité du produit, organisations internationales du commerce, normalisation et comparabilité, politiques des échanges 


\section{Table des matières}

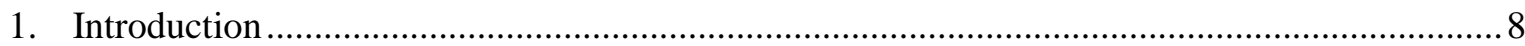

2. Une taxonomie des défaillances et des imperfections du marché ..............................................11

2.1. Défaillances du marché ayant une incidence sur les consommateurs .............................11

2.2. Défaillances du marché ayant une incidence sur les producteurs..................................... 12

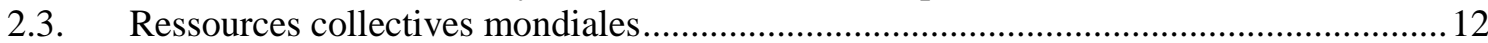

2.4. Surveillance imparfaite et autres défaillances des pouvoirs publics................................13

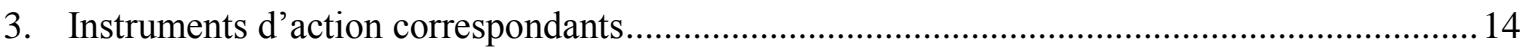

3.1. La classification élargie des MNT établie par la MAST ….............................................. 14

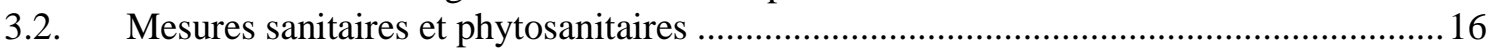

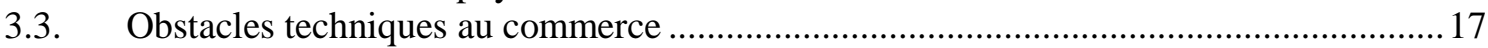

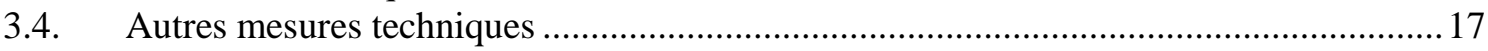

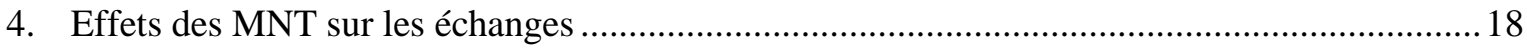

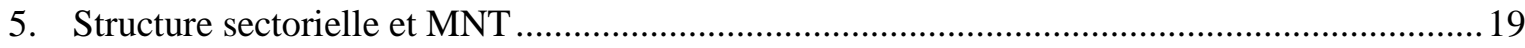

6. Utilisation de l'analyse coûts-avantages et d'autres méthodes ...............................................20

7. Analyse coûts-avantages : une approche modulaire........................................................22

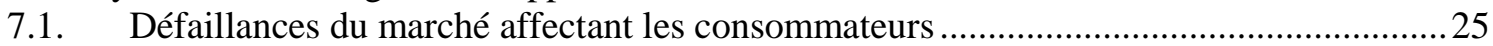

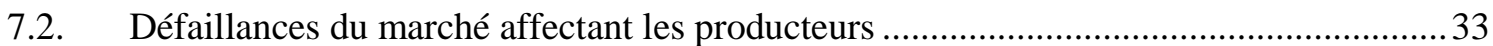

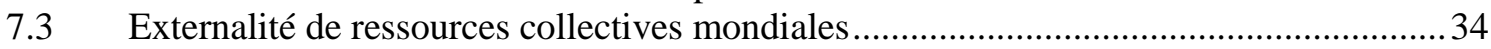

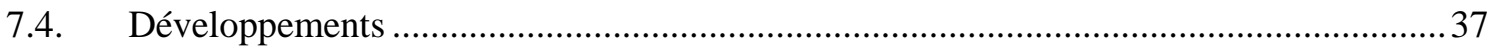

8. Avancées récentes en matière d'évaluation des défaillances du marché

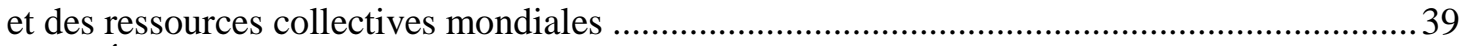

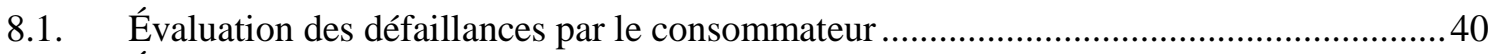

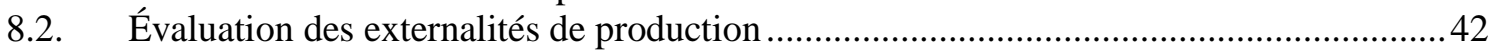

8.3. Valeur accordée aux ressources collectives mondiales .....................................................

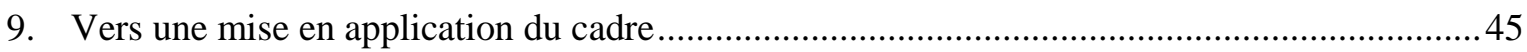

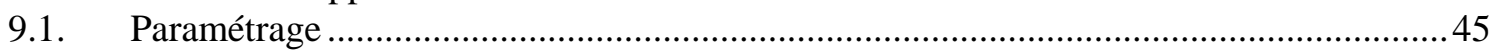

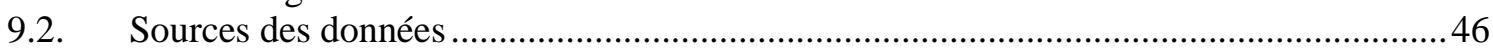

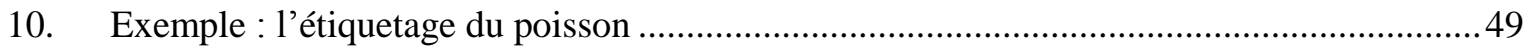

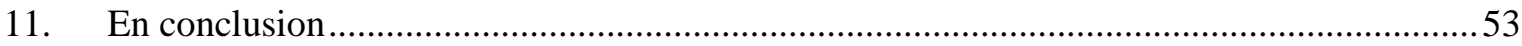




\section{RÉSUMÉ}

Les pouvoirs publics sont de plus en plus appelés à répondre à toute une série de préoccupations de la société dans des domaines aussi divers que l'environnement, le bien-être animal et la sécurité alimentaire. Des mesures correctives sont attendues lorsque le marché n'existe pas ou est défaillant, générant des résultats inefficients. Si la solution apportée par le marché n'est pas satisfaisante, les pouvoirs publics disposent de divers moyens d'intervention, qui comprennent des mesures réglementaires, fiscales ou des subventions. Dans certains cas, répondre à ces préoccupations requiert des mesures purement locales ou nationales qui n'ont guère d'incidences sur les échanges ou sur la politique commerciale. Mais dans le cas de biens faisant l'objet d'échanges internationaux, les mesures non tarifaires (MNT) deviennent un instrument d'action de plus en plus important, surtout dans le contexte actuel d'intégration du commerce et de suppression des obstacles classiques au commerce, comme les droits de douane et les contingents.

Les importations peuvent introduire des espèces envahissantes comme des agents pathogènes, ravageurs ou mauvaises herbes qui sont étrangères à l'écologie d'un pays. Les partenaires commerciaux n'ont pas nécessairement tous les mêmes normes de sécurité alimentaire ou les mêmes capacités institutionnelles pour les faire appliquer, ce qui peut conduire à importer des produits alimentaires qui ne satisfont pas aux exigences nationales. Un contrôle imparfait et incomplet aux frontières, si tant est qu'il ait lieu, aggrave les risques pour l'environnement ou la santé. Dans les pays où les droits de la propriété sont mal définis, les échanges peuvent aussi encourager la production non viable de certains biens destinés à l'exportation, conduisant à une détérioration des ressources communes mondiales.

L'évaluation des effets économiques des MNT présente de grandes difficultés. De nombreuses mesures techniques peuvent freiner le commerce mais améliorer le bien-être en réduisant les externalités négatives (ex. diminution du risque d'importer des ravageurs ou des maladies) ou les asymétries de l'information (ex. étiquette fournissant au consommateur des informations sur le produit). D'autres mesures peuvent accroître les échanges dans la mesure où elles renforcent la demande d'un bien en fournissant de meilleures informations sur ce bien ou en améliorant ses caractéristiques.

Les coûts d'efficience des MNT sont bien moins évidents que les pertes de bien-être associées aux tarifs douaniers et aux quantités. Les mesures non tarifaires n'intègrent pas nécessairement les inefficiences économiques associées aux obstacles commerciaux traditionnels, à moins qu'elles n'opèrent une discrimination entre les différentes sources d'approvisionnement. Elles peuvent constituer les mesures ayant le moins d'effets de restriction sur les échanges qui existent compte tenu des imperfections du marché. À priori, on ne peut pas dire clairement si la réglementation a un impact sur les échanges, ou si la suppression de mesures non tarifaires associées préjudiciables aux échanges 
permettrait de réaliser des gains d'efficience supérieurs aux pertes induites par une réglementation moins puissante.

Ce rapport élabore un cadre d'analyse unifié qui permet d'évaluer les coûts et les avantages des mesures pour les acteurs de la chaîne d'approvisionnement: consommateurs, producteurs et pouvoirs publics nationaux, ainsi que fournisseurs étrangers et même, le cas échéant, consommateurs et pouvoirs publics étrangers. L'évaluation englobe les effets sur les échanges, car les mesures non tarifaires influent sur les échanges en étant à l'origine d'effets externes, mais elle examine aussi d'autres effets. Ce cadre d'analyse permet de comparer différentes méthodes employées pour concevoir les mesures et de distinguer leurs effets sur les échanges et sur le bien-être. Par exemple, une interdiction des importations (ou une norme dissuasive) visant à empêcher un produit comportant une caractéristique indésirable d'entrer sur le marché peut être comparée à son autorisation assortie de la condition que le produit étranger soit clairement identifiable ( $e x$. par son étiquetage).

Ce cadre d'analyse a pour caractéristique centrale de distinguer les consommateurs (ou les producteurs) qui attachent de l'importance à certains effets externes négatifs et positifs et/ou attributs du produit de ceux qui y sont indifférents. L'importance pour les consommateurs concernés d'éviter la caractéristique indésirable du produit est une variable déterminante de l'évaluation coûts-avantages des mesures qui remédient aux défaillances touchant les consommateurs. Il est difficile de mesurer cette importance de manière empirique, mais les progrès récents de l'économie de la consommation sont prometteurs. Concernant les producteurs, l'intérêt d'éviter une défaillance est directement lié à la valeur de la perte de production susceptible de se produire s'il n'est pas remédié à cette défaillance.

Le cadre d'évaluation coûts-avantages est, pour l'essentiel, un modèle d'équilibre partiel modulaire, qui intègre des fonctions de demande et d'offre et qui peut être calibré en fonction de données empiriques pour permettre de calculer les effets sur le bien-être économique. La conception modulaire rend l'approche suffisamment souple pour y ajouter des calculs annexes. À titre d'exemple, on peut ajouter aisément des estimations détaillées des coûts induits par la surveillance et l'application des mesures, même si, pour l'heure, de telles estimations ne sont pas effectuées. Le rapport mentionne également, sans les étudier en détail, plusieurs paramètres supplémentaires pouvant être intégrés dans l'analyse, comme des normes non dissuasives auxquelles les entreprises nationales comme étrangères s'efforcent de se conformer en y consacrant d'importantes ressources financières, et dont les consommateurs peuvent dans une certaine mesure apprécier le degré de conformité. Les autres paramètres possibles incluent l'entrée et la sortie d'entreprises compte tenu des coûts de conformité fixes et variables.

La méthodologie proposée s'applique à la comparaison de différents choix politiques tels que la mise en place de normes, de contrôles aux frontières et d'étiquetage, dans un contexte international. Elle contribue à analyser les mesures non tarifaires de manière plus complète qu'en s'intéressant aux seuls effets commerciaux.

L'application du cadre à des problématiques spécifiques devra tenir compte des limites de la méthode exposée dans ce document, surtout s'agissant de questions relatives à la santé humaine. Dans de tels cas, des méthodes de substitution sont examinées succinctement et pourraient être employées. Les résultats empiriques dépendront de la configuration des paramètres et de la qualité des données utilisées. Pour évaluer la fiabilité des résultats et souligner les limites, on peut recourir à une analyse de sensibilité approfondie faisant intervenir diverses hypothèses, un intervalle de valeurs de paramètres 
et différents scénarios. Il peut être plus pertinent de déterminer si une conclusion est fiable avec un intervalle de paramètres plausibles que de procéder à une seule estimation du bien-être.

L'approche comparative proposée des MNT permet d'identifier différentes manières de réagir à un problème de réglementation donné. En énumérant systématiquement les coûts et les avantages pour les différents agents économiques intéressés, il est possible de suivre une approche reposant sur des preuves et formant une base solide d'échanges mutuels et d'identification des solutions les moins coûteuses. 


\section{Cadre d'analyse coûts-avantages pour l'évaluation des mesures non tarifaires s'appliquant aux échanges agroalimentaires}

\section{Introduction}

La Reinheitsgebot (loi allemande sur la pureté de la bière) datant d'une loi bavaroise de 1516 est un exemple bien connu de règles qui décrivent comment un produit destiné à la consommation humaine doit être fabriqué pour pouvoir être commercialisé. La Reinheitsgebot, motivée en partie par des préoccupations concernant la sécurité alimentaire, visait à s'assurer que les ingrédients employés étaient adéquats1. Quatre siècles et demi plus tard, l'Allemagne a dû renoncer à imposer ses règles de brassage de la bière pour cause de libéralisation des échanges sur le marché intérieur de l'UE. Depuis l'abrogation de la norme allemande unilatérale, les consommateurs peuvent déguster des bières d'autres fournisseurs européens et non européens, en plus de la bière toujours produite selon la Reinheitsgebot.

Cette anecdote historique illustre joliment les questions traitées dans ce rapport. Les États adoptent et appliquent des règlements dans les secteurs de l'agriculture et de la production alimentaire afin de sauvegarder les intérêts de la société lorsque les marchés non réglementés ne produisent pas les résultats souhaités. Ces règlements portent souvent sur des aspects liés à la santé humaine ; d'autres traitent de problèmes d'environnement et de bien-être animal associés à la production agricole. Tant que le règlement concerne un bien (ou un service) non exportable, la conception optimale des mesures réglementaires n'a pas à prendre en compte l'intérêt de parties étrangères. C'était largement le cas au $\mathrm{XVI}^{\mathrm{e}}$ siècle sur le marché bavarois de la bière, qui était très localisé avec très peu d'échanges transfrontières. Toutefois, lorsque le produit peut être exporté, des mesures aux frontières et à l'intérieur des frontières sont généralement prises pour faire en sorte que les variétés importées répondent aux exigences nationales. Interdire tous les produits non conformes sur le marché intérieur, comme sur le marché allemand de la bière avant le jugement de la Cour européenne de justice en 1987, est un exemple extrême d'une telle mesure non tarifaire (MNT).

Avec l'accélération de l'intégration internationale, les échanges sont de plus en plus générateurs d'effets externes, et les pouvoirs publics réagissent à la nécessité de sauvegarder les intérêts nationaux en adoptant un large éventail de mesures non tarifaires

1. Outre les ingrédients du brassage (orge, houblon et eau), la loi réglementait également le conditionnement et les prix pour la vente de bière. La Bavière a posé comme condition préalable à l'unification allemande voulue par Otto von Bismarck en 1871 l'acceptation nationale de la Reinheitsgebot. Elle ne fut appliquée à l'ensemble du territoire de l'Allemagne qu'en 1907. En Allemagne, la controverse sur les boissons qui méritent véritablement le nom de «bière » se poursuit aujourd'hui encore. Un autre objectif poursuivi était de restreindre l'utilisation de blé dans le brassage en vue de l'affecter à la production de pain. 
(Levine et d'Antonio, 2003). Les importations peuvent introduire des espèces envahissantes comme des agents pathogènes, ravageurs ou mauvaises herbes qui sont étrangères à l'écologie d'un pays (CABI, divers). Les partenaires commerciaux n'ont pas nécessairement tous les mêmes normes de sécurité alimentaire ou les mêmes capacités institutionnelles pour les faire appliquer, ce qui peut conduire à importer des produits alimentaires qui ne satisfont pas aux exigences nationales. Un contrôle imparfait et incomplet aux frontières, si tant est qu'il ait lieu, aggrave les risques pour l'environnement ou la santé. Dans les pays où les droits de la propriété sont mal définis, les échanges peuvent aussi encourager la production non viable de certains biens destinés à l'exportation, conduisant à une détérioration des ressources communes mondiales (Chichilnisky, 1994).

Dans certains cas où les échanges sont directement à l'origine d'importants effets externes, il peut être envisageable de les retreindre, mais certaines mesures sont plus efficaces que d'autres pour lutter contre les effets externes pernicieux. De nombreux obstacles techniques aux échanges peuvent freiner le commerce mais améliorer le bienêtre en présence d'externalités négatives ou d'asymétries de l'information. D'autres mesures peuvent accroître les échanges dans la mesure où elles renforcent la demande d'un bien en fournissant de meilleures informations sur ce bien ou en améliorant ses caractéristiques.

Les différentes formes de MNT s'accompagnent de coûts et d'avantages différents selon la composante de la société concernée. Par exemple, l'interdiction des bières non conformes sur le marché allemand a profité à certains producteurs de bière nationaux en évinçant la concurrence étrangère. Elle a peut être aussi évité aux consommateurs d'ingérer des ingrédients nocifs, mais les a également privés de l'accès aux bières étrangères, dès lors que la technologie nécessaire pour pouvoir exporter ce produit sur de grandes distances était disponible.

Le présent rapport élabore un cadre de prise en compte systématique des coûts et des avantages économiques des MNT. En examinant spécifiquement les avantages que les MNT peuvent procurer en remédiant à différents types de défaillances du marché, cette approche se démarque sensiblement des études récentes consacrées aux obstacles non tarifaires $^{2}$ qui adoptent souvent une vision étroitement mercantiliste des pertes en termes d'échanges (ex. Otsuki et al., 2001). Le cadre d'analyse coûts-avantages élaboré dans ce document permet d'évaluer, sous l'angle économique, différentes réponses aux mêmes défaillances du marché. Trois grandes catégories de défaillances sont étudiées: 1) défaillances touchant les consommateurs, comme des informations imparfaites relatives à la sécurité alimentaire, mais aussi les préoccupations des consommateurs à l'égard des méthodes de production ; 2) défaillances touchant les producteurs, comme les épizooties ; 3) problèmes affectant les ressources collectives mondiales, généralement liés à la conservation d'écosystèmes de grande valeur. Enfin, nous abordons aussi le problème du manque de contrôle concernant les normes alimentaires.

L'instrument élaboré est un cadre unifié qui permet d'apprécier les effets économiques des MNT conçues pour remédier à ces différents types de défaillances du marché. Ce cadre a pour caractéristique centrale de faire la distinction entre les consommateurs (ou producteurs) qui sont touchés par la défaillance du marché et ceux

2. Les obstacles non tarifaires forment un sous-ensemble des mesures non tarifaires, concept plus large utilisé dans ce rapport, car on ne peut pas déterminer d'emblée si une mesure donnée constitue effectivement un obstacle aux échanges. 
qui ne le sont pas, et d'en déduire une méthode pour mesurer, de manière empirique, les avantages et les coûts associés aux MNT pour ces différents groupes. En pratique, la distinction entre ces deux catégories peut être malaisée. Concernant les consommateurs, cette approche repose sur les enseignements de l'économie empirique moderne appliquée à la consommation et, pour les producteurs, elle intègre les enseignements d'études épidémiologiques.

Le cadre inclut différents types de consommateurs classés en fonction de leurs préoccupations à l'égard des effets externes négatifs et positifs et/ou des attributs du produit. Ces préoccupations peuvent dépendre des informations dont ils disposent sur ces attributs. Si les produits nationaux et étrangers n'ont pas les mêmes caractéristiques, certaines MNT, comme l'étiquetage obligatoire, peuvent révéler des informations manquantes et conduire à une demande différenciée des consommateurs pour les variétés nationales et étrangères. L'offre étrangère peut aussi être un vecteur d'externalités négatives dans la production, comme l'introduction d'espèces envahissantes. Le cadre proposé tient compte de ces effets et des MNT associées en mesurant leurs répercussions sur le coût de production des producteurs nationaux concernés. Ce cadre étudie également les ressources collectives mondiales.

Ce cadre est modulaire, permettant d'ajouter ou de soustraire de nouveaux éléments de la structure principale, accompagnés de calculs détaillés en annexe, sans devoir modifier la logique générale de l'approche.

L'objectif global de ce travail, défini dans la note exploratoire OCDE (2007a), est de déterminer l'importance des MNT dans les échanges agricoles et alimentaires et de quantifier leur impact économique. En s'attachant à mettre en place un cadre d'évaluation économique d'une sélection de mesures non tarifaires prises par les pouvoirs publics dans le secteur agroalimentaire de pays de l'OCDE, ce rapport constitue la première étape vers une approche empirique qui constituera une base solide pour les échanges mutuels et l'identification des solutions les moins onéreuses. Il présente un cadre conceptuel qui facilite l'évaluation comparative des différentes approches visant à remédier à une défaillance ou à une imperfection donnée du marché par des mesures réglementaires qui influent sur les échanges internationaux. Ce travail conceptuel est complété par plusieurs études de cas empiriques sélectionnées au moyen d'une procédure fondée sur des données et qui mettent en lumière les forces et les faiblesses du cadre conceptuel.

La structure de ce rapport est la suivante. Nous examinons dans un premier temps les principales formes de défaillances et d'imperfections du marché (section 2). La section 3 décrit les moyens d'action associés aux défaillances recensées dans la section 2; la section 4 présente leurs effets sur les échanges. La section 5 analyse les transformations potentielles dans les structures du marché induites par les MNT et les coûts de conformité associés. La section 6 présente la modélisation retenue. Les autres sections donnent des indications sur l'utilisation pratique du cadre proposé. La section 7 passe en revue les évolutions récentes en matière d'évaluation des effets externes. La section 8 explique comment mettre en œuvre ce cadre, tandis que la section 9 en fournit l'illustration. La dernière section propose une feuille de route pour les travaux futurs. Plusieurs annexes fournissent des informations complémentaires sur les MNT (annexe 1) et sur la modélisation retenue (annexe 2). 


\section{Une taxonomie des défaillances et des imperfections du marché}

Cette section aborde les principales formes d'imperfections et de défaillances du marché qui sont pertinentes pour les MNT. Des marchés imparfaits et défaillants conduisent à des résultats non efficients, qui constituent un important motif d'intervention des pouvoirs publics. De nombreuses MNT tentent de remédier aux effets externes. Des externalités surviennent lorsque le bien ou le service d'un agent dépend des choix opérés par d'autres agents qui ne tiennent pas compte de ces effets externes dans leurs décisions. Par conséquent, une externalité génère des coûts ou des avantages pour un agent qui ne sont pas reflétés dans les valorisations sur le marché. Il est utile de caractériser une externalité par son point d'impact afin d'organiser la réflexion. Lorsqu'un coût ou un avantage externe survient dans la sphère de la consommation, il sera désigné par le concept d'externalité de consommation, alors que les externalités de production désignent celles dont l'effet se manifeste dans la sphère de la production. Prenons l'exemple des résidus chimiques nocifs issus de la production, mais dont l'effet potentiel sur la santé sera ressenti par les consommateurs ; ce type d'externalité sera donc considéré comme une externalité de consommation.

Les autres imperfections du marché auxquelles les MNT tentent de répondre ont trait aux conséquences d'informations asymétriques (un partenaire d'une transaction est mieux informé que l'autre) ou imparfaites (toutes les conséquences ne sont pas connues). On peut aisément classer les problèmes d'information selon qu'ils affectent les consommateurs ou les producteurs. Ils peuvent jouer un rôle dans la surveillance des prescriptions et des règlements par les pouvoirs publics.

La taxonomie commence par recenser les défaillances ayant une incidence sur les consommateurs ; le rapport étudie ensuite leurs pendants pour les producteurs, à savoir les défaillances liées à la production. La section suivante examine les répercussions sur les ressources collectives mondiales, avant d'aborder certains aspects liés à un contrôle imparfait.

\subsection{Défaillances du marché ayant une incidence sur les consommateurs}

Externalités touchant les consommateurs: ce scénario implique souvent une externalité négative subie par les agents économiques et qui ne résulte pas de la production ou de la consommation du bien. Les consommateurs sont touchés par l'effet externe qui est indépendant de leur propre panier d'achat. Si un bien génère une pollution au cours de sa production ou de sa consommation, il risque d'avoir des répercussions sur d'autres personnes qui ne sont absolument pas concernées par sa production ou sa consommation. Les inquiétudes des consommateurs concernant le bien-être animal en sont un exemple : certains consommateurs (ou plutôt « citoyens ») s'interrogent sur les méthodes de production; leur bien-être en est affecté, quelle que soit leur décision de consommer ou non de la viande d'animaux élevés dans certaines conditions ${ }^{3}$. On peut distinguer les consommateurs du pays importateur et ceux du pays exportateur, dans la mesure où ils peuvent être touchés différemment.

3. Ce cas correspond aux dommages causés par l'externalité qui peuvent être « séparés » de la consommation du marché. L'externalité influe sur le bien-être du consommateur représentatif, mais pas directement sur sa décision de consommer sur le marché. Par convention, la théorie économique considère que les citoyens sont des consommateurs dès lors qu'ils ne sont pas producteurs. 
Informations asymétriques sur la santé, la sécurité ou la valeur nutritionnelle : ce type d'imperfection est associé à l'achat ou à la consommation du bien par un consommateur final. Le consommateur tire un avantage de la consommation du bien, mais supporte également un coût ou bénéficie d'un avantage qu'il ne connaît pas précisément du fait de l'impact sur sa santé. Les coûts sociaux perçus et réels du bien sont donc différents. Si le producteur est correctement informé des caractéristiques du bien, une situation d'information asymétrique prévaut. Certains attributs liés à l'expérience ou à la croyance ne sont pas connus du consommateur au moment de l'achat et peuvent réduire (dans le cas d'ingrédients nocifs) ou augmenter (dans le cas de bienfaits nutritionnels) la valeur du bien. D'autres, en revanche, peuvent être nocifs et présenter un danger pour le consommateur. Les épidémies d'e-coli et de salmonelle dont certains consommateurs du pays importateur ou exportateur n'avaient pas connaissance sont des exemples récents de cas où des informations asymétriques peuvent être associées à une sous-évaluation des risques sanitaires.

\subsection{Défaillances du marché ayant une incidence sur les producteurs}

Externalités touchant les producteurs : des effets externes se produisent lorsque le processus de production d'un bien est altéré par des facteurs externes autres que les prix. Par exemple, la pollution de l'eau peut avoir un impact sur les produits issus de la pêche. La pollution est provoquée par la consommation, la production ou les échanges dans d'autres régions ou par l'environnement proprement dit, comme dans le cas de la rouille du soja véhiculée aux États-Unis par les ouragans. Elle provoque une baisse de la production ou une augmentation des coûts de production, soit par perte d'efficience (baisse des rendements agricoles), soit par tentative de remédier aux effets externes (application de fongicides afin d'éliminer certains champignons). Ces événements peuvent être non rivaux (toute une région est frappée par une épidémie de fièvre aphteuse) ou d'ordre privé (un seul producteur est touché par la pollution). Parfois, les externalités peuvent être positives, comme de nouvelles connaissances technologiques importées non rivales en ce sens que la plupart des producteurs nationaux peuvent y accéder.

Informations asymétriques au niveau de la production : les producteurs, à l'instar des consommateurs, peuvent être eux aussi victimes d'informations asymétriques et acheter des intrants dont les attributs sont nocifs (ex. : une maladie transmise par les semences et qui contamine un exploitant agricole peut entraîner des pertes).

\subsection{Ressources collectives mondiales}

Les ressources collectives mondiales désignent les ressources perçues comme appartenant à la communauté (mondiale) et nécessitant une gestion collective. Ce sont des ressources collectives à accès libre, pour lesquelles les droits de propriété sont mal définis ou ne le sont pas du tout. Les exemples de problèmes concernant ces ressources collectives incluent l'utilisation non durable des ressources forestières, l'épuisement des stocks de poissons à cause de la surpêche et la production agricole ayant un impact écologique négatif. Il n'est pas nécessaire d'être soi-même consommateur d'un bien spécifique pour être touché par l'externalité. Toutefois, choisir des produits certifiés comme respectant les ressources collectives peut procurer un bénéfice aux consommateurs. Les écolabels et le commerce équitable sont des exemples bien connus de mesures qui procurent des avantages perçus aux consommateurs soucieux de sauvegarder les ressources collectives. 
Bien que le traitement analytique des questions afférentes aux ressources communes soit souvent étroitement lié au traitement des externalités qui touchent les consommateurs, il est utile d'en faire une catégorie à part car de plus en plus de frictions commerciales entre l'OCDE et les économies en développement trouvent leurs origines dans des problèmes tenant aux ressources communes et les pratiques durables suscitent un intérêt croissant. Les échanges jouent souvent un rôle central : un bien est importé d'un pays caractérisé par des problèmes de ressources collectives ou des pratiques non durables. Le pays importateur peut prendre une mesure non tarifaire afin de tenter de remédier au problème touchant les ressources collectives dans le pays où il s'approvisionne.

\subsection{Surveillance imparfaite et autres défaillances des pouvoirs publics}

Dans la pratique, la mise en œuvre des politiques réglementaires existantes est souvent incomplète et insuffisamment contrôlée. À cet égard, les pouvoirs publics sont coupables de ne pas en faire assez, ce qu'on désigne parfois par le concept de défaillance par omission. Les capacités institutionnelles limitées pour surveiller et appliquer les règlements exigent parfois des interventions supplémentaires ou peuvent nécessiter des mesures qui, si la surveillance était parfaite, ne seraient pas justifiées par l'amélioration du bien-être. Les efforts visant à remédier aux défaillances institutionnelles peuvent avoir de fortes répercussions sur les échanges et entraîner des mesures coûteuses. L'incapacité de détecter et de circonscrire rapidement une épidémie de fièvre aphteuse ou d'ESB peut entraîner un effondrement des échanges si les partenaires ferment leurs frontières à titre de mesure d'urgence. Si les ressources institutionnelles affectées aux contrôles aux frontières sont limitées, un pays peut choisir de désigner un seul port d'entrée pour les importations de certains produits alimentaires, mesure qui peut renchérir les coûts des échanges. D'autres mesures peuvent être davantage axées sur la coopération dès lors qu'elles sont planifiées, surtout dans les relations Nord-Sud. Par exemple, la coordination des stratégies, comme la certification des exportateurs du Sud par les pays importateurs du Nord, dégage les ressources supplémentaires qui font défaut dans le pays exportateur (ex. l'aide apportée par l'UE aux entreprises de conditionnement de viande d'Amérique latine afin de se conformer aux normes phytosanitaires et de sécurité alimentaire de l'UE).

Dans de nombreux pays, la traçabilité complète et la surveillance parfaite échappent aux autorités. Même les cadres réglementaires les plus perfectionnés des pays de l'OCDE ne peuvent pas empêcher entièrement les épidémies de salmonelle et d'e-coli. En théorie, une supervision optimale devrait mettre en parallèle les coûts et les avantages escomptés des activités de supervision, ce qui signifie souvent qu'une surveillance complète et une prévention totale seraient excessivement coûteuses. En règle générale, la capacité de réglementer efficacement le secteur agroalimentaire est plus faible dans les pays à bas revenu à qui l'expérience institutionnelle et les ressources financières font défaut. Si les moyens consacrés aux inspections et aux contrôles sont limités, la «défaillance par omission » qui en résulte (les pouvoirs publics n'en font pas assez) exacerbera les problèmes de sécurité alimentaire et les externalités liées aux ressources mondiales, car les consommateurs ne disposent pas d'informations sur le processus de fabrication et les caractéristiques des aliments importés qu'ils achètent. Dans ce contexte, les normes privées peuvent jouer en partie ce rôle (Fulponie, 2006, Garcia Martinez et al., 2007). 


\section{Instruments d'action correspondants}

Un large éventail de mesures permet de s'attaquer aux imperfections du marché évoquées ci-dessus, mais le présent rapport se concentre sur les mesures susceptibles d'avoir une incidence sur les échanges internationaux, en distinguant les mesures non tarifaires. L'Équipe d'appui multiorganisations $(\mathrm{MAST})^{4}$, qui réalise les travaux techniques pour le compte d'un groupe de personnalités éminentes chargées par les directeurs généraux de la CNUCED et de l'OMC d'avancer sur le dossier des obstacles non tarifaires, a élaboré la définition suivante des MNT :

Les mesures non tarifaires (MNT) sont des mesures autres que les droits de douane ordinaires qui sont susceptibles d'avoir un effet économique sur les échanges internationaux de biens et de modifier les quantités échangées, les prix ou les deux (MAST, 2008).

Cette définition est large et éclaire aussi peu que l'ancienne catégorie des obstacles non tarifaires, car les mesures non tarifaires et les obstacles non tarifaires sont définis par dérivation comme étant des mesures autres que les droits de douane.

Cette définition large des MNT n'implique pas de jugement a priori sur leur effet économique réel, leur efficacité pour atteindre différents objectifs stratégiques ou leur statut juridique au regard du cadre juridique de l'OMC ou d'autres accords commerciaux. Dans le contexte des travaux que l'OCDE consacre aux MNT, cette définition a été restreinte (OCDE, 2007a) en excluant les mesures qui ont une incidence directe sur les prix et les quantités (contingents, contingents tarifaires, entreprises commerciales d'État, licences d'importation et mesures antidumping), afin de se concentrer sur la catégorie de mesures la moins étudiée ayant un impact indirect sur les prix et/ou les quantités en influant sur d'autres attributs des biens vendus, en général des mesures réglementaires. La section suivante illustre les mesures qui relèvent de cette catégorie.

\subsection{La classification élargie des MNT établie par la MAST}

L'équipe MAST a élaboré un nouveau système de classification des MNT qui étend considérablement la classification TRAINS utilisée à l'origine par la CNUCED afin de répertorier les mesures commerciales (voir la section 8 sur le système TRAINS-WITS). Cette nouvelle classification se caractérise notamment par le fait qu'elle établit des catégories distinctes pour les mesures SPS et OTC et qu'elle inclut les entraves liées aux procédures (qui ont trait à la mise en œuvre des mesures et non aux mesures proprement dites). La classification est actuellement testée par la CNUCED et par le CCI via des études pilotes dans sept pays, et devrait être révisée à la lumière de l'expérience acquise à

4. Les institutions membres de la MAST en juillet 2008 étaient les suivantes : Organisation des Nations Unies pour l'alimentation et l'agriculture (FAO), Fonds monétaire international (FMI), Centre du commerce international CNUCED/OMC (CCI), Organisation de coopération et de développement économiques (OCDE/TAD), Conférence des Nations Unies sur le commerce et le développement (CNUCED), Organisation des Nations Unies pour le développement industriel (ONUDI), Banque mondiale (BM), Organisation mondiale du commerce (OMC). Les observateurs sont : Commission européenne (CE), Commission du commerce international des États-Unis (USITC), ministère de l'Agriculture des États-Unis (USDA). La CNUCED et la Banque mondiale assurent la coordination conjointe de la MAST. La MAST rend compte au Groupe de personnalités éminentes créé par le directeur général de la CNUCED. Elle a remis son premier rapport en juillet 2008. 
l'occasion de ces études pilotes ${ }^{5}$. Ce système vise essentiellement à intégrer tout l'éventail des mesures existantes; il se garde donc, dans toute la mesure du possible, de formuler des hypothèses préalables sur les conséquences potentielles des mesures et, en ce sens, ne constitue pas une grille d'analyse. Les principales catégories de la nouvelle classification MAST sont les suivantes (la liste complète intégrant des sous-catégories supplémentaires figure à l'annexe 1) :

(A) Mesures sanitaires et phytosanitaires

(B) Obstacles techniques au commerce

(C) Autres mesures techniques

(D) Mesures de contrôle des prix

(E) Mesures de contrôle des quantités

(F) Mesures para-tarifaires

(G) Mesures financières

(H) Mesures anticoncurrentielles

(I) Mesures relatives aux exportations

(J) Mesures concernant les investissements liées au commerce

(K) Restrictions relatives à la distribution des produits

(L) Restrictions des services après-vente

(M) Subventions

(N) Restrictions relatives aux marchés publics

(O) Propriété intellectuelle

(P) Règles d'origine

Les principales catégories de la classification des obstacles liés aux procédures sont les suivantes :

- Caractère arbitraire ou incohérent

- Comportement discriminatoire favorisant des producteurs ou des fournisseurs spécifiques

- Inefficience ou obstruction

- Absence de transparence

- Problèmes juridiques

- Frais ou droits excessivement élevés (ex. droits de timbre, frais facturés au titre d'essais ou d'autres services rendus)

Les mesures qui relèvent des catégories (A) à (C) sont les plus pertinentes dans le cadre du présent rapport. Elles ont un lien relativement évident avec les imperfections du

5. Les études de cas concernent les pays suivants : Brésil, Chine, Inde, Ouganda, Philippines, Thaïlande et Tunisie. 
marché traitées précédemment. Ces grandes catégories représentent l'essentiel des interventions dont le nombre augmente au niveau mondial (Beghin, 2006) et ont des effets d'affectation analogues. Elles alourdissent les coûts de production en augmentant le coût marginal ou les coûts fixes, et ont tendance à perturber les structures sectorielles. Les mesures de ce type peuvent aussi accroître la demande de produits de la part des consommateurs en améliorant leur qualité ou en réduisant les asymétries d'information concernant le produit ciblé. Certaines de ces mesures touchent à la fois les producteurs et les consommateurs. De nombreuses mesures appartenant aux catégories (A) à (C) poursuivent des objectifs portant sur les capacités institutionnelles. Elles visent parfois à remédier aux carences des partenaires commerciaux (défaillance par omission de la part du pays exportateur); dans d'autres circonstances, elles nécessitent de disposer de capacités institutionnelles nationales importantes pour mettre en ouvre les stratégies retenues. Bien que les ressources et les stades de production concernés varient selon le type d'obligation, la plupart de ces mesures augmentent le coût de production, soit à la marge, soit en pesant sur les coûts fixes.

Les effets des mesures de contrôle des prix (catégorie D) sont relativement bien compris, et les moyens d'action relevant de la catégorie (E) ont été examinés en détail à l'occasion de l'analyse des contingents, des contingents tarifaires et de leur administration (voir par exemple OCDE, 2002a, 2002b ; Boughner, de Gorter et Sheldon, 2000). Les mesures paratarifaires (catégorie F) peuvent être considérées comme des instruments conventionnels de politique fiscale dont l'incidence est simple à déterminer. Cette catégorie d'instruments pourrait aisément être ajoutée au cadre proposé ci-dessous. Les catégories restantes $(\mathrm{G})$ à $(\mathrm{P})$ sont elles aussi importantes, mais ne peuvent pas être facilement intégrées au cadre de modélisation proposé. Toutefois, les obstacles tenant aux procédures peuvent être convertis en coût commercial et exprimés en équivalents tarifaires dans la plupart des cas ; en principe, ils pourraient être intégrés au cadre proposé ci-dessous.

\subsection{Mesures sanitaires et phytosanitaires}

Certaines mesures SPS figurant dans la catégorie (A) s'attaquent à l'asymétrie de l'information entre producteurs et consommateurs de produits porteurs d'attributs de croyance afin de faire en sorte que les consommateurs soient informés sur les produits qu'ils achètent et sachent qu'ils sont sains pour leur santé ou pour l'environnement. Les normes et obligations visent les processus et les attributs des produits. Les mesures SPS ciblent également les externalités potentielles de production introduites par des espèces envahissantes ou des maladies infectieuses. La plupart des mesures SPS de la catégorie (A) entraînent une hausse du coût marginal de production du fait des coûts supplémentaires encourus pour satisfaire aux obligations. En outre, certaines d'entre elles peuvent également accroître la demande en fournissant des informations aux consommateurs.

Certaines mesures sanitaires et phytosanitaires sont directement liées au commerce en tant que vecteur des externalités. Par exemple, certaines interventions visent les fournisseurs étrangers sur une base géographique. La catégorie A280, restrictions géographiques, constitue fondamentalement une interdiction des importations basée sur l'origine. L'hétérogénéité réglementaire, à savoir le fait que les normes de l'exportateur ne sont pas conformes aux exigences de l'importateur, entraîne de nombreuses mesures sanitaires et phytosanitaires. Les exigences de certification (A310) régissent le cadre institutionnel dans le pays ou à l'étranger afin de garantir le respect des normes. Plusieurs 
sous-catégories tentent de remédier au manque de réciprocité dans les systèmes de certification et de réglementation lorsque certaines normes, internationales notamment, ne sont pas reconnues (voir les sous-catégories qui relèvent de la catégorie A320, manque de reconnaissance).

\subsection{Obstacles techniques au commerce}

Ce sont des règlements et des normes qui portent sur les caractéristiques techniques de produits. Comme dans le cas des mesures sanitaires et phytosanitaires, il existe des normes volontaires applicables aux processus et aux attributs des produits (B100 et sous-catégories correspondantes). Les normes régissant les processus et les produits modifient les courbes de coûts en alourdissant les coûts de production, et peuvent influer positivement sur la demande en réduisant l'asymétrie de l'information entre fournisseurs et consommateurs. Des produits plus sains sont vraisemblablement plus attrayants pour tout ou partie des consommateurs. À la différence des mesures sanitaires et phytosanitaires, les obstacles techniques au commerce n'incluent pas d'interdictions explicites des importations originaires de certains pays ou de certaines régions.

Les règlements techniques (B200 et sous-catégories correspondantes) couvrent les normes et les prescriptions obligatoires. Là encore, ils englobent les normes relatives aux produits et aux processus, et ont vraisemblablement pour effet de décaler l'offre vers la gauche et la demande vers la droite. Ces règlements ciblent l'asymétrie de l'information et le comportement opportuniste des fournisseurs au regard des attributs de croyance. Cette catégorie inclut la traçabilité et l'origine des matières premières, ainsi que les limites de tolérance pour les résidus et les restrictions d'utilisation de certaines substances (sous-catégorie B230). Les règlements relatifs aux OGM constituent une autre sous-catégorie de B200, comme les obligations en matière de préservation de l'identité et spécifiques à l'environnement, qui portent sur les externalités induites par la production et/ou sur les enjeux relatifs aux ressources collectives.

La sous-catégorie B300 porte sur l'évaluation de conformité, la certification et le test des produits; elle couvre également les cas dans lesquels le manque de reconnaissance des procédures de certification peut entraver les échanges. Ce dernier aspect relève davantage des défaillances institutionnelles ou stratégiques évoquées ci-dessus.

\subsection{Autres mesures techniques}

La troisième catégorie de MNT englobe les mesures et obligations qui, sans cadrer parfaitement avec les deux premières, leur ressemblent beaucoup à des fins d'analyse selon certains critères. La sous-catégorie $\mathrm{C} 100$ couvre les inspections avant expédition afin de s'assurer de la conformité des produits, ce qui peut permettre de remédier à la défaillance par omission susmentionnée; la catégorie C200 couvre les formalités douanières ne relevant pas des catégories (A) et (B). Enfin, la catégorie C900 désigne les mesures techniques non mentionnées ailleurs qui ne sont pas clairement motivées par des imperfections du marché. 


\section{Effets des MNT sur les échanges}

Les approches économiques généralement suivies pour analyser les MNT établissent souvent un effet unidirectionnel de pertes commerciales sans faire clairement le lien entre ces pertes et le bien-être. Le projet s'emploie dès le départ à dépasser cette approche et à tenir dûment compte des répercussions sur le bien-être des mesures ciblant les externalités et les imperfections du marché. De nombreuses MNT peuvent restreindre les échanges mais améliorer le bien-être en présence des externalités négatives ou de l'asymétrie d'information évoquées plus haut. D'autres mesures peuvent accroître les échanges dans la mesure où elles renforcent la demande d'un bien en fournissant de meilleures informations sur ce bien ou en améliorant ses caractéristiques (Maertens et al. (2007) ; Maertens et Swinnen (2009)). Face à des risques de maladies, des MNT bien conçues peuvent permettre des échanges en nombre limité, alors qu'en l'absence de mesures comme des contrôles plus sévères aux frontières ou la restriction des importations aux produits d'un pays ou d'une région spécifique, les échanges peuvent être nuls.

Les ouvrages existants supposent implicitement que l'harmonisation des MNT améliore le bien-être. Une fois harmonisés, ces règlements peuvent réduire le coût unitaire de production grâce à des économies d'échelle et garantir la libre circulation des marchandises sur un marché unifié, comme cela s'est produit au sein de l'UE. Néanmoins, le choix des consommateurs peut aussi être restreint si les biens harmonisés étaient initialement différents et si les goûts varient d'un pays à l'autre (Moenius, 2006). Les avantages et inconvénients de l'harmonisation ne sont pas encore parfaitement connus et l'analyse des travaux sur le sujet ne permet pas de dégager de consensus sur les mesures à prendre (Barrett et Yang, 2001 ; Casella, 1996 et Gandal et Shy, 2001). Des discordances apparaissent entre la théorie et les ouvrages pratiques sur l'harmonisation des MNT. Les évaluations empiriques adoptent souvent une vision mercantiliste en se focalisant sur les pertes en termes d'échanges (ex. Otsuki et al., 2001) au lieu d'examiner leurs effets potentiels d'amélioration du bien-être.

Étant donné que le commerce peut être vecteur de transmission d'une externalité, certaines MNT s'attaquent directement aux échanges considérés comme étant la cause de l'externalité, en imposant par exemple une restriction ou une interdiction dans le cas d'une infection. Plusieurs auteurs ont examiné ces restrictions dans le contexte d'épizooties (Wilson et Anton, 2006 ; Lee et Paarlbergh, 1998 ; Pendell et al., 2007 et d'autres).

Les effets des MNT sur les échanges ont été analysés en détail en recourant aux équations de gravité. De nombreuses analyses de gravité sont généralement de large portée (plusieurs produits ou secteurs, pays et mesures). Cette démarche permet de procéder à un examen global d'hypothèses générales, comme les effets de restriction ou au contraire d'accroissement des échanges induits par les MNT ou l'impact de l'harmonisation. L'équation de gravité a également été appliquée pour étudier certaines questions spécifiques comme les mesures prises par l'UE dans le dossier de l'aflatoxine (Otsuki et al., 2001). La plupart des études détectent l'existence d'effets de restriction des échanges induits par les mesures techniques en appliquant divers indicateurs (niveaux, comptages, EAV, différentiels de prix). Au-delà des effets de restriction bien connus de nombreuses mesures SPS et OTC, des effets d'amplification des échanges ont également été recensés, souvent grâce à l'harmonisation et à la mise en commun des normes, dans les unions douanières et pour certains produits et certaines mesures (Disdier et al., 2008 ; Fontagné et al., 2005 ; Henry de Frahan et Vancauteren, 2006 ; et Moenius, 1999, 2006). 
Quelques études concluent à l'absence d'effets des mesures techniques sur les échanges dans certains secteurs (ex. Fontagné et al., 2005) et pour les mesures harmonisées (Czubala et al., 2007). Les effets des MNT ont également été étudiés au moyen de modèles de simulation d'équilibre partiel et général, le plus souvent en les paramétrant en tant qu'équivalents tarifaires dans les fonctions de demande à l'importation (ou d'offre à l'exportation). Voir OCDE (2008b) pour un examen des approches quantitatives.

\section{Structure sectorielle et MNT}

Le respect des MNT entraîne des coûts pour les producteurs, tant variables (activités supplémentaires) que fixes (investissements supplémentaires), qui peuvent d'avoir d'importantes répercussions sur la structure du secteur. Si le respect des normes et règlements implique de lourds investissements irrécupérables une fois engagés, les économies d'échelle deviennent une caractéristique majeure du secteur. Les coûts irrécupérables liés aux MNT peuvent se transformer en barrière à l'entrée et devenir un déterminant essentiel de la structure du secteur. Toutes les entreprises ne s'aligneront pas sur les nouvelles normes et la structure d'un secteur peut être profondément modifiée par les nouvelles règles de production imposées à l'exportateur, ce qui peut avoir des conséquences sur la participation au marché, notamment dans les pays à bas revenu. Ces disparités dans la capacité à se conformer aux normes entraînent souvent un dualisme dans le secteur concerné par le nouveau cadre réglementaire. On assiste à l'émergence d'un segment moderne et dynamique, alors que les producteurs plus petits sont marginalisés et desservent un marché intérieur informel, quittent le marché ou deviennent salariés d'entreprises plus grandes (Rau et van Tongeren 2007 ; Maertens et Swinnen 2006, et d'autres).

Il est important de connaître l'effet précis des MNT sur les coûts fixes et variables : des variations des coûts variables se traduisent par des révisions des prix, et si toutes les entreprises subissent la même hausse de leurs coûts variables pour se conformer aux MNT, l'effet sur la compétitivité n'est pas évident. Toutefois, les investissements irrécupérables ne sont pas pris en compte dans la fixation de prix optimums par les entreprises et ont des effets plus indirects sur les prix du marché par le biais de l'entrée et de la sortie d'entreprises. Seules les entreprises suffisamment productives pour 'surmonter l'obstacle' que représentent les coûts fixes d'entrée sur le marché pourront exporter (Melitz, 2003).

Mesurer le coût de conformité présente de grandes difficultés. Différentes méthodes sont employées: enquêtes auprès des entreprises (ex. Wilson et Otsuki, 2004b), comparaisons de prix (Yue et al., 2006; Ferrantino 2006), comptabilité analytique (ex. Grothe et al., 2000), estimations économétriques (Antle, 2000 ; Maskus et al., 2005).

Le principal problème à traiter lorsqu'on mesure le coût de conformité tient au choix du point de référence correct. Dans certains cas, les producteurs auraient procédé aux adaptations requises pour se conformer aux exigences des importateurs, même en l'absence des normes, ou engagent des transformations qui ne sont pas directement nécessaires pour respecter les règles. On peut se demander, dans ces circonstances, si les coûts directs de ces modifications peuvent être considérés comme des coûts de conformité. Des difficultés supplémentaires surviennent si les exportateurs desservent plusieurs marchés où les exigences sont différentes, ce qui peut entraîner des coûts de conformité hétérogènes d'un marché à l'autre et alourdir le coût global de conformité. L'harmonisation peut réduire ces coûts. 


\section{Utilisation de l'analyse coûts-avantages et d'autres méthodes}

L'objectif de quantifier les effets économiques des mesures non tarifaires peut être appréhendé comme une évaluation systématique des coûts et des avantages d'un changement hypothétique de politique. La question est la suivante : «quels sont les coûts et les avantages probables d'un changement de politique ? » La stratégie actuelle peut consister à ne pas réglementer ni intervenir sur le marché (ne rien faire). Le problème généralement posé par cette évaluation est qu'il est impossible de mesurer avec précision une partie des coûts et des avantages pertinents - tout simplement parce qu'un changement de politique est hypothétique et qu'on ne dispose pas d'observations empiriques qui renseignent sur les réactions des consommateurs et des producteurs au nouveau contexte.

Les ouvrages consacrés à l'analyse coûts-avantages ont élaboré plusieurs approches visant à remédier à ce problème spécifique de mesure, et elles sont souvent employées pour l'élaboration des politiques. Plusieurs pays de l'OCDE, dont le Canada, certains États membres de l'UE et les États-Unis fournissent des instructions officielles sur la marche à suivre pour réaliser une analyse coûts-avantages exhaustive dans un but d'élaboration des politiques, surtout dans le domaine des réformes de la réglementation. Au niveau de l'UE, il existe des directives officielles sur les évaluations coûts-avantages dans plusieurs domaines pertinents dans l'ensemble de l'UE. Plusieurs raisons expliquent pourquoi l'analyse coûts-avantages est largement employée (OCDE, 2006c). Premièrement, elle fournit une base de décision rationnelle en contraignant le décideur à se demander quels sont les gagnants et les perdants d'un changement de politique. Deuxièmement, elle exige que toute politique soit considérée comme une option parmi d'autres. Troisièmement, l'analyse coûts-avantages contribue à déterminer l'échelle optimale de mise en œuvre de la politique, en choisissant l'option qui maximise les bénéfices nets. Enfin, une analyse coûts-avantages tient explicitement compte de la dimension temporelle pour évaluer les décisions d'investissement, grâce au processus d'actualisation.

Profondément ancrée dans la théorie de l'économie du bien-être, l'analyse coûts-avantages tient compte des préférences individuelles ${ }^{6}$. Elle traduit la notion théorique des préférences en une mesure pratique des choix du consommateur, par le biais du concept de la propension à payer (ou de la propension à recevoir). La section 8 ci-dessous analyse les progrès récents dans la mesure de la propension à payer (PAP) et des autres méthodes employées.

Malgré son attrait, plusieurs contraintes peuvent entraver la réalisation d'une analyse coûts-avantages exhaustive. Ces contraintes vont des efforts nécessaires pour réunir l'information à la survenue d'effets qui ont un impact peu clair ou indirect sur les préférences des consommateurs. Cependant, le principal obstacle est sans doute la difficulté de monétiser les effets de mesures qui sauvent des vies. Les consommateurs peuvent être réticents à donner une valeur monétaire à ou à exprimer leur propension à payer pour une vie sauvée. Deux solutions existent pour contourner l'impossibilité de fixer un prix pour une vie humaine.

6. Certains peuvent considérer que cet aspect est une faiblesse plutôt qu'une force de l'analyse coûts-avantages, parce qu'elle implique que les préférences comptent même si ceux qui les manifestent ne sont pas bien informés. Pour un examen complet, voir OCDE, 2006c. 
La première méthode substitutive, l'analyse coûts-efficacité, évite de devoir estimer les avantages représentés par une vie sauvée. Elle compare les diverses options possibles en évaluant leurs coûts ainsi qu'une mesure quantitative unique mais non monétaire de l'efficacité. L'exemple classique est celui du coût de différentes mesures par vie statistique sauvée (voir par exemple Morall, 2003). Cette approche accorde davantage d'importance à l'évaluation des coûts. Si elle s'appuie uniquement sur le calcul des coûts budgétaires, elle risque de sous-estimer les coûts économiques totaux d'un changement de politique.

La deuxième méthode substitutive est l'approche dite QALY (années de vie en bonne santé), généralement employée dans le secteur médical et dans celui de la santé publique. Les coûts d'autres politiques sont comparés aux effets sur la santé, mesurés selon deux critères : la qualité de vie (morbidité) et la durée de vie (mortalité). Il existe plusieurs méthodes pour estimer les coûts de la mortalité et de la morbidité et pour évaluer, en termes monétaires, les avantages d'une action de l'État engendrant une diminution du risque sanitaire. Les méthodes statistiques dose-effet servent à estimer la réduction des risques. Avec la méthode dite du capital humain, une valeur est accordée au risque réduit de décès prématuré sur la base d'une évaluation des flux actualisés des revenus du travail. Pour un individu d'un âge donné, la valeur de la vie statistique prolongée par une réglementation correspond à la somme actualisée des espérances mathématiques de revenu de cette personne (Freeman, 1993). Avec la méthode du coût de la maladie, une valeur est accordée à la réduction de morbidité permise par la réglementation, sur la base des frais médicaux estimés et des pertes de productivité dues à la maladie (Buzby et al., 1996). Les coûts d'opportunité issus de l'investissement dans des activités qui réduisent le risque sont inclus dans la valeur de la réduction de la maladie (Landelfeld et Seskin, 1982). Le principal inconvénient de ces approches est leur incapacité de refléter les réactions des consommateurs dans la demande et leur impact indirect sur les producteurs. Les coûts estimés au moyen des méthodes QALY n'ont pas d'équivalent en termes d'ajustements de la demande liés à la réaction des consommateurs. Par conséquent, elles ne peuvent pas prendre en compte les réactions des prix du marché et leur incidence sur les producteurs et les consommateurs.

La méthode QALY est généralement utilisée dans le domaine de la médecine et de la santé publique, tandis que le modèle PAP (propension à payer) est le plus employé dans les analyses relatives aux transports et à l'environnement. On peut se référer à Hammitt (2002) pour une analyse et un comparatif des principaux avantages et inconvénients sousjacents des deux approches. En principe, ces deux méthodes peuvent être utilisées pour analyser les MNT, en fonction du cas d'espèce. Le cadre d'analyse coûts-avantages assorti de mesures PAP est d'autant plus efficient que les défaillances du marché liées aux MNT concernent des aspects qualitatifs qui ne peuvent pas se traduire par des maladies à court terme clairement identifiables (comme la préférence pour le bien-être animal, l'origine des produits, la protection d'espèces en danger, la préférence négative pour les OGM).

Quelle que soit la méthode employée, la précision et la portée de la mesure des coûts et des avantages se heurteront toujours à des limites. L'utilisation de modèles différents qui expliquent le même phénomène peut conduire à des résultats différents. Une façon de traiter le problème consiste à évaluer chaque modèle séparément, puis à comparer leurs résultats, éventuellement en tenant compte de la probabilité que les prévisions s'avèrent justes avec chacun des modèles (voir par exemple Conseil du Trésor du Canada, 2007). 


\section{Analyse coûts-avantages : une approche modulaire}

Cette section présente un cadre d'évaluation systématique des coûts et des avantages des mesures non tarifaires qui aborde les effets externes et les imperfections de marché décrits plus haut. Le cadre théorique est conçu pour être appliqué avec des données empiriques, de manière à faciliter une analyse quantitative coûts-avantages. Cette section a pour objectif d'exposer les grandes lignes d'une approche commune et ses principes sous-jacents. Nous ne nous penchons pas ici sur la totalité des effets potentiels, le cadre pouvant aisément s'étendre dans plusieurs directions pour analyser des problèmes commerciaux spécifiques. Un élément qui n'est pas analysé ici mais qui peut être important dans la pratique concerne les coûts liés à l'administration, au suivi et à l'application.

Le cadre proposé ici tend vers une analyse du bien-être qui peut être calibrée et quantifiée à partir de situations réelles de politique économique. Le modèle permet d'effectuer une analyse comparative des effets sur le bien-être des différentes approches et des différentes mesures non tarifaires pour remédier au même type d'imperfections de marché ; nous avons choisi de l'appliquer à trois situations : le libre-échange, la mise en place d'une norme prohibitive ou une interdiction d'importation, et le libre-échange avec étiquetage.

Le cadre comprend des «modules » de calcul des coûts et des avantages pour (a) les consommateurs nationaux, (b) les producteurs nationaux, (c) les États (où a lieu la consommation), et (d) les producteurs étrangers. Pour des raisons de simplicité, les consommateurs et les États étrangers ne sont pas inclus dans l'analyse. En outre, les différents acteurs de la chaîne d'approvisionnement (agriculteurs, industries de transformation, distributeurs, etc.) sont rassemblés en une seule étape de production qui représente l'offre. Ces conventions influent très souvent sur les résultats, mais elles sont conservées ici pour permettre une présentation claire. Le cadre fait la distinction entre les coûts et les avantages externes pour différents agents qui résultent des défaillances, et les coûts et avantages pour ces mêmes agents induits par les mesures prises pour remédier à ces défaillances.

Le cadre d'analyse distingue notamment les agents qui attachent de l'importance à une imperfection de marché donnée de ceux qui y sont indifférents. Du côté des consommateurs, cela suppose une opposition entre les utilisateurs qui affichent une préférence pour certaines caractéristiques du bien concerné (ou qui préfèrent éviter certaines caractéristiques indésirables) et ceux qui ne s'en préoccupent pas. Cette distinction peut sembler difficile à opérer dans la pratique, mais elle est essentielle pour obtenir un résultat exact. Les progrès récents de l'économie de la consommation, et notamment de l'économie expérimentale, offrent les moyens de segmenter les consommateurs en différents groupes en observant leur comportement de choix. De même, pour les producteurs, il s'agit d'opérer une distinction entre ceux affectés par les externalités de production et ceux qui ne le sont pas. La présentation suppose un minimum de détails techniques, et l'annexe 2 présente l'intégralité des calculs et des effets sur le bien-être. 


\section{Tableau 1. Les différents cas de l'analyse des mesures non tarifaires}

\begin{tabular}{|c|c|c|}
\hline $\begin{array}{l}\text { Défaillance de } \\
\text { marché }\end{array}$ & $\begin{array}{l}\text { Cadre politique et } \\
\text { réglementaire }\end{array}$ & Principales caractéristiques et issues probables \\
\hline \multirow{11}{*}{$\begin{array}{l}\text { Externalités de } \\
\text { consommation }\end{array}$} & \multirow{3}{*}{$\begin{array}{l}\text { 1) Norme prohibitive ou } \\
\text { interdiction d'importation }\end{array}$} & Pas de concurrence des producteurs étrangers. Autarcie mais qualité élevée. \\
\hline & & Les consommateurs indifférents acquièrent « trop de qualité » \\
\hline & & Le prix payé est élevé, absence d'importations. \\
\hline & \multirow{3}{*}{$\begin{array}{l}\text { 2) Libre-échange (pas } \\
\text { d'étiquetage) }\end{array}$} & Entrée des producteurs étrangers ; l'offre s'élargit, le produit n'est pas différencié \\
\hline & & $\begin{array}{l}\text { Les consommateurs bénéficient d'un prix unique abaissé, les producteurs nationaux sont affectés par la concurrence et par } \\
\text { l'absence d'étiquetage. }\end{array}$ \\
\hline & & Les consommateurs concernés sont affectés par l'imperfection de l'information et par la diminution de la qualité moyenne. \\
\hline & \multirow{5}{*}{$\begin{array}{l}\text { 3) Libre-échange avec } \\
\text { étiquetage obligatoire }\end{array}$} & Les biens et les exigences sont différenciés ; il existe deux prix d'équilibre. \\
\hline & & Chaque type de consommateur peut acheter le produit qu'il préfère. \\
\hline & & Les consommateurs bénéficient de la concurrence de l'offre. \\
\hline & & Grâce à l'étiquetage, les producteurs nationaux voient leurs bénéfices augmenter, avec des prix plus élevés. \\
\hline & & $\begin{array}{l}\text { Problèmes : qui paie l'étiquetage/la certification, dépenses irrécupérables ou coût marginal de l'étiquetage, niveau } \\
\text { protectionniste de l'étiquetage. Risque de parasitisme pour les externalités séparables. }\end{array}$ \\
\hline \multirow[t]{4}{*}{$\begin{array}{l}\text { Externalités de } \\
\text { production }\end{array}$} & \multirow[t]{2}{*}{$\begin{array}{l}\text { 4) Norme prohibitive ou } \\
\text { interdiction d'importation }\end{array}$} & $\begin{array}{l}\text { La norme prohibitive ou l'interdiction d'importation empêchent les importations de produits étrangers qui pourraient } \\
\text { " contaminer » l'offre nationale. Pas d'externalité de production. }\end{array}$ \\
\hline & & La demande est indépendante de l'externalité. \\
\hline & $\begin{array}{l}\text { 5) Libre-échange (pas } \\
\text { d'étiquetage) }\end{array}$ & $\begin{array}{l}\text { L'offre étrangère (importations) « contamine » la production domestique. La courbe de l'offre intérieure pivote en raison de la } \\
\text { hausse des coûts de production induite par l'externalité. L'offre totale répond à la demande pour un prix inférieur. Les } \\
\text { consommateurs profitent d'un prix inférieur à celui du cas d'autarcie. Les producteurs nationaux sont affectés par la baisse des } \\
\text { prix et par la hausse des coûts. }\end{array}$ \\
\hline & $\begin{array}{l}\text { 6) Libre-échange avec } \\
\text { étiquetage obligatoire }\end{array}$ & $\begin{array}{l}\text { Pas étudié ici. Une norme non prohibitive pourrait éliminer l'externalité et accroîtrait le coût unitaire pour les fournisseurs } \\
\text { étrangers. Les producteurs seraient affectés par la concurrence, mais pas par la hausse des coûts. Le prix serait moins élevé } \\
\text { que dans le cas d'autarcie. }\end{array}$ \\
\hline
\end{tabular}




\begin{tabular}{|c|c|c|}
\hline $\begin{array}{l}\text { Défaillance de } \\
\text { marché }\end{array}$ & $\begin{array}{l}\text { Cadre politique et } \\
\text { réglementaire }\end{array}$ & Principales caractéristiques et issues probables \\
\hline \multirow{3}{*}{$\begin{array}{l}\text { Ressources } \\
\text { communes } \\
\text { mondiales, } \\
\text { externalités } \\
\text { séparables et } \\
\text { indivisibles }\end{array}$} & 7) Norme prohibitive & $\begin{array}{l}\text { Conceptuellement similaire au cas où les consommateurs ne disposent pas d'information sur les caractéristiques } \\
\text { négatives. Pas d'effet de retour sur la demande parce que l'externalité est séparable de la consommation. La norme prohibitive } \\
\text { élimine la partie domestique de l'externalité mais pas celle qui a été générée hors du pays. Le bien-être s'améliore avec la } \\
\text { réduction de l'externalité. Le prix payé est relativement élevé. }\end{array}$ \\
\hline & $\begin{array}{l}\text { 8) Libre-échange (pas } \\
\text { d'étiquetage) }\end{array}$ & $\begin{array}{l}\text { Les consommateurs concernés ne sont pas informés du lien entre la consommation et l'externalité. Cette dernière s'accroît. } \\
\text { Pas de retour de l'externalité sur la demande. Le libre-échange abaisse le prix payé mais accroît l'externalité. }\end{array}$ \\
\hline & $\begin{array}{l}\text { 9) Libre-échange et } \\
\text { étiquetage obligatoire }\end{array}$ & $\begin{array}{l}\text { Pas étudié ici. Pas de retour sur la demande, mais réduction de l'externalité séparable, les achats unitaires devant respecter la } \\
\text { norme liée à l'étiquetage. Si l'étiquetage est onéreux à la marge, l'offre pivote à gauche et le prix d'équilibre augmente. Risque } \\
\text { de parasitisme. }\end{array}$ \\
\hline \multicolumn{3}{|l|}{ Cas supplémentaires } \\
\hline $\begin{array}{l}\text { Ressources } \\
\text { collectives } \\
\text { indivisibles }\end{array}$ & $\begin{array}{l}\text { 10) (non analysé dans la } \\
\text { présente étude) }\end{array}$ & $\begin{array}{l}\text { Conceptuellement similaire aux externalités de consommation (cas 1) mais, même avec une politique adaptée et des } \\
\text { consommateurs concernés parfaitement informés, des externalités persistent, l'achat de certains biens par des consommateurs } \\
\text { indifférents ou dans d'autres pays contribuant à l'externalité globale. Risque de parasitisme. }\end{array}$ \\
\hline $\begin{array}{l}\text { Norme non } \\
\text { prohibitive et effort } \\
\text { coûteux }\end{array}$ & 11) & $\begin{array}{l}\text { Les entreprises font des efforts pour réduire les externalités négatives, au prix de dépenses irrécupérables ou d'un coût } \\
\text { marginal de l'effort. La hausse du coût marginal est répercutée sur les consommateurs. Une assez grande partie des dépenses } \\
\text { irrécupérables influe sur le nombre d'entreprises nationales et étrangères (via l'entrée et la sortie). }\end{array}$ \\
\hline
\end{tabular}


Une simplification importante a été effectuée pour que l'exposé reste aussi clair que possible. Dans la discussion ci-après, nous avons pris comme hypothèse initiale que la seule source de défaillance du marché serait étrangère, et que les défaillances de production et de consommation seraient totalement absentes de l'économie intérieure. Cette approche suppose implicitement que les producteurs et les consommateurs nationaux auraient complètement adapté leur comportement de manière à internaliser toutes les externalités qui auraient pu exister auparavant, peut-être en réaction à la réglementation nationale. Cette simplification n'a été adoptée qu'afin que l'exposé reste accessible. Elle est d'ailleurs assouplie par la suite afin d'analyser le cas le plus fréquent selon lequel les producteurs nationaux comme les producteurs étrangers sont soumis à des exigences (normes obligatoires ou volontaires), mais qu'ils doivent fournir des efforts différents pour répondre aux diverses exigences des différents marchés. On peut naturellement imaginer que l'externalité soit d'origine intérieure et soit exportée par le pays. Des externalités positives sont également possibles, comme les transferts de technologie. Ces cas ne sont pas traités dans cette étude, mais pourraient être pris en compte dans le cadre. Le tableau ci-après synthétise les différents cas et met en avant les principales conclusions.

\subsection{Défaillances du marché affectant les consommateurs}

Le bien du marché analysé est supposé homogène (c'est-à-dire présentant les mêmes attributs de qualité), à l'exception d'une caractéristique spécifique qui diffère en fonction du pays d'origine. Nous partons du principe que les producteurs étrangers proposent un bien présentant une caractéristique particulière (risque pour l'environnement ou pour la sécurité, ou processus de production spécifique) rejeté ou moins apprécié par certains consommateurs nationaux, alors que d'autres y sont indifférents. Cette hypothèse rejoint la pratique la plus commune selon laquelle une réglementation est censée protéger certains consommateurs ayant des préférences à l'égard d'une caractéristique des produits étrangers. Il s'agit bien sûr d'une simplification, les producteurs étrangers comme les producteurs nationaux étant soumis à des normes obligatoires dans leur pays d'origine. Cependant, cette simplification à des fins d'analyse permet une focalisation plus précise sur les différentes exigences selon les pays, en mettant en avant la diversité du caractère jugé approprié des caractéristiques des produits. Compte tenu de la diversité de ces exigences, les entreprises nationales et étrangères doivent fournir des degrés d'efforts différents pour répondre aux conditions de production à observer sur les différents marchés.

On suppose initialement que les producteurs étrangers ne sont pas capables de corriger cette caractéristique, ni de réduire l'externalité liée au bien qu'ils proposent. À l'exception de la caractéristique particulière susmentionnée, tous les consommateurs ont les mêmes préférences en termes d'utilité directe liée au produit. La caractérisation des préférences s'inspire largement de Polinsky et Rogerson (1983). Les demandes sont dérivées de préférences quadratiques, et l'offre, d'une fonction de coût quadratique. Pour commencer par les préférences des consommateurs, la demande de chacun d'entre eux, notée $i=\{1, \ldots, N\}$, procède d'une fonction d'utilité quasi-linéaire comprenant les préférences quadratiques pour le bien du marché visé ainsi qu'un numéraire supplémentaire :

$$
U_{i}\left(q_{i}, w_{i}\right)=a q_{i}-\bar{b} q_{i}^{2} / 2-I r_{i} q_{i}+w_{i}
$$


$a q_{i}-\bar{b} q_{i}^{2} / 2$ exprimant la satisfaction immédiate du consommateur $i$ issue de la consommation d'une quantité $q_{i}$ du bien, et $w_{i}$ représentant le bien numéraire consommé par $i$. Pour simplifier, $a, \bar{b}$ sont les mêmes pour les $N$ consommateurs.

Les effets des externalités et de l'information sont exprimés par $-I r_{i} q_{i}$. Le paramètre $I$ représente la connaissance et/ou le contexte d'externalité relatif aux caractéristiques spécifiques apportées par le produit étranger. Si les consommateurs ne sont pas conscients des caractéristiques spécifiques, ou s'il existe une externalité non prise en compte liée à la caractéristique spécifique, alors $I=0$. À l'inverse, $I=1$ signifie que les consommateurs sont conscients de l'existence de la caractéristique spécifique et qu'ils peuvent identifier, sans ambiguïté, le produit étranger, ou bien qu'ils internalisent l'externalité et réduisent leur consommation. Le préjudice perçu associé à la consommation du bien comportant la caractéristique spécifique est noté $-r_{i} q_{i}$.

La maximisation de la fonction d'utilité dans le cadre d'une contrainte budgétaire aboutit à une fonction de demande pour chaque consommateur. La demande totale du bien est obtenue en additionnant les différentes fonctions de demande individuelles pour les $N$ consommateurs. Cependant, la demande totale peut être scindée en deux groupes : les consommateurs indifférents d'une part, et ceux qui se préoccupent d'une caractéristique spécifique du bien (voir l'annexe 2 pour davantage de détails). Mettons qu'une proportion de consommateurs $\beta=N_{l} / N$ soient complètement indifférents à la caractéristique spécifique, avec $r_{i}=0$ pour chaque égalité $i=1, \ldots, N_{l}$. En d'autres termes, ces consommateurs n'attachent pas de valeur de préjudice à la consommation du bien. La proportion de consommateurs concernés restante $(1-\beta)=1-N_{l} / N$ est réticente à consommer la caractéristique spécifique et elle associe au bien un préjudice de consommation égal à $r_{i}=r_{2}$ pour chaque $i=N_{l}+1, \ldots, N^{7}$. Avec $b=\bar{b} / N$, la fonction de demande (inverse) pour les deux sous-groupes devient :

$$
\begin{cases}p_{1}^{D}(Q)=a-(b / \beta) Q & \text { consommateurs indifférents } \\ p_{2}^{D}(Q, I)=a-I r_{2}-[b /(1-\beta)] Q & \text { consommateurs concernés }\end{cases}
$$

Cette spécification est une version mixte associant la différenciation horizontale des produits (à prix identique, les consommateurs choisissent indifféremment un produit ayant la caractéristique et un produit qui en est dépourvu) et la différenciation verticale des produits (à prix identique, les consommateurs concernés choisissent tous le produit dépourvu de la caractéristique négative). En matière de sécurité alimentaire, si tous les consommateurs préfèrent des aliments sains, cette spécification peut être prise en compte grâce à un modèle de différenciation verticale intégrale des produits, où la proportion de consommateurs indifférents est $\beta=0^{8}$.

7. Le cas dans lequel les consommateurs attachent une valeur positive à la caractéristique est complètement symétrique. Il est exprimé en attribuant une valeur négative à $r 2$.

8. Les fonctions de la demande définies par (2) sont linéaires, ce qui représente bien sûr une simplification. Une autre solution consisterait à déterminer la demande non linéaire par 
Du côté de l'offre, on suppose un secteur parfaitement compétitif où les entreprises subissent les prix, aussi bien en ce qui concerne les producteurs locaux que les étrangers. L'ensemble comprend $M_{\mathrm{O}}$ entreprises nationales et $M_{\mathrm{F}}$ entreprises étrangères. Les fonctions de coût des entreprises sont quadratiques pour la production, et c'est cette dernière qui est choisie pour maximiser les profits :

$$
\pi_{s j}=p q_{s j}-1 / 2 c_{s} q_{s j}^{2}-K_{s} \text { for } j=\left\{1, \ldots, M_{s}\right\} ; s=\{O, F\}
$$

avec $c_{s}$ comme paramètre de coût variable et $K_{s}$ comme dépense irrécupérable liée, entre autres, à l'entrée sur le marché de l'entreprise et à sa mise en conformité avec la réglementation. La maximisation du profit aboutit à des fonctions d'offre individuelles pour chaque société, que l'on peut ajouter pour calculer l'offre des entreprises $Q^{9}$ :

$$
\begin{array}{ll}
p_{O}^{S}\left(Q_{O}\right)=c_{O} Q_{O} / M_{O} & \text { offre inverse nationale } \\
p_{F}^{S}\left(Q_{F}\right)=c_{F} Q_{F} / M_{F} & \text { offre inverse étrangère }
\end{array}
$$

L'offre inverse totale est définie comme la somme des offres nationale et étrangère, et notée :

$$
p_{O+F}^{S}\left(Q_{O}\right)=\frac{c_{O} c_{F} Q_{O}}{c_{O} M_{F}+c_{F} M_{O}} \quad \text { offre inverse totale }
$$

Pour le reste de l'analyse, nous supposons que $c_{O}>c_{F}$, ce qui signifie que les producteurs nationaux supportent un coût marginal supérieur à celui des producteurs étrangers. Il s'agit d'une situation dans laquelle la production nationale entraîne un effort coûteux pour éliminer la caractéristique spécifique (risque pour l'environnement/pour la sécurité ou processus de production particulier) rejetée par certains consommateurs, tandis que les producteurs étrangers n'ont pas à supporter ce coût additionnel. Des hypothèses de substitution sont simples à intégrer dans le cadre. Pour simplifier encore davantage, nous supposons initialement que les dépenses irrécouvrables $K_{O}$ et $K_{F}$ sont égales à zéro, ce qui signifie que l'on peut ignorer l'entrée et la sortie de l'entreprise sur le marché. Nous assouplirons cette hypothèse par la suite.

Nous comparons ci-dessous trois configurations de réglementation: (i) une norme prohibitive imposée aux produits étrangers dotés de la caractéristique donnée (équivalente à une interdiction d'importation), (ii) une situation de libre-échange et (iii) une situation de libre-échange prévoyant un étiquetage obligatoire mentionnant la caractéristique négative des produits des entreprises étrangères. Nous n'examinons pas en détail le cas impliquant un étiquetage positif du produit national signalant l'absence de la caractéristique indésirable, mais les résultats seraient techniquement similaires à la situation (iii) dans laquelle seul le produit étranger est étiqueté.

$$
p_{2}=\left[\frac{Q}{\beta\left(1+I r_{2}\right)^{\phi}}\right]^{\frac{1}{\varepsilon}} \text {, où } \phi \text { désigne l'élasticité de l'information et } \varepsilon \text { l'élasticité-prix de la }
$$

demande. Les estimations du bien-être obtenues avec ces deux formules sont proches lorsque l'élasticité-prix de la demande est relativement faible, ce qui est souvent le cas pour les produits alimentaires.

9. Les fonctions d'offre individuelles ne sont définies que pour les prix dépassant les coûts moyens, puisque dans le cas contraire les entreprises cesseraient vraisemblablement de produire le bien. 


\section{Norme prohibitive}

La situation d'autarcie qui découle de l'imposition d'une norme prohibitive est la plus simple à analyser. En effet, dans ce cas, la demande intérieure globale se réduit à une seule fonction, puisque le produit étranger n'est tout simplement pas disponible. Le graphique 1 représente la demande (D) et l'offre $\left(S_{O}\right)$ intérieures. L'axe des ordonnées représente les prix, tandis que la quantité est représentée en abscisse. Avec des importations nulles, il existe un prix d'équilibre du marché unique $p^{A}$ qui égalise la demande et l'offre avec une quantité d'équilibre $Q_{A}$ (de sorte que $\left.p^{D}\left(Q^{A}, 0\right)=p_{O}^{S}\left(Q^{A}\right)\right)$.

Les profits de tous les producteurs nationaux sont contenus dans la zone $O A p^{A} \mathrm{du}$ graphique 1 , tandis que le surplus des consommateurs intérieurs correspond à la zone $A p^{A} a$. Le bien-être national total est égal à la somme des surplus des consommateurs et des producteurs, représentée par la zone $O A a$. Les expressions analytiques complètes des valeurs d'équilibre des prix et des quantités, ainsi que de tous les composants de bienêtre, se trouvent à l'annexe 2 .

\section{Graphique 1. Externalité de consommation, avec norme prohibitive}

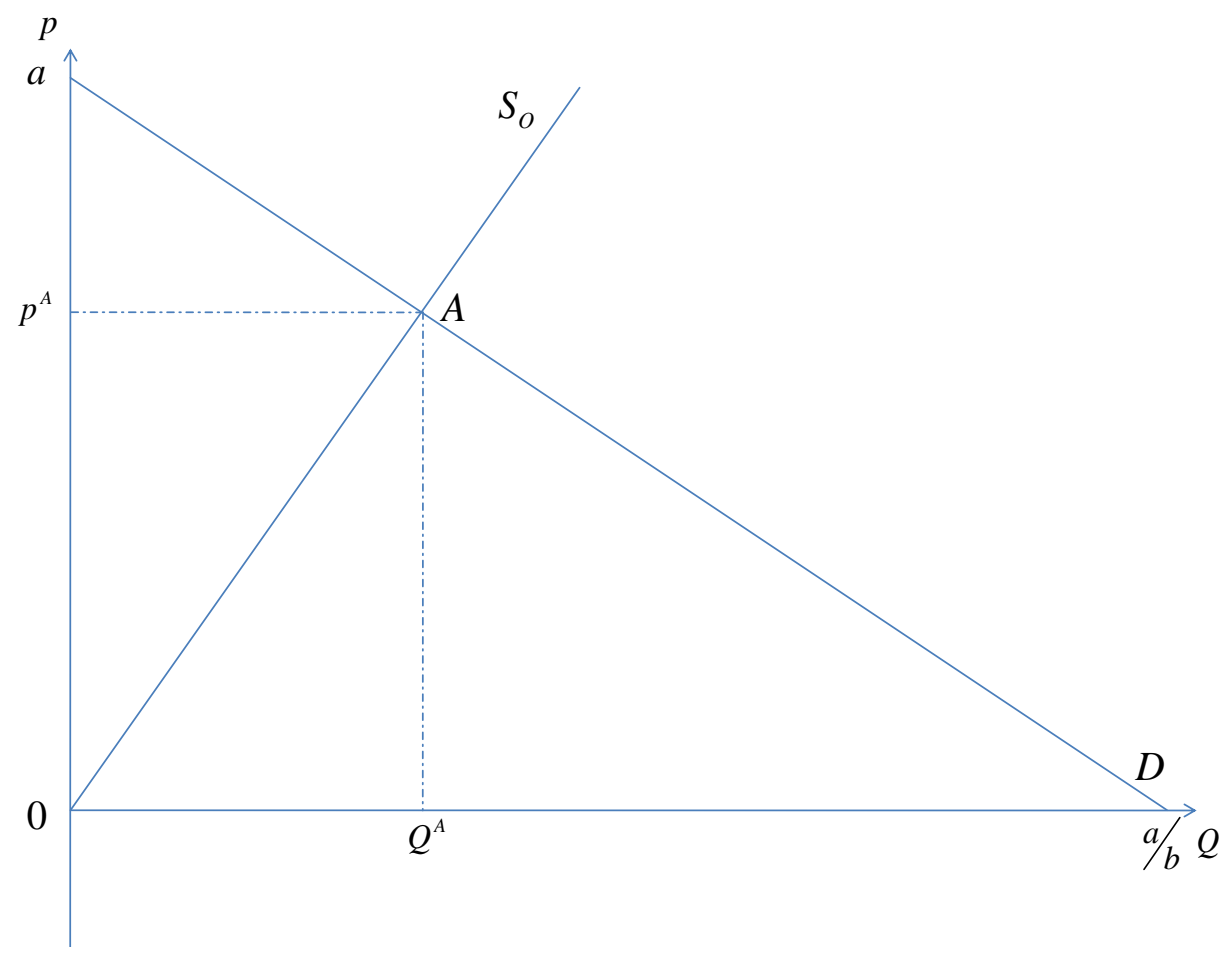

\section{Libre-échange}

L'opposé d'une interdiction d'importation est une situation de libre-échange. Nous l'avons représentée dans le graphique 2, qui comprend également la situation d'autarcie précédente, sous forme de lignes pointillées. L'ouverture des frontières aux produits étrangers (l'offre étant notée $S_{F}$, ) modifie l'allocation du marché. L'offre globale est représentée par $S_{O}+S_{F}$ dans le graphique 2 . 
Du côté de la demande, rappelons qu'il est complètement indifférent à une proportion $\beta$ de consommateurs de choisir entre les produits nationaux et les produits étrangers, puisque ces consommateurs ne sont pas préoccupés par la caractéristique supplémentaire attachée aux seconds. La demande globale de ce sous-groupe est notée $D_{1}$. Une proportion $(1-\beta)$ de consommateurs est préoccupée par les produits étrangers (par exemple, pour des raisons concernant la sécurité, l'environnement, l'éthique ou des questions sociales). Leur propension à payer pour une quantité donnée diminue de $r_{2}$ pour les produits dotés de la caractéristique négative donnée ${ }^{10}$. De ce fait, la demande des consommateurs ayant une préférence devient $\tilde{D}_{2}$ (définie comme $p_{2}^{D}(Q, 1)$ ).

\section{Graphique 2. Externalité de consommation, situation de libre-échange}

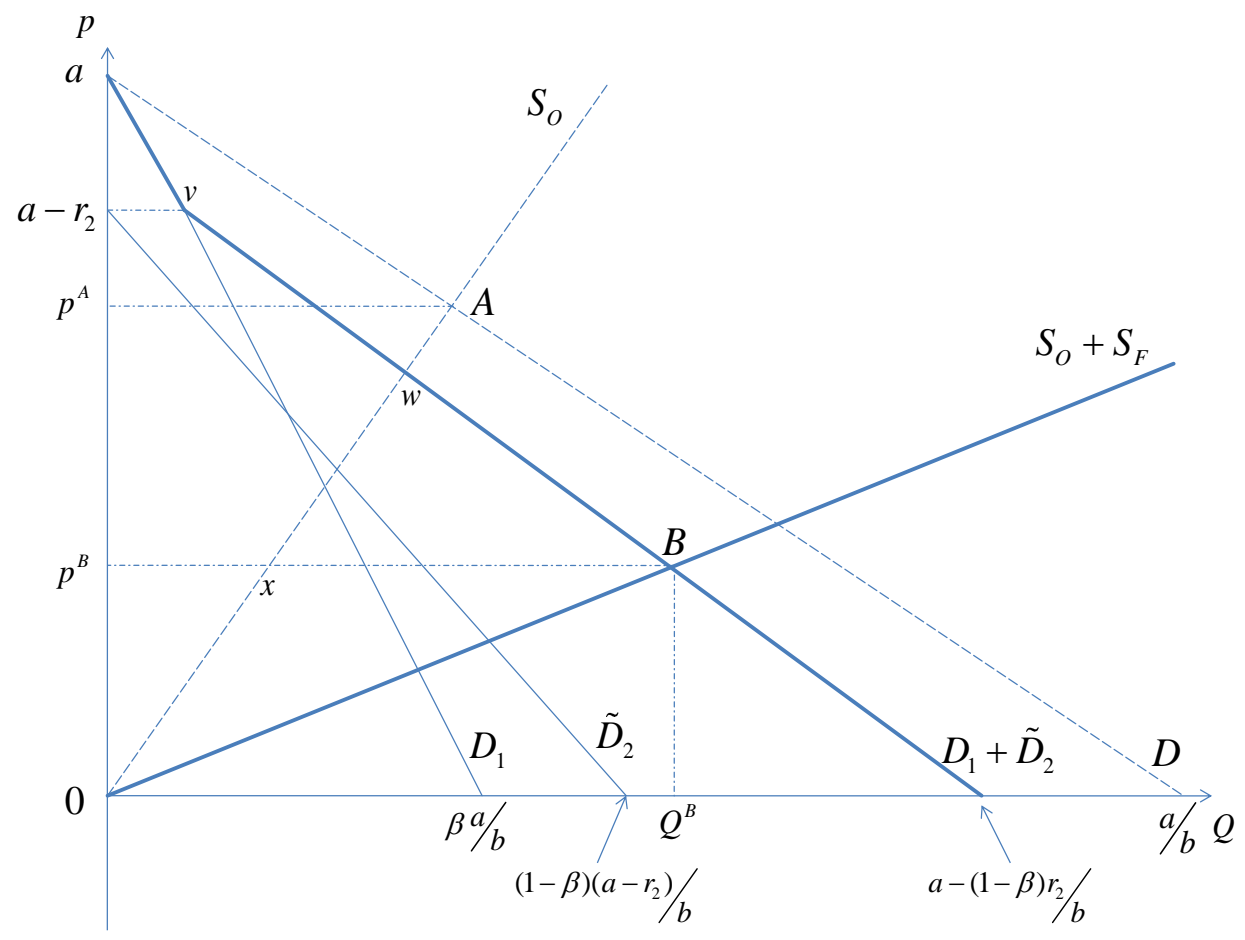

Pour cette configuration de libre-échange, sans étiquetage, la demande globale est représentée par $D_{1}+\tilde{D}_{2}$ dans le graphique 2. Le préjudice unitaire $r_{2}$ implique une cassure du tracé de la demande globale au point $v$ de ce même graphique. Dans un tel état des choses, il n'existe qu'un prix d'équilibre du marché, puisque l'absence d'information sur la caractéristique du produit interdit de faire une différence entre les deux qualités et de

10. Nous avons simplifié la demande des consommateurs, en faisant abstraction des stratégies de recherche ou d'expérience, ainsi que des mentions de qualité/sécurité (par le biais d'un investissement dans une marque ou d'une garantie) et/ou de la réputation d'une entreprise dans un contexte d'achats répétés avec une situation d'information imparfaite. Les attentes rationnelles de qualité supposent de la part des consommateurs une connaissance parfaite de tous les paramètres (connaissance commune) des modèles d'information. Une telle exigence n'est absolument pas réaliste puisqu'une certaine expertise technique est nécessaire à la connaissance de certains attributs. 
segmenter l'offre. L'équilibre du marché entraîne un prix d'équilibre $p^{B}$ tel que la demande est égale à l'offre, et détermine une quantité d'équilibre $Q^{B}$.

Les profits des producteurs nationaux correspondent à la zone $O x p^{B}$ du graphique 2. On voit clairement que les entreprises nationales dégagent des bénéfices moindres que dans la situation d'autarcie ; $O x p^{B}<O A p^{A}$. Le surplus des consommateurs intérieurs correspond à la zone $p^{B} B v a$, tandis que le bien-être national total est représenté par la zone $O x B v a$. Le profit du producteur étranger est noté $O x B$. Ce dernier bénéficie clairement de la libéralisation des échanges par rapport à la situation d'autarcie, dans laquelle son profit était égal à zéro.

Le graphique 2 illustre également la variation du bien-être national au moment du passage de l'autarcie au libre-échange. On peut identifier ici deux effets opposés. Le premier procède du caractère imparfait de l'information pour la proportion $(1-\beta)$ de consommateurs concernés, réticents à acquérir les produits étrangers. Comme ces consommateurs ne peuvent opérer de différenciation entre les deux types de biens, ils réduisent leur demande à un prix donné et leur surplus diminue. Le second effet correspond à l'effet de prix décroissant issu de l'augmentation de l'offre liée à la pénétration du produit étranger sur le marché.

La conséquence de la libéralisation des échanges dans le pays importateur, à savoir la comparaison entre le bien-être $O A a$ en situation d'autarcie et le bien-être $O x B v a$ en situation de libre-échange, est ambiguë. Si la zone $x B w$ est plus étendue que la zone $v a A w$, alors la diminution de prix est suffisamment importante pour que la libéralisation des échanges profite au pays importateur. En revanche, il peut arriver que la zone $x B w$ soit moins étendue que la zone $v a A w$, lorsque la libéralisation des échanges suppose une baisse relativement marquée de la demande de la proportion (1- $\beta$ ) de consommateurs intérieurs avertis. Dans ce second cas, la libéralisation des échanges entraîne une perte de bien-être pour le pays importateur, et il serait alors préférable, du point de vue national, de mettre en place une interdiction de commerce liée à une norme servant d'entrave aux produits étrangers présentant la caractéristique spécifique. Les producteurs étrangers seraient affectés par une telle décision, et subiraient une perte égale à $O x B$.

\section{Libre-échange avec étiquetage obligatoire}

L'option du libre-échange peut entraîner une diminution du bien-être national si la proportion de consommateurs concernés est suffisamment importante et si ceux-ci, en raison du caractère imparfait de l'information sur les caractéristiques du bien, réduisent suffisamment leur demande pour occulter les bénéfices tirés du prix inférieur des importations. Notre troisième configuration associe le libre-échange à un étiquetage obligatoire qui mentionne parfaitement la caractéristique négative attachée au produit étranger. Pour simplifier, cet étiquetage est supposé compris par tous les consommateurs et véhicule la totalité des informations pertinentes à ces derniers ${ }^{11}$. Pour simplifier encore davantage, nous supposons ici que l'étiquetage ne coûte rien, une hypothèse que l'on pourra aisément assouplir. L'étiquetage permet de segmenter le marché en deux, avec, d'un côté, une offre de produits étrangers qui comprend la caractéristique rejetée par

11. Wansink et al (2004) analysent les limites de l'étiquetage en matière de communication d'information. Certaines caractéristiques de qualité précisées sur l'étiquette pourront être difficilement compréhensibles pour les consommateurs, tandis que certains étiquetages pourront dérouter l'acheteur et affecter la crédibilité des étiquettes mieux conçues. 
certains consommateurs, et, de l'autre, une offre de produits nationaux, qui ne présente pas cette caractéristique. On suppose que la segmentation est parfaite et qu'il ne peut exister aucune vente d'arbitrage entre les deux segments. L'offre étrangère $S_{F}$ est à présent représentée dans le graphique 3, la situation précédente de libre-échange apparaissant maintenant sous forme de lignes pointillées (avec la situation d'équilibre précédente au point $\mathrm{B})$.

\section{Graphique 3. Externalité de consommation, situation d'étiquetage obligatoire}

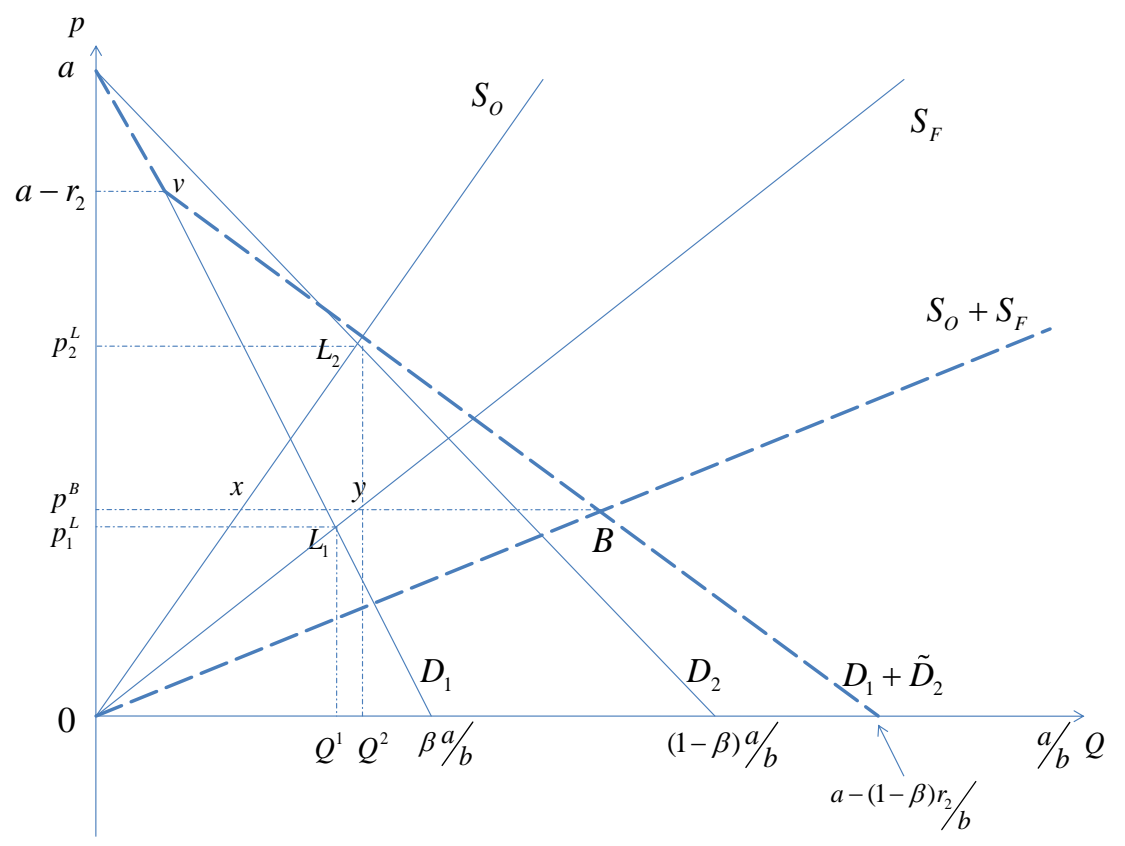

Dans la situation d'étiquetage, il existe deux prix d'équilibre du marché, puisque l'étiquetage permet d'identifier les deux qualités en segmentant l'offre. La proportion (1$\beta$ ) de consommateurs concernés peut à présent se tourner vers le produit d'origine nationale qui ne présente pas la caractéristique négative, de sorte que la demande $D_{2}$ reste identique à celle de la situation d'autarcie, avec $p_{2}^{D}(Q, 0)$.

Le premier prix d'équilibre, $p_{1}^{L}$, suppose l'égalité entre $p_{1}^{D}(Q)$ et $p_{F}^{S}(Q)$, lorsque les produits étrangers sont achetés par la proportion $\beta$ de consommateurs indifférents qui recherchent le prix le plus bas. Le surplus des consommateurs indifférents est noté $p_{1}^{L} L_{1} a$ et les profits des producteurs étrangers sont représentés par $0 p_{1}^{L} L_{1}$. Le deuxième prix d'équilibre, $p_{2}^{L}$, suppose l'égalité entre $p_{2}^{D}(Q, 0)$ et $p_{O}^{S}(Q)$, lorsque les produits nationaux sont achetés par la proportion $(1-\beta)$ de consommateurs concernés qui évitent les produits étrangers. Le surplus des consommateurs concernés est égal à $p_{2}^{L} L_{2} a$ et les profits des producteurs nationaux sont notés $0 p_{2}^{L} L_{2}$. Les producteurs nationaux voient leurs bénéfices augmenter par rapport à la situation de libre-échange sans étiquetage (ce bénéfice était alors noté $0 p^{B} x$ ).

Le bien-être national correspond à la zone $p_{1}^{L} L_{1} a+0 L_{2} a$ dans le graphique 3. Dans ce troisième scénario de politique économique, le bien-être est supérieur à celui obtenu 
dans le cadre du libre-échange sans étiquetage mesuré par OxBva. La politique d'étiquetage permet aux entreprises nationales de réaliser des bénéfices supérieurs et autorise une plus grande diversité de l'offre pour les consommateurs, qui contribue à l'amélioration du bien-être, par comparaison avec la situation de libre-échange sans étiquetage. Du point de vue des consommateurs, cette situation est la plus souhaitable, puisque l'étiquetage fournit une information et que la libéralisation des échanges contribue à faire baisser les prix.

Dans la situation d'étiquetage, le profit des producteurs étrangers est noté $0 p_{1}^{L} L_{1}$, ce qui reste nettement inférieur au bien-être $0 p^{B} y$ obtenu dans la situation de libre-échange sans obligation d'étiquetage. Imposer une obligation d'étiquetage peut faire l'objet d'une controverse entre les deux pays concernés, puisque cela supposerait une érosion des bénéfices des producteurs étrangers.

Les conclusions sur le plan du bien-être qui découlent de l'analyse ci-dessus dépendent clairement de la configuration des paramètres. Les élasticités de la demande et l'ampleur de l'évaluation de l'externalité par le consommateur $\left(r_{2}\right)$ déterminent l'évaluation finale ; or, ces paramètres devront être déterminés d'une manière empirique. De même, s'il s'avère que les entreprises nationales supportent un coût marginal inférieur à celui des fournisseurs étrangers, ce qui les autorise à proposer leurs produits à un prix inférieur à celui des produits importés, alors même les consommateurs non concernés seront tentés d'acheter les biens produits dans leur pays. De telles différences de coûts peuvent également résulter de la politique économique elle-même.

Il est clair que la mise en application de l'obligation d'étiquetage entraîne des coûts privés et publics liés à la certification des produits et des unités de production, à la préservation de l'identité et à la promotion de l'étiquetage (voir Bureau et al., 1998). L'étiquetage accroît également les coûts de production, puisqu'il suppose une modification des processus de fabrication et d'emballage (pour des exemples des coûts et avantages de l'indication du pays d'origine sur les conditionnements de jus de fruits, voir Centre for International Economics (2006)). Si ces coûts ne sont pas «trop » élevés (c'est-à-dire non prohibitifs), l'État jugera que l'obligation d'étiquetage représente une solution optimale.

L'incidence des coûts d'étiquetage représente un facteur de complication. La répartition optimale des coûts entre les consommateurs, les contribuables, les producteurs et l'État dépend de la nature des coûts d'inspection et de certification (voir Crespi et Marette, 2001). Si les entreprises nationales et étrangères supportent les mêmes coûts d'inspection et de certification qui dépendent des quantités produites, la théorie économique suggère qu'ils sont pris en compte dans le prix facturé au consommateur. Dans ce cas, les deux courbes d'offre $S_{O}$ et $S_{F}$ se déplacent parallèlement vers le haut dans le graphique 3. À l'inverse, si les entreprises supportent des coûts irrécouvrables (sunk cost) d'inspection et de certification (i.e. occasionnés en pure perte), alors ils ne seront pas directement intégrés au prix final demandé au consommateur. Certaines sociétés pourront se retirer du marché jusqu'à ce que les entreprises restantes atteignent le point d'équilibre avec un prix du marché plus élevé au nouveau point d'équilibre. Dans ce cas, les deux courbes d'offre $S_{O}$ et $S_{F}$ pivotent vers le haut dans le graphique 3 et l'analyse est censée suivre le nombre respectif d'entreprises nationales et étrangères. Quel que soit le type de coût, plus la certification et l'inspection seront onéreuses, moins l'imposition d'un étiquetage obligatoire sera avantageuse. 
Le risque de controverse entre les deux pays concernés au moment de l'examen des politiques économiques applicables est exacerbé lorsque les coûts d'investissement sont supportés par les producteurs étrangers. En effet, comme ces coûts irrécouvrables ne sont pas répercutés sur le consommateur directement dans le prix de vente, il est optimal pour un État de transférer aux producteurs étrangers les dépenses irrécouvrables liées à l'étiquetage. Un coût prohibitif peut décourager les producteurs étrangers de pénétrer le marché national, ce qui en fait une barrière à l'entrée.

La politique d'étiquetage analysée dans cet exemple ne précise pas le type de mention à apporter. Des informations sur les caractéristiques du produit peuvent prendre la forme d'un étiquetage «négatif» indiquant les caractéristiques du produit non désirées par certains consommateurs. Au contraire, un étiquetage «positif» mettrait en valeur de façon constructive le respect d'une certaine norme de production. Pour la logique de notre analyse, cette différence n'a pas d'importance tant que le consommateur est capable d'opérer une distinction totale entre les deux types de produits. Cependant, en termes d'application d'une politique d'étiquetage, il peut exister d'importantes différences entre les deux approches. Les consommateurs pourront réagir différemment à une mention négative et à un signe positif.

\subsection{Défaillances du marché affectant les producteurs}

Les défaillances liées à la production, telles que les maladies affectant les animaux ou les plantes, peuvent être conceptualisées comme un choc négatif sur l'offre, comme on le voit dans le graphique 4, ce qui entraîne un pivot vers le haut de la courbe du coût marginal, dans la lignée d'Orden et Romano (1996), de Wilson et Anton (2006) et de Peterson et Orden (2008). L'offre initiale $S_{O}$ en situation d'autarcie est représentée par des lignes pointillées sur le graphique 4. La demande des consommateurs $D$ est supposée inchangée par le régime commercial.

Avec l'importation de produits étrangers, l'externalité est transférée au marché intérieur et affecte la demande nationale, avec un pivot de $S_{O}$ vers $\bar{S}_{O}$. Cette modification de l'offre nationale suite à l'ouverture du marché face à une perte potentielle de production liée à la survenance d'une maladie peut être représentée d'une manière assez simple. Soit $\lambda$ la probabilité de perte de production suite à la survenance de l'externalité. En supposant une neutralité des producteurs nationaux face au risque, la maximisation des profits individuels suppose d'opter pour une production de manière à maximiser $\bar{\pi}_{O j}=p(1-\lambda) q_{O j}-1 / 2 c_{O} q_{O j}^{2} . \bar{\pi}_{O j}$ correspond au profit attendu avant la réalisation de la perte, puisque la décision de production est prise avant le moment probable de cette dernière. La résolution du problème de maximisation individuel et l'addition de tous les producteurs aboutit à l'offre nationale totale : $\bar{S}_{O}(p)=\sum_{j=1}^{m} \bar{q}_{O j}=m(1-\lambda) p / c_{O}$. Plus $\lambda$ sera élevé, plus le pivot de la courbe inverse de l'offre $\bar{S}_{O}$ par rapport à l'axe des ordonnées du graphique 4 sera important ${ }^{12}$.

12. Un prolongement du cadre pourrait envisager le cas dans lequel certaines externalités influent sur l'offre étrangère $S_{F}$. 
Dans des conditions de libre-échange, le prix d'équilibre attendu est $p^{G}$ et le bien-être national total attendu est représenté par la zone $0 \gamma G a$. La comparaison du bien-être en situation d'autarcie et de libre-échange consiste à comparer les zones $0 \gamma \mu$ et $\mu G A$ (rappelons que, dans la situation d'autarcie, le bien-être correspond à $O A a$ ). L'effet de bien-être est ambigu a priori. Si la zone $\mu G A$ est plus étendue que la zone $0 \gamma \mu$, alors le bien-être national s'accroît avec la situation de libre-échange par rapport au régime économique faisant obstacle aux importations. Ce cas correspond à une situation comportant une faible probabilité $\lambda$ de perte de production liée à la libéralisation des échanges.

Graphique 4. Externalité de production, situation de libre-échange

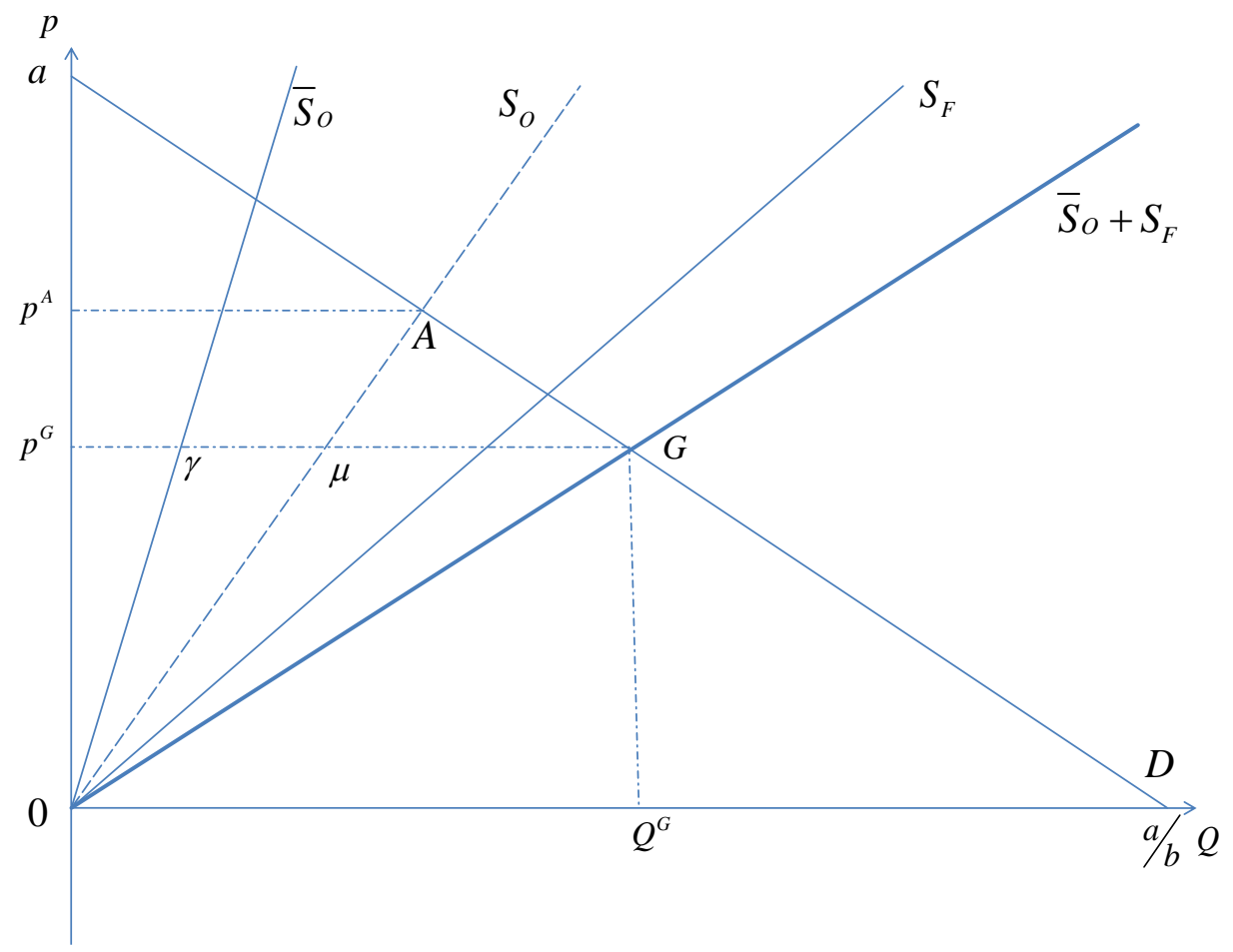

Notons que l'on peut introduire une approche dynamique en tenant compte des probabilités variables en fonction du moment. On peut intégrer des flux sur plusieurs périodes au moyen d'un facteur d'actualisation appliqué aux mesures de bien-être présentées dans le graphique 4. Il est possible d'enrichir encore le modèle, mais au final la probabilité de l'externalité progresse avec les échanges.

\subsection{Externalité de ressources collectives mondiales}

Deux cas peuvent être distingués dans un contexte d'externalité de ressources collectives mondiales. Comme nous le verrons, les décisions de consommation du marché et l'externalité négative sont séparables dans les deux cas.

Dans un premier cas simple, les consommateurs subissent une perte de bien-être issue de l'externalité, indépendamment de leur décision de consommation. Par exemple, la biodiversité est compromise même si la décision de consommation ne contribue pas 
directement à cette défaillance. La perte issue de cette externalité indivisible est séparable de l'utilité dérivée de la consommation et n'influe pas sur la demande d'un bien. Même si cela peut sembler paradoxal, cette situation est conceptuellement équivalente à celle dans laquelle les consommateurs concernés n'intègrent pas la caractéristique négative supplémentaire dans leur demande (avec $I=0$ dans l'équation 1 ). Le concept est similaire à celui de l'analyse des externalités de consommation, mais avec une différence de taille : les consommateurs concernés ne peuvent internaliser totalement l'externalité via leurs décisions de consommation individuelles. Il convient de prendre en compte l'externalité dans les calculs du bien-être, mais celle-ci ne se reflète pas dans le schéma de demande.

Dans le graphique 5, le libre-échange aboutit à un prix d'équilibre $p^{E}$ qui marque l'égalité entre la demande et l'offre totales (nationales et étrangères). Comme leur décision de consommation ne contribue nullement à réduire l'externalité globale, elle n'a aucun impact sur la demande des consommateurs concernés lorsque le marché s'ouvre aux producteurs étrangers (dans l'équation (2) la demande correspond à $p_{2}(Q, 0)$ ). Cependant, il conviendrait de tenir compte de l'externalité dans le calcul du bien-être, mais le moyen de mesurer exactement celle-ci n'est pas parfaitement clair. Une approche consiste à prendre les quantités importées et consommées, puis de les multiplier par le préjudice unitaire que les consommateurs concernés associent à l'externalité des ressources communes. Il s'ensuivrait une valeur de l'externalité $r_{2}(1-\beta) Q^{E}=r_{2} Q_{2}^{E}$, avec $Q_{2}^{E}$ représentant la consommation par la proportion $(1-\beta)$ de consommateurs concernés au prix $p^{E}$. Cette approche consistant à intégrer les externalités de ressources communes mondiales comme des entités séparables de la consommation s'inspire de Foster et Just, 1989, et de Teislet al, 2001. L'ajout des surplus habituels des consommateurs (zone $p^{E} E a$ ) et des producteurs (zone $O z p^{E}$ ) à l'externalité (zone $\left.0 r_{2} t Q_{2}^{E}\right)$ aboutit au bien-être national global dans des conditions de libre-échange sous la forme de la zone $0 z P^{E}+P^{E} E a-0 r_{2} t Q_{2}^{E}$.

L'imposition d'une interdiction d'importation supprime l'offre étrangère du marché national. La comparaison de bien-être entre les situations d'autarcie et de libre-échange consiste à comparer les zones $0 r_{2} t Q_{2}^{E}$ et $z E A$ (le surplus combiné des consommateurs et des producteurs en autarcie aboutit à un bien-être total représenté par la zone OAa ). La zone $z E A$ représente un gain de bien-être dans la situation de libre-échange, tandis que la zone $0 r_{2} t Q_{2}^{E}$ correspond à la valeur de la perte associée par les consommateurs concernés à l'externalité globale négative. Le bien-être en situation d'interdiction d'importation s'accroît si le préjudice unitaire $r_{2}$ est suffisamment important pour compenser les avantages d'une baisse des prix intérieurs dans un régime de libre-échange.

L'analyse ci-dessus a pris comme base une évaluation des quantités de biens importés et consommés liée à l'externalité des ressources communes mondiales. Il est également possible, si l'externalité provient de la production (comme tel est le cas pour la destruction de la forêt tropicale), de mesurer sa taille à partir de la quantité totale de production étrangère, et non seulement de celle importée et consommée sur le marché intérieur. Cette approche aboutirait à une valeur de l'externalité dépendant de la production étrangère totale, et la valeur augmenterait parallèlement à la production totale (plus on détruit de forêt, plus la valeur accordée à la surface restante augmente). Bien que cet autre mode de mesure de l'externalité ne se prête pas aisément à la représentation dans 
le graphique 5, son principe n'est pas fondamentalement différent de la comptabilisation du bien-être à partir des importations. Comme dans le cas précédent, les surplus des consommateurs et des producteurs devront intégrer une évaluation de l'externalité de ressources communes mondiales pour mesurer le bien-être total dans un autre cadre de politique économique.

Un second cas, que nous ne développons pas entièrement ici, comprend les ressources collectives mondiales lorsque les consommateurs concernés réduisent leur consommation pour se sentir mieux, malgré un impact individuel réduit sur l'externalité indivisible. Dans ce cas, l'externalité se reflète dans les décisions de demande des consommateurs concernés mais l'externalité ne peut, en règle générale, être entièrement internalisée. La diminution de consommation est trop marginale pour inciter les fournisseurs, dont l'offre est à l'origine de l'effet négatif sur les ressources collectives, à changer de comportement. Pour se sentir mieux, les consommateurs concernés réduiront leurs achats de biens liés au problème des ressources communes mondiales. Ils pourront éventuellement arbitrer en faveur d'un bien issu du développement durable, étiqueté écologique, si celui-ci est disponible. Ce dernier cas correspond à celui dans lequel les consommateurs concernés sont informés $(I=1)$ dans l'équation (2), alors même que ces derniers n'internalisent pas totalement l'externalité, puisque l'effet regrettable sur les ressources collectives persiste. Ce deuxième cas associe des éléments du cas dans lequel les consommateurs concernés ajustent leur propre consommation à cause de l'externalité négative ou arbitrent en faveur d'un substitut issu du développement durable, qui répond aux exigences de l'étiquetage écologique. Cependant, l'externalité n'est pas totalement internalisée du fait que d'autres consommateurs, sans préférences, achètent le bien ordinaire qui contribue au problème affectant les ressources collectives mondiales. L'externalité peut être réduite, mais pas éliminée totalement, à moins que tous les consommateurs dans le monde entier n'achètent que les biens étiquetés écologiques.

\section{Graphique 5. Externalité des ressources communes mondiales}

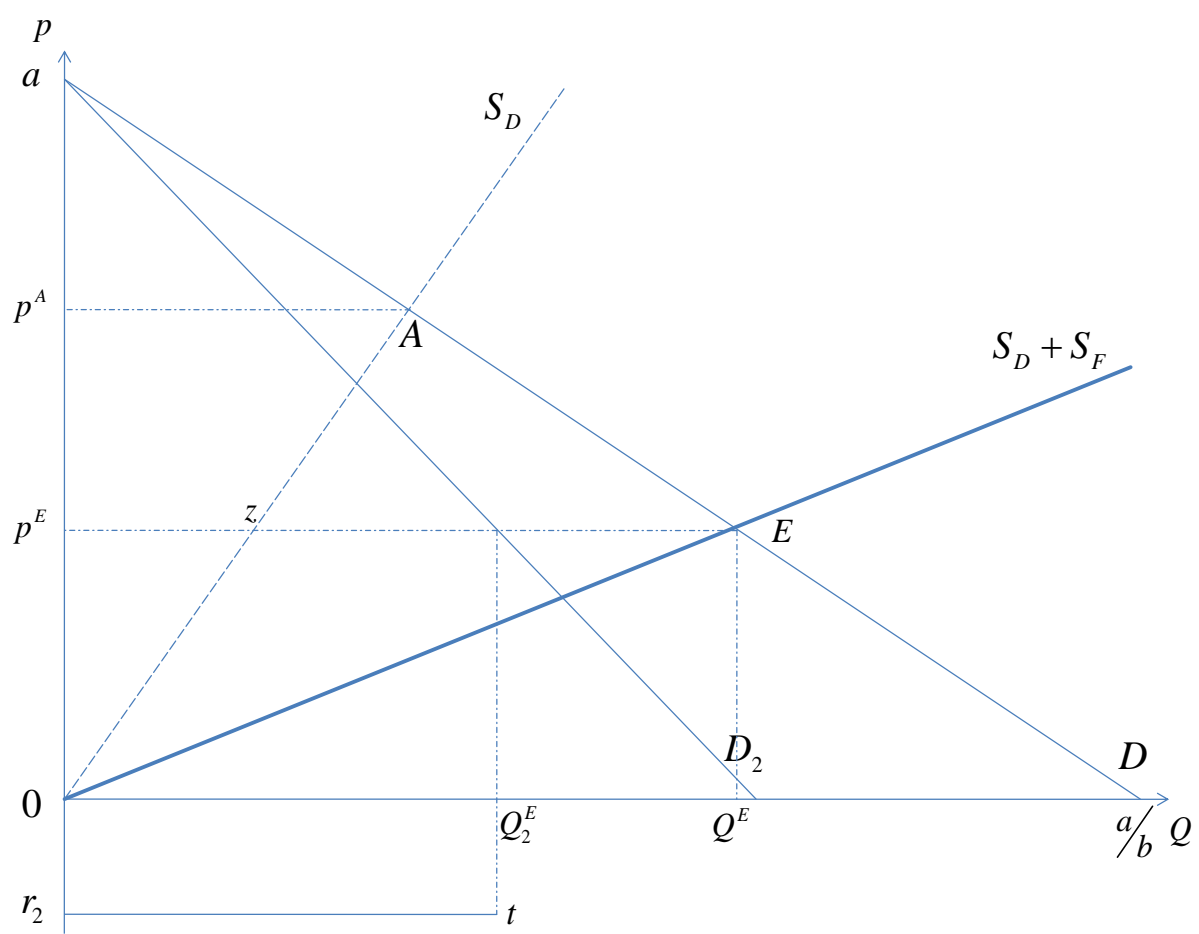




\subsection{Développements}

\section{Norme non prohibitive}

Lors de la présentation précédente du cadre coûts-avantages, la norme était supposée prohibitive, et donc assimilable à une interdiction d'importation. Dans une approche plus réaliste, la norme toucherait à la fois l'offre nationale et l'offre étrangère, accroissant ainsi les coûts de production et réduisant l'impact de la caractéristique spécifique que le consommateur concerné souhaite éviter. Pour introduire une norme non prohibitive, il convient de reformuler comme suit l'utilité des consommateurs et les profits des producteurs :

$$
\begin{aligned}
& U_{i}\left(q_{i}, w_{i}\right)=a q_{i}-\bar{b} q_{i}^{2} / 2-I \times(1-\rho) \times r_{i} q_{i}+w_{i}, \\
& \pi_{O j}=p q_{O j}-1 / 2 c_{O} q_{O j}^{2}-k_{O}(\rho) q_{O j}-K_{O}(\rho), \text { et } \\
& \pi_{F j}=p q_{F j}-1 / 2 c_{F} q_{F j}^{2}-k_{F}(\rho) q_{F j}-K_{F}(\rho) .
\end{aligned}
$$

Les entreprises fournissent un effort $\rho$, tel que $0 \leq \rho \leq 1$, qui réduit le préjudice $r_{i} q_{i}$ pour les consommateurs. Dans ce cadre, cet effort augmente les coûts marginaux des entreprises nationales et étrangères $k_{O}(\rho)$ et $k_{F}(\rho)$, de même que les dépenses irrécouvrables (sunk cost) $K_{O}(\rho)$ et $K_{F}(\rho)$. Les entreprises doivent à présent prendre deux décisions interdépendantes, concernant le niveau d'effort à fournir pour respecter la norme, et le niveau de production. Le choix optimal dépendra de la structure du marché et du type d'interaction stratégique entre les entreprises. Dans une situation d'équilibre, les coûts marginaux de l'effort sont répercutés sur les consommateurs à travers le prix de vente. Lorsque les entreprises doivent supporter des coûts irrécouvrables de mise en conformité, alors ceux-ci ne sont pas directement transférés au prix final.

Pour procéder à l'analyse coûts-avantages d'une norme, on peut partir d'un niveau donné ou bien déterminer un niveau optimal de la norme en laissant les instances de réglementation nationales ou internationales tenir compte d'une mesure du bien-être (Fisher et Serra, 2000). Par exemple, une autorité de réglementation nationale pourra choisir une norme qui maximise le bien-être national (défini comme la somme des surplus des agents domestiques). Ce niveau pourra affecter les producteurs étrangers s'il entraîne des coûts prohibitifs, $k_{F}(\rho)$ et $K_{F}(\rho)$. D'un autre côté, une autorité de réglementation internationale choisirait une norme qui maximiserait le bien-être mondial. Enfin, l'on peut également envisager une combinaison de plusieurs instruments, tels qu'une norme et un étiquetage (voir Marette (2007) et (2008)).

\section{Considérations relatives à l'organisation industrielle, entrée et sortie des entreprises sur le marché}

On a supposé dans un premier temps que les dépenses irrécouvrables $K_{O}$ et $K_{F}$ étaient nulles. Cette hypothèse permet d'ignorer la sortie du marché d'une entreprise. Cependant, si les dépenses irrécouvrables sont supérieures à zéro, alors les bénéfices peuvent passer en territoire négatif, ce qui incitera certaines entreprises à se retirer du marché. Si les dépenses irrécouvrables dépendent des efforts de mise en conformité, alors 
une norme peut avoir pour conséquence le retrait de certaines entreprises. Certaines sociétés étrangères pourront quitter le marché jusqu'à ce que les autres fournisseurs de produits atteignent le point d'équilibre. Ce point est particulièrement sensible si les coûts fixes de respect de la norme sont plus élevés pour les entreprises étrangères que pour les sociétés nationales. Rau et van Tongeren (2007) montrent l'impact de coûts de mise en conformité fixes et variables sur la structure du marché, à travers l'exemple de fournisseurs polonais respectant et ne respectant pas les normes UE de sécurité dans le domaine de la viande.

Par le biais d'une analyse novatrice, Sutton (1991) montre que les activités ayant un effet de modification de la demande, telles que les normes, peuvent également être employées par une entreprise ou par une autorité de réglementation contre la concurrence, et en particulier contre les sociétés étrangères. Ces travaux nous indiquent notamment que la concentration augmente parallèlement à la taille du marché, par exemple via la libéralisation des échanges, si l'attribut modifiant la demande est produit à un coût irrécouvrable endogène, comme on l'a vu plus haut. Bien que l'analyse de Sutton s'intéresse à la recherche et développement et au marketing, ses conclusions s'appliquent aussi bien aux normes de sécurité ou de qualité alimentaire. En optant pour un niveau de norme de qualité et de sécurité relativement élevé, synonyme de coûts de démarrage importants, les concurrents étrangers potentiels peuvent être éconduits du marché national. Étant donné que les acteurs historiques du marché ne répercutent pas leurs dépenses irrécouvrables sur le client final, ils peuvent adopter une stratégie tarifaire agressive, éliminant ainsi leurs concurrents potentiels. En conséquence, la concentration des producteurs augmente et la variété des produits est susceptible de diminuer. Ce dernier mécanisme se retrouve également dans les modèles commerciaux comprenant des entreprises hétérogènes. Chaney (2005), ainsi que Rau et van Tongeren (2008), montrent qu'une augmentation des coûts fixes d'exportation se traduit par une diminution de la marge commerciale extensive (moins de variétés) en raison du retrait du marché d'exportateurs moins productifs. Au contraire, une hausse des coûts commerciaux variables entraîne une réduction de la marge extensive mais aussi de la marge commerciale intensive (moins d'exportations par tous les acteurs historiques du marché).

\section{Filières et relations verticales}

Le cadre mis en place ici s'est concentré sur le produit final, ignorant les effets des mesures non tarifaires sur les activités d'amont et d'aval. Nous pourrions les inclure en modélisant les étapes successives de la transformation. Il serait notamment possible d'introduire un marché pour les facteurs de production, ce qui rendrait endogènes les coûts variables $c_{O}$ et $c_{F}$. On pourrait ainsi mesurer l'impact relatif d'une norme imposée aux facteurs de production plutôt qu'aux biens produits.

La mise en place de relations verticales entre les fournisseurs de la chaîne de l'offre soulève la question des normes privées. Reardon et al. (2003), Fulponi (2006), OCDE (2006 a,b) et OCDE (2008 a,b), soulignent l'importance croissante de ces dernières. Leur développement a des implications majeures en termes de transfert de la responsabilité des agences publiques vers les entreprises privées. On peut ainsi se demander dans quelle mesure la réglementation dépend plutôt de considérations privées que publiques. Le développement récent de la réglementation privée accroît également l'éventail de normes auxquelles les entreprises doivent se conformer, ce qui est susceptible d'augmenter de manière significative la charge réglementaire totale sur le système alimentaire et sur les consommateurs. 


\section{Consommateurs et gouvernements étrangers}

Une analyse complète doit englober les consommateurs et les gouvernements étrangers, qui imposent peut-être leurs propres politiques. Étant donné que les réglementations tendent à différer d'un pays à l'autre, surtout en cas d'écarts de développement, ce point soulève la question de la reconnaissance mutuelle et de l'harmonisation.

\section{Contrôles et autres mesures non tarifaires aux frontières}

Les contrôles aux frontières et autres mesures non tarifaires aux douanes, telles que la mise en place d'un point d'entrée unique, peuvent entraîner une augmentation des coûts commerciaux variables. Ceux-ci peuvent être aisément incorporés sur le plan conceptuel via la mise en place d'un équivalent-tarifaire (par rapport à ces mesures), qui accroissent le prix payé par le consommateur intérieur pour le bien provenant de l'étranger. Hummels (2001) a créé une méthode permettant d'estimer de manière empirique l'équivalent tarifaire des retards aux frontières.

\section{Contrôles préalables à la livraison}

Au lieu de contrôler les biens à leur arrivée à destination, il est possible de vérifier leur conformité au point de départ (voire sur le lieu de production). Pour évaluer l'effet de ces mesures, il convient de tenir compte de l'agent qui supporte les coûts du contrôle préalable à la livraison. Si c'est le pays de destination, alors ces coûts doivent être comptabilisés comme des dépenses pour le pays importateur. En revanche, s'ils sont supportés par l'exportateur, ils viennent logiquement alourdir les frais d'exportation. Les coûts de contrôle peuvent également être répartis entre les deux pays. Quel que soit l'acteur du marché redevable du coût du contrôle, son incidence joue le rôle d'une taxe sur les importations.

\section{Avancées récentes en matière d'évaluation des défaillances du marché et des ressources collectives mondiales}

Cette partie s'intéresse aux avancées récentes concernant les mesures empiriques de la valeur accordée par les consommateurs pour les caractéristiques des produits. On peut considérer cette partie comme la plus critique de notre analyse, puisque la propension des consommateurs à payer pour se procurer (ou pour éviter) certaines caractéristiques de produits détermine largement l'évaluation du bien-être. Le défi central consiste à évaluer la valeur accordée par le consommateur à un bien dont le marché n'existe pas. Tel est généralement le cas en présence d'externalités et d'asymétries d'information, mais également lorsque les politiques commerciales en vigueur excluent les importations. La présentation complète de toutes les méthodes d'évaluation dépasserait la portée de la présente étude, et nous nous concentrons ici sur les évolutions les plus récentes, y compris en matière d'expériences économiques. On trouvera un excellent exposé des théories et méthodes dans OCDE (2006c). Comme cette publication n'évoque pas les méthodes expérimentales, nous avons quelque peu étoffé cette approche, de plus en plus utilisée et qui présente une pertinence toute particulière pour les questions abordées ici. 


\section{1. Évaluation des défaillances par le consommateur}

Il existe de nombreuses méthodes d'identification de la valeur accordée par les consommateurs pour des biens marchands ou non marchands (comme les externalités ou les biens environnementaux). Nombre d'entre elles sont potentiellement adaptées à l'analyse des mesures non tarifaires, car elles rendent compte du caractère hétérogène des préférences des consommateurs. La qualité de l'analyse coûts-avantages dépend considérablement de ces mesures. On peut employer deux types d'évaluation des biens non marchands, à savoir l'approche QALY, examinée à la section 6 ci-dessus, et l'approche PAP.

Les méthodes QALY peuvent être intégrées au cadre coûts-avantages proposé ici, en particulier en ce qui concerne les préjudices dont les consommateurs ne sont pas conscients (voir le graphique 3). Comme on l'a vu à la section 6 , cette approche ne permet pas de refléter les réactions des consommateurs dans la demande. En d'autres termes, les coûts estimés au moyen des méthodes QALY n'ont pas d'équivalent en termes d'ajustements de la demande liés à la réaction des consommateurs, comme ceux représentés dans les graphiques précédents.

À l'inverse, les méthodes reposant sur des estimations de la propension à payer (PAP) permettent aux économistes d'évaluer la réaction des consommateurs. Ces méthodes permettent d'inclure des aspects qualitatifs qui ne peuvent être traduits en termes de maladie identifiable à court terme.

La méthode de la dépense préventive cherche à mesurer la propension des agents à payer en observant les efforts fournis pour éviter la maladie. Avec cette méthode, une évaluation monétaire de la désutilité de l'état de maladie est ajoutée au coût estimé de cette dernière, ainsi qu'une estimation de la dépense préventive qu'un individu sera prêt à engager en fonction d'un degré de pathologie donné (Harrington et Portney, 1987).

Les méthodes d'évaluation contingente (contingent valuation) supposent d'interroger directement les individus à propos de leur propension à payer pour réduire le risque de maladie, ou, plus généralement, pour obtenir un bien de meilleure qualité. Les expériences de choix (choice experiments) déterminent indirectement la propension à payer au moyen d'une estimation économétrique reposant sur différents choix faits par les consommateurs. L'économie expérimentale (qui comprend les expériences de laboratoire, sur le terrain ou naturelles) place un groupe d'individus dans une situation où leur comportement réel est simulé (laboratoire) ou influencé (sur le terrain) afin de révéler leur propension à payer pour des qualités données (voir Lusk et Shogren, 2007a, pour une présentation exhaustive). Un des avantages de la méthode expérimentale réside dans la précision du contrôle de l'information révélée aux consommateurs, y compris avec une mesure de leur connaissance initiale au moyen de questionnaires ex ante et ex post. L'exemple numérique présenté plus bas utilise des mesures de la propension à payer obtenues par des expériences en laboratoire. Précisons que l'on peut estimer le supplément de prix applicable aux produits différenciés déjà vendus sur un marché donné au moyen d'une méthode économétrique reposant sur les prix hédoniques. Tel est par exemple le cas de l'évaluation de l'étiquetage écologique (voir Nimon et Beghin, 1999, et Teisl et al., 2002).

Avant de détailler brièvement certaines de ces méthodes d'évaluation de la propension à payer, il est particulièrement important de réfléchir à leur fiabilité, et en particulier à l'incitation de certaines personnes interrogées à sur- ou à sous-estimer leur propension à payer (voir Shogren, 2006). Différentes méthodes PAP ont été comparées de 
manière à faire apparaître les biais (voir une enquête in Levitt et List, 2007). À partir d'une comparaison avec une expérience sur le terrain, Blumenschein et al. (2008) ont montré que le biais hypothétique lié à l'évaluation contingente pouvait être supprimé au moyen d'une déclaration de certitude figurant à la fin du questionnaire. List (2007) note que les expériences sur le terrain peuvent, malgré leurs limites, constituer des relais utiles entre les expériences en laboratoire et les situations réelles. Tout en restant relativement neuf, le recours à des méthodes d'expérimentation économique peut être considéré comme une avancée majeure en vue d'une estimation plus fiable de la propension à payer des consommateurs. Plusieurs études comportent une comparaison directe des résultats d'expériences menées en laboratoire et d'autres expériences (y compris celles sur le terrain, dans des magasins). Parmi celles-ci, Shogren et al. (1999) ont montré que les évaluations en laboratoire et le comportement de marché coïncidaient pour des niveaux de prix élevées, sans doute du fait de la sélection de consommateurs réellement intéressés. Lusk et Fox (2003) ainsi que Marette et al. (2008c) ont démontré que les évaluations sur le terrain étaient proches de celles réalisées en laboratoire. Chang et al. (2008) ont montré que pour la viande de bœuf hachée et la farine de blé, les expériences en laboratoire, pour lesquelles les participants devaient dépenser de l'argent réel, dépassaient en précision les approches par évaluation contingente en matière de prédiction des prix de vente au détail. Malgré leurs limites, les résultats des études expérimentales peuvent être considérés comme une bonne approximation des réactions des consommateurs ou des citoyens, une fois certains biais éliminés. L'un des principaux avantages de cette méthode par rapport aux autres réside dans le fait qu'elle permet un contrôle plus précis des situations de choix, et permet donc en principe de transposer les résultats à d'autres contextes.

Les méthodes expérimentales ont été largement employées ces derniers temps dans de nombreux domaines liés à la sécurité alimentaire ou aux préférences alimentaires pour certaines caractéristiques de produits tels que les OGM, les produits biologiques, le bœuf aux hormones, la valeur de la biodiversité, etc. (voir le tableau 1.1 in Lusk et Shogren (2007b) avec ses 113 références récentes). Pour les domaines de la sécurité alimentaire, les expériences font ressortir une préférence significative pour des produits sûrs (Hayes et al., 1995 pour les États-Unis ; Rozan et al., 2004 pour la France).

Les expériences sur des biens controversés, tels que les produits alimentaires irradiés ou les hormones de croissance comme la somatotropine bovine, sont particulièrement adaptées à l'analyse des interdictions d'importation (comme dans le graphique 1). Elles permettent en effet d'exprimer les préférences en fonction des différents pays et fournissent des données expérimentales lorsqu'un produit étranger n'est pas disponible sur le marché national. Parmi les très nombreuses études ou expériences menées récemment sur les OGM (voir Costa-Font et al. (2008) pour une enquête complète), plusieurs concernent les différences de contexte réglementaire à cet égard en Europe et aux États-Unis. Noussair et al. (2002) montrent qu'une majorité de consommateurs français n'a pas de préférence pour les produits avec et sans OGM, ce qui contredit la pratique réglementaire d'interdiction en vigueur. Huffman et al. $(2003,2007)$ font ressortir la sensibilité de la propension à payer en fonction de la source d'information (organisations non gouvernementales, multinationales ou organes de santé publique). Lusk et al. (2003) et (2006b) procèdent à une comparaison directe de la propension à payer entre les États-Unis et certains pays d'Europe, pour aboutir à un degré plus élevé de réticence aux OGM de la part des consommateurs européens. Ces résultats ont servi à évaluer les effets sur le bien-être du maintien de l'identification des produits contenant des OGM (voir par exemple Sobolevsky A., G. Moschini et H. Lapan, 2005). 
L'interdiction de commercialisation du bœuf aux hormones en Europe a inspiré une expérience spécifique : Alfnes et Rickertsen (2003) montrent que la majeure partie des participants en Norvège préféraient le bœuf élevé dans leur pays à celui importé. Si le bœuf aux hormones est celui qui suscitait l'offre moyenne la moins élevée, $28 \%$ des participants étaient tout de même indifférents, voire préféraient le bœuf américain aux hormones à celui non traité en provenance des États-Unis. Précisons que ce type de résultat expérimental justifierait de simplifier la situation en séparant les consommateurs en seulement deux groupes : les consommateurs ayant une préférence, et les indifférents.

Lagerkvist, Carlsson et Viske (2006) - à travers une expérience de choix - et Napolitano et al. (2008) - au moyen d'une expérience en laboratoire - ont évalué la propension à payer pour le bien-être des animaux (utilisation des hormones incluse). Certaines expériences étudient les effets du programme d'étiquetage indiquant le pays d'origine des produits mené aux États-Unis (voir Lusk et al. (2006a)). Certaines études font ressortir la propension à payer pour la biodiversité (Stoneham et al. 2003), même si la principale technique employée dans ce domaine est l'évaluation contingente.

En révélant directement la propension à payer, la méthode expérimentale permet d'obtenir une estimation monétaire de tous les avantages qui découlent d'une mesure de politique commerciale donnée. Précisons que la quasi-totalité des études à partir d'expériences en laboratoire se penchent sur la propension à payer, mais sans l'intégrer dans une analyse du bien-être. En estimant la valeur de l'information, Rousu et al. (2004, 2007) se rapprochent de l'évaluation du bien-être, mais sans lien vers un modèle calibré.

\section{2. Évaluation des externalités de production}

Il existe de nombreuses études scientifiques estimant l'impact de différents parasites et éléments pathogènes en agriculture (le compendium CABI, plusieurs articles des revues Weed Science, The Agronomy Journal, Weed Technology, parmi beaucoup d'autres). Les études du compendium CABI partent des informations scientifiques disponibles dans le monde entier et comprennent de très nombreuses données sur la perte de rendement issue d'une multitude d'éléments pathogènes et de parasites sur différentes cultures, sur la forêt, sur le bétail et sur l'aquaculture. Les publications du compendium passent en revue les études scientifiques des 40 dernières années.

Les analyses les plus récentes et les plus visibles des coûts induits par les parasites sont celles de Pimentel et al. (2000) et (2005). À partir d'un large panorama d'études écologiques et agronomiques, ces deux articles font ressortir les coûts pour l'agriculture et pour l'environnement d'une longue liste d'espèces envahissantes. Les incertitudes concernant les estimations pour ces dernières ont beau rester considérables, ces articles fournissent néanmoins une estimation des pertes de rendement ou d'autres variations de la fonction de production ou des coûts entraînées par ces parasites. Les articles de Pimentel et al. constituent une source utile pour paramétrer à la fois les pertes de production et celles qui sont associées à des questions de ressources collectives mondiales, comme la diminution de la biodiversité, l'augmentation du nombre d'espèces en danger et autres.

Des progrès considérables ont été réalisés récemment en intégrant des modèles épidémiologiques à l'analyse économique. Pendell et al. (2007) ont procédé à une analyse de cette sorte pour la région du Midwest américain afin d'étudier une éventuelle épidémie de fièvre aphteuse. Plusieurs travaux s'intéressent aux politiques optimales de quarantaine (surveillance, tests, suivi) dans les économies ouvertes ou régionales, en intégrant des 
modèles sophistiqués et réalistes des dynamiques d'infection et de leur dimension spatiale (Adamson et Cook, 2007; Bicknell et al., 1999; Cook, 2007; Kompas et Che, 2003 et Kompas et al., 2004). Ces analyses reposent sur un contrôle stochastique dynamique, sur des méthodes numériques, et parfois sur des modèles spatiaux afin de quantifier la diffusion et l'établissement des invasions et de calculer les niveaux de quarantaine optimaux à partir de critères de coûts-bénéfices. Les résultats de ces travaux peuvent aboutir à des fourchettes de valeurs pour des modèles plus simples intégrant des externalités telles que des modifications moins complexes de la demande.

Il existe un grand nombre d'études économiques sur l'infestation d'adventices et sur la manière de la contrer (voir par exemple Eisworth et van Kooten, 2002 ; Taylor et Burt, 1984 ; Jones et Medd, 2000 ; et également quelques références dans Pimentel et al., 2005). Par exemple, Eiswerth et van Kooten (2002) analysent l'impact sur l'offre de l'infestation d'adventices sur la perte de rendement et sur le coût de production de foin pour le bétail dans le cas d'une plante allogène, le chardon doré, et en examinant cinq réactions possibles à l'invasion. Ce type d'analyse fournit suffisamment d'informations pour évaluer la perte de rendement si rien n'est fait, ainsi que le coût de limitation de l'invasion. Ce sont les variables nécessaires à la mise en œuvre du cadre coûts-avantages. Il existe également de nombreuses études interdisciplinaires sur les pertes de rendement induites par de multiples plantes adventices et autres parasites, telles que Swinton et al. (1994) parmi bien d'autres.

Les échanges commerciaux comme vecteur d'invasion des adventices ont également fait l'objet de recherches. Costello et al. (2007), mais aussi d'autres, se sont penchés sur l'introduction de parasites dans les ports américains dans l'histoire, ainsi que sur le coût qui en découle en termes de bien-être. Levine et d'Antonio (2003) analysent la relation statistique entre les échanges commerciaux et les adventices pour un large éventail de parasites.

En ce qui concerne les produits génétiquement modifiés, Wolfenbarger et Phifer (2000), ainsi que Qaim et Matuschke (2005), ont passé en revue les preuves scientifiques disponibles du coût et des avantages de cultiver des OGM plutôt que des plantes traditionnelles, y compris les effets sur le rendement, les économies sur les pesticides et le coût écologique plus large relatif à des externalités renouvelables similaires aux ressources collectives mondiales auxquelles les consommateurs attachent une valeur. Piggott et Marra (2007) analysent le coût et les avantages pour l'exploitation agricole associés à deux types de politiques de remplacement de la politique de refuge et de la vaporisation de pesticides, au niveau de la ferme et pour une grande société de graines, pour la culture du coton OGM. Marra et Piggott (2006) s'intéressent aux avantages non pécuniaires associés à différentes cultures d'OGM par rapport aux cultures traditionnelles.

\subsection{Valeur accordée aux ressources collectives mondiales}

Essayer d'attribuer une valeur à l'évitement d'une mauvaise gestion de ressources considérées comme appartenant à la communauté internationale représente peut-être la tentative la plus controversée de tous les cas abordés dans la présente étude. Tandis que les pays s'accordent en principe sur des sujets tels que l'importance de prendre des mesures correctives pour éviter le réchauffement climatique ou contrer la perte de biodiversité, ils s'opposent en pratique au moment de mettre au point des mécanismes en faveur d'un comportement plus écologique. Les diverses valeurs accordées aux ressources collectives mondiales et la distribution inégale des coûts des mesures correctives forment le socle de difficiles négociations multilatérales sur ces sujets. 
Dans le contexte des ressources collectives mondiales, les économistes divisent généralement la valeur économique totale entre différentes «valeurs d'usage » (la valeur dérivée de l'usage d'une ressource). Ces dernières peuvent représenter soit des « valeurs d'existence » (une valeur positive est accordée à la simple présence d'un écosystème), soit des «valeurs de possibilité » (bien que la ressource ne soit pas utilisée pour le moment, la possibilité de l'utiliser ultérieurement a une certaine valeur).

Il existe actuellement de nombreuses études apportant des estimations de chacun de ces éléments, comme le montrent les références de la bibliothèque numérique des ressources communes de l'université de l'Indiana (http://dlc.dlib.indiana.edu/). La tentative la plus ambitieuse, mais pas dénuée de controverse, d'attribuer une valeur aux services mondiaux de l'écosystème est relatée dans Costanza et al (1997). L'étude en question estime les valeurs d'usage directes et indirectes de 17 services d'écosystème, tels que la réglementation du secteur gazier, l'approvisionnement en eau et le cycle nutritif, et elle s'appuie sur des données déjà publiées, complétées par quelques calculs supplémentaires.

S'il est possible de faire de l'externalité de ressource collective mondiale une «marchandise » en instaurant pour elle un marché réel, alors on peut observer directement la valeur que lui accordent les consommateurs, sans nécessairement recourir aux méthodes de valorisation hors marchés évoquées plus haut. La prime que les consommateurs du pays importateur sont prêts à payer pour des produits issus du développement durable, de préférence aux autres, est directement indicatrice de leur valorisation de la ressource collective mondiale concernée.

Larson (2003) propose une étude d'évaluation sur les questions de ressources collectives, conceptuellement très proche du cadre proposé ici. Ce travail montre comment l'étiquetage du café cultivé sous toile peut contribuer à internaliser les externalités d'environnement positives dans la demande du consommateur. Les effets positifs sur les populations d'arbustes et d'oiseaux sont attribués à la culture du café sous toile, à une échelle de production réduite et à des conditions de rendement inférieur.

Il existe des tentatives à grande échelle, et soutenues par les pouvoirs publics, de programmes d'étiquetage de bois tropicaux et de poissons de mer comme conformes au développement durable. L'Organisation internationale des bois tropicaux (International Tropical Timber Organisation) a indiqué dans une étude récente (ITTO, 2008) que les acheteurs des pays importateurs n'étaient généralement pas prêts à payer un prix nettement supérieur pour des produits certifiés. Les suppléments de prix observés peuvent descendre jusqu'à $2 \%$ pour les importations britanniques de meranti de sciage certifié MTCC en provenance d'Asie, pour monter jusqu'à 10-30\% pour les bois tropicaux certifiés utilisés dans les chantiers navals au Danemark. Cependant, aucun supplément de prix n'a été constaté sur le marché japonais. Une telle variation des primes payées peut s'expliquer en partie par les écarts de la valeur accordée à la forêt tropicale par les consommateurs des différents pays importateurs. Les différents suppléments de prix d'un pays exportateur à l'autre peuvent également provenir des incertitudes quant à la fiabilité de l'étiquetage à l'origine de ces écarts, en particulier en ce qui concerne les importations africaines.

L'existence d'un supplément de prix suppose un risque de spéculation. Les producteurs ont eu intérêt à économiser sur le coût supplémentaire d'un usage plus écologique des ressources, entre autres dû aux frais de certification et de préservation de l'identité, tout en continuant d'étiqueter leur produit comme issu du développement durable. Si le contrôle de l'étiquetage est imparfait, et que les consommateurs apprennent 
cette faille, alors la valeur de l'information véhiculée par l'étiquetage et par le programme de certification s'en trouvera affectée, ce qui peut inciter les pays importateurs à recourir à la place à des mesures non tarifaires. Par exemple, la Norvège a décidé en 2007 d'interdire tous les bois tropicaux pour les constructions publiques, quel que soit l'étiquetage.

\section{Vers une mise en application du cadre}

Cette section explique comment le cadre proposé dans la section 6 peut devenir opérationnel pour une analyse de la politique commerciale en vigueur. Nous progresserons ici à travers les étapes habituelles de la mise en place d'un modèle, de la spécification des formes fonctionnelles aux estimations de paramètres importants du modèle spécifié, avec les différents types de données requis pour calibrer le modèle, et leurs sources.

\subsection{Paramétrage}

Même si un produit doté d'une caractéristique donnée n'est pas disponible sur le marché intérieur, et donc qu'on ne peut observer de demande en ce qui le concerne, il est possible de calibrer les fonctions d'offre et de demande. La propension à payer pour la caractéristique spécifique peut être obtenue par évaluation contingente ou par des résultats d'expériences, comme décrit plus haut. Dans une situation d'autarcie ou de politique prohibitive interdisant les importations de produits étrangers, les paramètres des fonctions linéaires de demande et d'offre peuvent être calibrés en fonction des prix et des quantités du marché intérieur.

Sans introduction du produit étranger, les équations d'offre et de demande sur le marché intérieur sont représentées par des approximations linéaires, avec l'élasticité correspondante au point d'approximation. À partir de l'équation (2), la demande globale sans information (avec $I=0$ ) est définie $\operatorname{par} Q(p)=(a-p) / b$. Avec la quantité

observée $\hat{Q}$ vendue pendant une période donnée, le prix moyen $\hat{p}$ observé sur la même période et l'élasticité directe du prix $\varepsilon(=(d Q / d P)(P / Q))$ issue d'estimations économétriques, le calibrage aboutit à des valeurs estimées pour la demande égales à $1 / \tilde{b}=-\varepsilon \hat{Q} / \hat{p}, \quad a=\tilde{b} \hat{Q}+\hat{p}$. La section 8.2 ci-après mentionne plusieurs bases de données publiques d'élasticité que l'on peut exploiter.

Les estimations de la propension à payer issues d'expériences ou d'évaluations contingentes doivent être intégrées à la demande calibrée. Il est naturellement nécessaire au préalable que l'échantillon utilisé pour les expériences soit représentatif de la population globale analysée dans l'enquête ${ }^{13}$. À partir des résultats des expériences, on peut identifier la proportion de participants indifférents ou ayant une préférence.

Les résultats des expériences de choix peuvent être intégrés par la suite à une fonction de demande calibrée pour la proportion de consommateurs intéressés par, ou réticents à la consommation d'une caractéristique spécifique, d'une manière cohérente avec le cadre

13. Non sont pas prises en compte ici les expériences menées avec des étudiants dans les universités. 
défini par les équations (1) et (2) du point $6.1^{14}$. La révélation de l'information en laboratoire permet aux participants de connaître la caractéristique véhiculée par le produit étranger dans des conditions de libre-échange (avec ou sans étiquetage obligatoire). Cette manière de procéder permet d'isoler économétriquement la propension à payer pour la caractéristique spécifique. Naturellement, la variation de la propension à payer issue de l'expérience ne peut être utilisée de manière fiable dans le modèle calibré que si l'impact de l'information est statistiquement significatif.

Dans une situation de libre-échange, la caractéristique indésirable du produit étranger est indiscernable pour les consommateurs ayant une préférence, ce qui entraîne une contraction de la courbe de la demande au point d'équilibre initial, en ligne avec les modèles de Polinsky et Rogerson (1983) et de Lichtenberg et al. (1998). Les variations relatives de la propension à payer observées dans l'expérience et dépendant de la caractéristique supplémentaire servent à déterminer la variation de la demande (voir Marette et al., 2008a pour davantage de détails).

La variation de la demande reposant sur l'expérience en laboratoire est mesurée comme un infléchissement vertical de la demande, ce qui donne la droite $D_{2}$ dans le graphique 2, avec $\delta=\left[E\left(W T P^{h h}\right)-E\left(W T P^{h}\right)\right] / E\left(W T P^{h}\right)$, où $E($.$) représente la valeur$ moyenne de la propension à payer pour les sujets étudiés qui modifient leur propension à payer de manière significative une fois révélée l'information sur la caractéristique du produit importé (à savoir que $W T P^{h h} \neq W T P^{h}$ ), $h$ correspond à la situation antérieure à la révélation de l'information, et $h h$ dénote la situation suite à cette révélation. La variation relative $\delta$ isole la propension à payer relative pour la caractéristique additionnelle, indépendamment de la dotation initiale ou de la valeur originelle du produit proposé pendant l'expérience. Sachant que le modèle est calibré pour un prix initial $p^{A}$ et intégré à l'analyse coûts-avantages, la valeur de la variation de la demande correspond à $r_{2}=-\delta p^{A}$. Ce résultat est à incorporer dans l'équation (2), avec un infléchissement de la demande pour $\delta<0$ et un redressement de cette dernière pour $\delta>0$. Cette valeur mesure la variation de la demande calibrée au prix $p^{A}$. Notons qu'il est également possible d'estimer avec cette mesure la composante de bien-être calculée auprès de consommateurs indifférents (voir Marette et al. (2008b) pour davantage de détails).

\section{2. $\quad$ Sources des données}

\section{Mesures de la propension à payer}

Comme nous l'avons expliqué dans la section 7 , les mesures de la propension à payer obtenues par évaluation contingente ou par des expériences en laboratoire peuvent être estimées directement, ou bien être déduites d'une méta-analyse des résultats de travaux publiés. Les paramètres restants peuvent souvent être obtenus à partir de sources externes. Lorsque l'on utilise des données secondaires, il est important de tenir compte du contexte spécifique et du pays dans lesquels ont été réalisées ces estimations. Les estimations de la propension à payer obtenues dans un pays donné et dans un contexte particulier pourront n'être pas directement transposables à d'autres situations, et il pourra s'avérer nécessaire de procéder à des ajustements pour tenir compte de ces différences.

14. Précisons que ce groupe pourrait également être divisé en plusieurs sous-groupes en fonction de l'importance des variations de la propension à payer. 


\section{Élasticités}

Les élasticités de prix et de revenu de l'offre et de la demande sont disponibles dans deux bases de données largement employées pour la modélisation de l'équilibre partiel : FAPRI (http://www.fapri.iastate.edu/tools/elasticity.aspx) et USDA-ERS (http://www.ers.usda.gov/Data/InternationalFoodDemand/. La base de données qui sous-tend le modèle conjoint Aglink-Cosimo de l'OCDE et de la FAO contient un très grand nombre d'estimations de l'élasticité (OCDE (2007b) et http://www.agrioutlook.org/pages/0,2987,en_36774715_36775671_1_1_1_1_1,00.html. On trouve des estimations récentes des élasticités de la demande à l'importation dans Kee et al. (2008) qui évaluent ces élasticités pour des produits désagrégés au niveau de leur code SH 6 pour un large ensemble de pays.

\section{Données sur les politiques et les dissensions commerciales}

À ce jour, deux bases de données jouent un rôle central dans l'analyse de la réglementation des obstacles techniques au commerce (OTC) et des mesures sanitaires et phytosanitaires. Premièrement, les notifications de l'OMC sur les mesures non tarifaires sont collectées et traitées par la Conférence des Nations unies sur le commerce et le développement (CNUCED) dans sa base de données TRAINS. La CNUCED complète les notifications à partir de sources nationales et classe les différentes mesures selon son propre système, qui comprend : les mesures para-tarifaires, les mesures de contrôle des prix, les mesures financières, le régime de licence automatique, les mesures de limitation quantitative, les mesures monopolistiques et les mesures techniques. Ces catégories sont ensuite encore développées selon une ramification plus fine (voir http://r0.unctad.org/trains_new/tcm_link.shtm). La base de données TRAINS est consultable (service payant) via le système WITS de la Banque mondiale (http://wits.worldbank.org/witsweb/). WITS publie les données au niveau des matières premières HS-6 et permet d'identifier plusieurs fondements officiels des politiques suivies (santé publique, protection de l'environnement, etc.). Cette base de données a été récemment utilisée, entre autres, par Disdier et al. (2008), par Fontagné et al. (2005) et par Henry de Frahan et Vancauteren (2006). Ces ensembles de données présentent comme inconvénient de présenter des informations inégalement réparties entre les pays et une couverture hétérogène des mesures prises pour les différents pays et matières premières. Ils peuvent renseigner davantage sur les comportements que sur la réglementation. Pour une discussion à propos de cette base de données, voir OCDE (2008c).

La seconde base de données importantes utilisée récemment s'appelle Perinorm (www.perinorm.com) ; c'est une base de données bibliographiques fournie par les agences de normalisation industrielle britannique, allemande et française (BSI, DIN et AFNOR). Elle fournit des informations sur les normes publiques, dont le respect s'entend sur une base volontaire, et sur les réglementations techniques, qui sont obligatoires. De ce fait, elle couvre aussi bien les normes obligatoires que les normes facultatives, nationales, européennes et internationales, en provenance de 23 pays, pour aboutir à un total de plus de 1100000 entrées sur des normes australiennes, des pays de l'UE, japonaises et américaines. Cette base de données a été consultée par Moenius (1999) et (2006) ainsi que par Czubala, et al. (2007), qui ont ingénieusement utilisé les données fournies pour distinguer les normes bilatérales partagées de celles qui ne le sont pas (Moenius) ou les normes internationales des normes non harmonisées (Czubala et al., 2007). Czubala et al. s'appuient sur Perinorm en association avec le catalogue en ligne du Comité européen de 
normalisation pour créer une base de données originale de normes de produits dans l'UE dans les domaines du textile et de l'habillement. Une distinction est faite entre les normes « harmonisées », équivalentes à des normes ISO, et celles qui ne le sont pas. L'intégration de normes volontaires dans Perinorm représente un avantage par rapport aux informations sur les notifications présentées par la CNUCED, qui ne comprennent que les OTC à caractère obligatoire, mais la couverture géographique est beaucoup plus limitée.

Le nouveau système de gestion des renseignements SPS créé par l'OMC (http://spsims.wto.org/) offre un accès aux mesures sanitaires et phytosanitaires notifiées à l'OMC, ainsi qu'aux préoccupations commerciales officielles communiquées par les gouvernements Membres au comité SPS de cette dernière. SPS IMS fournit essentiellement un accès en ligne à des informations textuelles. La version publique du système apporte des informations, des questions et des notifications sur les normes sanitaires et phytosanitaires, en fonction de leurs besoins spécifiques, sur la base d'une recherche multicritères (regroupements géographiques, codes produits, périodes de commentaires et mots-clés). La section SPS du site de l'OMC représente une source majeure d'information sur les normes obligatoires et les litiges dans ce domaine. Ces données sur les notifications de SPS sont également accessibles via la base de données Inquit (www.inquit.com), qui permet de consulter aisément les mesures sanitaires et phytosanitaires de septembre 1999 à mai 2007. Les données sur les litiges à l'OMC ont été compilées et analysées par (et sont consultables dans) Horn et Mavroidis (2006).

Le Portail international de la sécurité sanitaire des aliments et de la santé animale et végétale (IPFSAPH, http://www.ipfsaph.org/Fr/default.jsp) publie en accès libre des informations sur les dispositions légales relatives à la sécurité alimentaire. L'IPFSAPH est le fruit d'une coopération entre plusieurs organismes de normalisation et agences internationales reconnus par l'Accord SPS. Le Portail a été mis au point par la FAO, en association avec Codex Alimentarius, le secrétariat de la CIPV et l'OIE. La base de données contient des dispositions légales internationales obligatoires relatives à la sécurité alimentaire, des instruments internationaux (tels que ceux mis au point par les organismes de normalisation comme l'OIE ou Codex Alimentarius), des instruments régionaux (essentiellement des règlements de l'Union européenne) et des dispositions non contraignantes (telles que les considérations de l'OCDE sur la sécurité dans les biotechnologies de 1992 ou le Code de conduite pour une pêche responsable de la FAO). Le portail propose un moteur de recherche par produit, par pays et par sujet multisectoriel (tel que l'impact sur la santé humaine).

La Banque mondiale dispose également d'une base de données mise au point par Wilson and Associates sur les mesures visant à faciliter les échanges, qui s'appuie sur les résultats d'enquêtes du Forum économique mondial. Enfin, l'enquête de la Banque mondiale intitulée World Bank Technical Barriers to Trade Survey (Wilson et Otsuki, 2004b) (http://www1.worldbank.org/wbiep/st-db/) constitue la première tentative d'enquête à l'échelle mondiale sur l'impact des normes techniques. Elle rassemble des informations collectées auprès d'entreprises agricoles, manufacturières et commerciales de différents pays émergents et concerne les obstacles techniques rencontrés sur les marchés d'exportation ainsi que leur impact sur la réussite de ces entreprises à vendre à l'étranger. Les données rassemblées couvrent 689 entreprises de plus de 20 secteurs, dans 17 pays en développement. 


\section{Exemple : l'étiquetage du poisson}

L'exemple ci-après illustre l'application du cadre coûts-avantages pour l'évaluation des mesures non tarifaires, en évaluant l'impact de l'étiquetage obligatoire du poisson destiné à la consommation en France. Il est tiré de Marette et al. (2008a). Il s'agit ici d'évaluer l'impact d'un étiquetage mentionnant des informations sanitaires censées guider le choix du consommateur entre un type de poisson relativement "risqué » (le thon) et un autre, non seulement «moins risqué », mais qui présente en outre des bénéfices pour la santé (la sardine). Sous l'angle de la politique sanitaire, une réorientation de la consommation vers la sardine est souhaitable car, relativement au thon, cette dernière contient davantage d'acides gras oméga 3 , qui sont bons pour la santé, et moins de méthylmercure malsain. Cependant, les politiques incitant à remplacer le thon ont des implications en termes de commerce international, puisque l'essentiel du thon en boîte $(95 \%)$ et des sardines en conserve (99\%) consommés en France sont importés. En particulier, les producteurs étrangers de thon verraient leur demande diminuer suite à la révélation du risque sur leur produit (comme dans le graphique 3), ce qui pourrait avoir un impact significatif sur les pays en développement exportateurs de ce poisson, tels que la Côte d'Ivoire ou les Seychelles (voir Ofimer, 2007). Cet exemple correspond à une évaluation du bien-être très similaire à la comparaison des graphiques 2 et 3 ci-dessus. La possibilité d'une norme prohibitive, qui reviendrait à interdire l'importation de thon, n'est pas envisagée ici, mais se rapprocherait, sur le plan conceptuel, de la comparaison des graphiques 1 et 2 ci-dessus. L'impact d'une interdiction prohibitive sur le prix serait probablement important.

Une expérience de choix en laboratoire a été organisée pour connaître la valeur accordée par les consommateurs aux qualités sanitaires d'un produit et pour évaluer la valeur de l'information sur ce dernier en lien avec un étiquetage obligatoire. Les sujets invités à participer étaient des Françaises en âge d'avoir des enfants, du fait de l'importance particulière du poisson pendant la grossesse. Conformément à la méthode décrite à la sous-section 8.1 , les résultats de l'expérience ont été calibrés en fonction de l'élasticité et des données du marché pour déterminer la valeur de l'information. Par rapport au cadre décrit dans la présente étude, l'expérience présentait comme facteur supplémentaire la présence de deux biens (le thon et la sardine), substituts de consommation.

Les paramètres du modèle sont initialement calibrés de manière à reproduire les prix et quantités constatés en France pendant l'année 2002 (voir le tableau 2), l'année complète la plus récente à la date de l'analyse. Pour l'offre, une fonction d'offre linéaire est calibrée en fonction des estimations d'élasticité calculées par les chercheurs. Le calibrage de la fonction de demande linéaire se fonde sur les estimations d'élasticité des prix simple et croisée pour le thon comme pour la sardine. Trois schémas de demande sont mis en place pour la situation initiale, sans information sur les caractéristiques des produits : 1) un groupe de consommatrices qui achète aussi bien du thon que des sardines ; 2) un groupe de consommatrices qui n'achète que du thon et 3 ) un groupe de consommatrices qui n'achète que des sardines. Le cumul des demandes des différents sous-groupes correspond à la demande totale. 
Tableau 2. Spécification de la demande de thon en boîte et de sardines en conserve en France

\begin{tabular}{|c|c|c|c|}
\hline Variables & Description & Valeurs & \\
\hline \multirow[t]{3}{*}{ Thon en boîte } & Consommation totale en France en 2002 (en tonnes) & 63845 & \\
\hline & Prix moyen en 2002 (en euros) & 6.1 & \\
\hline & Élasticité de l'offre & 0.2 & \\
\hline \multirow[t]{3}{*}{$\begin{array}{l}\text { Sardines en } \\
\text { conserve }\end{array}$} & Consommation totale en France en 2002 (en tonnes) & 11484 & \\
\hline & Prix moyen en 2002 (en euros) & 8.2 & \\
\hline & Élasticité de l'offre & 0.2 & \\
\hline \multicolumn{4}{|c|}{$\begin{array}{ll}\text { Consommateurs 1) qui achètent aussi bien du thon que des sardines }\end{array}$} \\
\hline \multicolumn{2}{|c|}{$\%$ des ménages consommant des sardines et du thon ${ }^{3}$} & $65 \%$ & \\
\hline \multicolumn{2}{|c|}{ Élasticités de la demande } & Thon & Sardines \\
\hline \multicolumn{2}{|c|}{ Élasticité-prix simple } & $-0.58^{*}$ & $-0.476^{*}$ \\
\hline \multicolumn{2}{|c|}{ Élasticité-prix croisée } & $-0.059^{*}$ & $0.144^{*}$ \\
\hline \multicolumn{4}{|c|}{ Consommateurs 2 2) qui n'achètent que du thon } \\
\hline \multicolumn{2}{|c|}{$\%$ des ménages ne consommant que du thon } & $32.5 \%$ & \\
\hline \multicolumn{2}{|r|}{ Élasticité-prix simple de la demande } & $-0.534^{*}$ & \\
\hline \multicolumn{4}{|c|}{$\begin{array}{ll}\text { Consommateurs } & \text { 3) qui n'achètent que des sardines }\end{array}$} \\
\hline \multicolumn{2}{|c|}{$\%$ des ménages ne consommant que des sardines } & $2.5 \%$ & \\
\hline \multicolumn{2}{|r|}{ Élasticité-prix simple de la demande } & $-0.451^{*}$ & \\
\hline
\end{tabular}

Source : Marette et al. (2008a). Pour l'estimation de l'élasticité, * dénote un niveau de signification de $5 \%$ de la régression entre l'échelle logarithmique des quantité et celle des prix.

La demande initiale permet de calculer le bien-être en l'absence de message sanitaire. L’impact sur le bien-être de ce message est calculé à partir des résultats de l'expérience en laboratoire pour mettre la demande à jour une fois l'information sur la santé révélée.

On suppose que seule la demande des consommateurs achetant les deux types de poissons sera modifiée par le message sanitaire ${ }^{15}$. On peut observer la réaction à l'information sur le produit à travers le comportement de choix. D'après l'expérience et la définition de $\delta$ au point 8.1 , la croissance moyenne de la propension à payer moyenne pour la sardine serait égale à $\bar{\delta}_{\text {sardine }}=1.28$ après perception de l'information, ce qui se traduit par une augmentation de la demande (voir Marette et al., 2008a). La variation moyenne de la propension à payer relative pour le thon correspond à $\bar{\delta}_{\text {tuna }}=-0.21$ après perception de l'information, ce qui entraîne un infléchissement de la demande. Ces valeurs issues de l'échantillon expérimental sont extrapolées à la population française correspondante pour calibrer les variations de la demande de thon et de sardines. Pour isoler le sous-groupe de consommateurs «exposés » ou "ayant une préférence », des « indifférents » (qui ne se sentent pas concernés par le message sanitaire de l'expérience),

15. On pourrait aussi prévoir la possibilité que les consommateurs qui achètent uniquement du thon et ceux qui achètent uniquement des sardines modifient leur consommation, mais la procédure expérimentale basée sur la substitution entre ces produits n’a pas été élaborée pour ce cas. 
l'analyse suppose que seuls les ménages comportant des femmes en âge d'avoir des enfants, ou ayant des enfants de moins de 14 ans, seront pertinents (seules les femmes en âge d'avoir des enfants ont participé à l'expérience, car c'est ce groupe que ciblent les autorités responsables de la sécurité alimentaire). Cette distinction est opérée sur la foi de preuves médicales qui montrent clairement que ce groupe est exposé à un risque plus élevé. Le groupe de ménages «exposés » représente $50.5 \%$ des consommateurs français. Le reste de la population est considéré comme étant complètement indifférent, avec $\delta_{\text {sardine }}=0$ et $\delta_{\text {tuna }}=0$, ce qui correspond à une hypothèse restrictive.

Afin de mesurer les différentes formes de diffusion de l'information, on a opéré une distinction selon que la totalité ou la moitié des $50.5 \%$ de ménages concernés recevaient un message sanitaire. La variation des profits des producteurs étrangers de thon et de sardines en boîte peut être isolée des bénéfices de la chaîne d'approvisionnement (industrie de la conserve et distributeurs sur le marché national). L'estimation des bénéfices des producteurs étrangers tient compte de la part des importations dans la consommation nationale de conserves de poisson (à savoir $95 \%$ pour le thon et $99 \%$ pour la sardine) mais également de la part de l'industrie de la conserve dans la valeur ajoutée totale de la chaîne d'approvisionnement. À partir des prix à l'importation et des prix de vente finaux, la part estimée du prix à la consommation perçu par l'industrie du thon en boîte ressort à $48 \%$, contre $37 \%$ pour la sardine en conserve. Le reste de la valeur ajoutée totale revient aux distributeurs français. Intéressons-nous à présent aux résultats.

Le tableau 3 présente l'impact économique de l'information sur les prix et sur le surplus des agents pour l'année 2002. La variation de la demande suppose une baisse du prix d'équilibre du thon $\left(\Delta p_{T}<0\right)$ mais une augmentation de celui de la sardine $\left(\Delta p_{S}>0\right)$. La variation de prix des sardines est plus importante que la variation de prix en valeur absolue pour le thon, du fait d'un accroissement de la demande plus important pour les premières par rapport à la diminution de la demande du second. De ce fait, les profits des producteurs de thon reculent, tandis que ceux des producteurs de sardines progressent. Les producteurs de thon étrangers pâtissent de la diffusion de l'information, mais cette perte est compensée par le bénéfice apporté aux producteurs de sardines étrangers, puisque la variation des bénéfices étrangers totaux est positive. Les distributeurs qui perçoivent plus de la moitié de la valeur ajoutée dans la chaîne d'approvisionnement $(52 \%$ du prix à la consommation pour le thon et $63 \%$ pour les sardines) tirent profit de la diffusion de l'information. Les consommateurs des ménages exposés bénéficient de la diffusion de l'information, comme le montre la variation positive de leur surplus de consommateurs. Ce groupe profite de la possibilité de prendre des décisions de consommation en meilleure connaissance de cause. La variation de surplus de ces consommateurs concernés est un peu plus élevée lorsque la moitié des consommateurs concernés reçoivent des informations par rapport au scénario où les informations sont diffusées à tous les consommateurs concernés ( 8.4 millions EUR contre 7.6 millions EUR). De fait, l'avantage procuré par des informations supplémentaires provient essentiellement des informations sur le contenu en oméga 3 des sardines. Toutefois, l'effet marqué de hausse des prix des sardines annule partiellement les bénéfices induits par la diffusion de l'information. Les consommateurs non concernés par l'information pâtissent de la variation subséquente des prix du marché, avec une variation négative de leur surplus de consommateurs. Cette perte provient de la forte augmentation des prix des sardines, qui occulte l'effet positif de la légère diminution des prix du thon. Étant donné que les consommateurs non concernés consomment principalement du thon, 
la variation de surplus de ces consommateurs est d'une amplitude similaire dans les deux scénarios, car la variation de prix du thon est relativement faible compte tenu d'une demande relativement non élastique. Le tableau 3 fait apparaitre un gain net de bien-être issu de l'information des ménages exposés, malgré quelques pertes pour les producteurs de thon et pour les consommateurs qui ne se sentent pas concernés par le message sanitaire.

Tableau 3. Effets économiques de l'étiquetage (message d'information) sur les prix et sur les différents groupes de consommateurs (en euros)

\begin{tabular}{|c|c|c|c|c|}
\hline & & Unité & $\begin{array}{c}\text { Tous les } \\
\text { consommateurs } \\
\text { concernés reçoivent } \\
\text { l'information } \\
\text { (50.5\% de la population) } \\
\end{array}$ & $\begin{array}{l}\text { La moitié des } \\
\text { ménages concernés } \\
\text { reçoivent l'information } \\
(25.25 \% \text { de la } \\
\text { population) } \\
\end{array}$ \\
\hline \multicolumn{5}{|c|}{ Variations de prix } \\
\hline$\Delta p_{T}$ & Thon, en valeur absolue & euros & -0.28 & -0.14 \\
\hline$\Delta p_{T} / \hat{p}_{t}$ & Thon, en valeur relative & $\%$ & $-4.6 \%$ & $-2.3 \%$ \\
\hline$\Delta p_{s}$ & Sardine, en valeur absolue & euros & 3.70 & 1.85 \\
\hline$\Delta p_{s} / \hat{p}_{s}$ & Sardine, en valeur relative & $\%$ & $45.2 \%$ & $22.6 \%$ \\
\hline \multicolumn{5}{|c|}{ Variation de surplus en cas de variation des prix } \\
\hline$\Delta \Pi_{T}$ & $\begin{array}{l}\text { Variation des bénéfices pour } \\
\text { l'ensemble de la chaîne } \\
\text { d'approvisionnement du thon } \\
\text { en boîte }\end{array}$ & euros & -21579855 & -10832098 \\
\hline$\Delta \Pi_{T}^{F}$ & $\begin{array}{l}\text { Variation des bénéfices des } \\
\text { producteurs étrangers }\end{array}$ & euros & -9977086 & -5008039 \\
\hline$\Delta \Pi_{S}$ & $\begin{array}{l}\text { Variation des bénéfices pour } \\
\text { l'ensemble de la chaîne } \\
\text { d'approvisionnement de la } \\
\text { sardine en boîte }\end{array}$ & euros & 54931214 & 26503449 \\
\hline$\Delta \Pi_{S}^{F}$ & $\begin{array}{l}\text { Variation des bénéfices des } \\
\text { producteurs étrangers }\end{array}$ & euros & 20444841 & 9864315 \\
\hline$\Delta \Pi_{S}^{F}+\Delta \Pi_{T}^{F}$ & $\begin{array}{l}\text { Variation des bénéfices } \\
\text { totaux des producteurs } \\
\text { étrangers }\end{array}$ & & 10467755 & 4856276 \\
\hline$\Delta C S_{1}$ & $\begin{array}{l}\text { Variation de surplus des } \\
\text { consommateurs indifférents }\end{array}$ & euros & -13830515 & -12576828 \\
\hline$\Delta C S_{2}$ & $\begin{array}{l}\text { Variation de surplus des } \\
\text { consommateurs concernés }\end{array}$ & euros & 7609339 & 8403685 \\
\hline$\Delta W^{O}$ & Bien-être national & & 16662428 & 6641931 \\
\hline$\Delta W$ & $\begin{array}{l}\text { Bien-être total (bien-être } \\
\text { national + bénéfices } \\
\text { étrangers) }\end{array}$ & euros & 27130183 & 11498207 \\
\hline$\Delta W / W(\%)$ & $\begin{array}{l}\text { Variation relative du bien- } \\
\text { être total }\end{array}$ & $\%$ & $2.8 \%$ & $1.2 \%$ \\
\hline
\end{tabular}

Sources: Marette et al. (2008a) et estimations par les auteurs des bénéfices des producteurs étrangers. 
Notons que cette analyse pourrait être étendue en y incluant d'autres instruments non tarifaires, tels que la mise en place d'une norme obligatoire concernant le mercure dans le thon, ou encore des mesures fiscales ou des subventions (Marette et al., 2008b). Comme nous l'avons souligné plus haut, les résultats de ce type d'analyse dépendent de la configuration des paramètres et de la qualité des données utilisées. Pour déterminer la fiabilité des résultats et mettre en avant les limites de l'étude, on peut procéder à une analyse de sensibilité au moyen d'autres hypothèses, de fourchettes de valeur des paramètres différentes et de scénarios distincts. Il peut s'avérer plus intéressant de démontrer la solidité d'une conclusion obtenue au moyen d'une fourchette de paramètres plausibles que d'une seule estimation positive du bien-être. Ce simple exemple illustre le caractère applicable, la faisabilité et la flexibilité du cadre proposé.

\section{En conclusion}

La présente étude ouvre la voie vers une analyse systématique des coûts et des avantages économiques des mesures non tarifaires. La méthodologie proposée fonctionne pour la comparaison de différents choix politiques tels que la mise en place de normes, de contrôles aux frontières et d'étiquetage, dans un contexte international. Elle contribue à analyser les mesures non tarifaires de manière plus complète qu'en s'intéressant aux seuls effets commerciaux.

Les coûts d'efficience des mesures non tarifaires sont bien moins évidents que les pertes de bien-être associées aux obstacles non commerciaux liés aux tarifs douaniers et aux contingents. Les mesures non tarifaires ne comportent pas nécessairement les inefficiences économiques associées aux obstacles commerciaux traditionnels, à moins qu'elles n'opèrent une discrimination entre les différentes sources d'approvisionnement. À priori, on ne peut dire clairement si la réglementation a un impact sur les échanges, ou si la suppression de mesures non tarifaires associées préjudiciables aux échanges permettrait de réaliser des gains d'efficience supérieurs aux pertes induites par une réglementation moins puissante.

L'approche comparative proposée des mesures non tarifaires permet d'identifier différentes manières de réagir à un problème de réglementation donné. En énumérant systématiquement les coûts et les avantages pour les différents agents économiques intéressés, il est possible de suivre une approche reposant sur des preuves et formant une base solide d'échanges mutuels et d'identification des solutions les moins coûteuses. Cette approche est d'autant plus importante compte tenu de la multiplication des différends commerciaux liés à l'innocuité et à la qualité des produits alimentaires (Josling et al. 2004).

La méthodologie proposée ouvre également des pistes d'analyse à partir de la classification des mesures non tarifaires récemment proposée par l'équipe MAST.

L'application du cadre proposé à différents cas présentant différentes politiques commerciales et sur différents marchés donne une idée de son caractère applicable et de ses limites dans différentes circonstances, et indique si le modèle est en mesure de fournir des estimations fiables de l'ensemble des avantages et des coûts. Deux documents (TAD/TC/CA/WP(2008)4 et TAD/TC/CA/WP/RD(2008)1) préparés pour le Groupe de travail mixte sur l'agriculture et les échanges sont consacrés à la disponibilité des données et à la sélection des cas qui pourraient donner lieu à une analyse plus approfondie. 


\section{Bibliographie}

Adamson, D., et D. Cook. (2007). «Re-examining economic options for import risk assessments », document Mimeo présenté au congrès annuel 2007 de l'AARES à Queenstown, Nouvelle-Zélande.

Akerlof, G. (1970). The market for 'lemons'; Qualitative uncertainty and the market mechanism. Quarterly Journal of Economics, 84, 488-500.

Alfnes, F., et K. Rickertsen (2003). European Consumers' Willingness to Pay for U.S. Beef in Experimental Auction Markets. American Journal of Agricultural Economics, 85:2 396.

Antle, J. (2000), « $\mathrm{n}^{\circ}$ such thing as free safe lunch: the costs of food safety regulation in the meat industry », American Journal of Agricultural Economics, Vol.82 n², p.310-322.

Barrett, C.B., et Y.N. Yang. 2001. Rational incompatibility with international product standards, Journal of International Economics 54(1): 171-191.

Baumol W.J., et W.E. Oates. (1988). The Theory of Environmental Policy Cambridge University Press.

Beghin J. (2006), « Nontariff Barriers », dans S. Darlauf et L. Blume (dir. pub.), The New Palgrave Dictionary of Economics, 2nd Edition, Palgrave Macmillan LTD, 2008: 126-129.

Bicknell, K., Wilen, J.E. et Howitt R.E. (1999). Public policy and private incentives for livestock disease control, Australian Journal of Agricultural and Resource Economics, 43, 501-521.

Blumenschein, K., G. Blomquist, M. Johannesson, N. Horn, et P. Freeman (2008). Eliciting Willingness to Pay Without Bias: Evidence from a Field Experiment. Economic Journal, 118 , 114-137.

Boughner, D.S., H. de Gorter, et I. M. Sheldon. (2000). The Economics of Two-Tier Tariff-Rate Import Quotas in Agriculture, Agricultural and Resource Economics Review 29/1: 58-69.

Bureau, J. C., S. Marette, et A. Schiavina. Non-Tariff Trade Barriers and Consumers' Information: The Case of EU-US Trade Dispute on Beef . European Review of Agricultural Economics, 254 (1998): 437-462.

Buzby, J.C., T. Roberts, C.J. Lin, et J. MacDonald. (1996), Bacterial Foodborne Disease: Medical Costs and Productivity Losses, U.S. Department of Agriculture, Economic Research Service, Agricultural Economic Report 741.

CABI. CABI Aquaculture_Compendium disponible en ligne à l'adresse : http://www.cabi.org/compendia/ac/index.asp.

CABI Animal Health and Production Compendium, disponible en ligne à l'adresse : http://www.cabi.org/compendia/ahpc/.

------. CABI Crop Protection Compendium, disponible en ligne à l'adresse : http://www.cabi.org/compendia/cpc/.

------ CABI Forestry Compendium, disponible en ligne à l'adresse : http://www.cabi.org/compendia/fc/.

Casella, A. (1996). «Free Trade and Evolving Standards », chapitre 3 dans J.N. Bhagwati et R.E. Hudec (dir. pub.), Fair Trade and Harmonization: Prerequisites for Free Trade? MIT Press.

Centre for International Economics (2006). Feasibility of extending CoOL: A benefit cost analysis, Canberra \& Sydney: CIE.

Chang J., J. Lusk et B. Norwood (2008). « How Closely Do Hypothetical Surveys and Laboratory Experiments Predict Field Behavior? », document de travail, Oklahoma State University. 
Cheney, T. (2005), Distorted gravity: heterogeneous firms, market structure and the geography of international trade, mimeo, Massachusetts Institute of Technology (MIT), Cambridge/Massachusetts.

Chichilnisky, G. (1994). North-South Trade and the Global Environment, The American Economic Review, Vol. 84, n 4 (septembre 1994), pp. 851-874.

Conseil du Trésor du Canada (2007). Guide d'analyse coûts-avantages pour le Canada : Propositions de réglementation. http://www.regulation.gc.ca/documents/gl-ld/analys/analys01eng.asp

Costa-Font, M., J. M. Gil et W. B. Traill (2008). «Consumer acceptance, valuation of and attitudes towards genetically modified food: Review and implications for food policy." Food Policy, 33, 99-111.

Costanza, Robert, Ralph d'Arge, Rudolf de Groot, Stephen Farber, Monica Grasso, Bruce Hannon, Karin Limburg, Shahid Naeem, Robert V. O'Neill, Jose Paruelo, Robert G. Raskin, Paul Sutton, Marjan van den Belt, 1997, The value of the world's ecosystem services and natural capital, Nature, vol. 387, 15 mai 1997, pp. 253-260.

Costello, C., M. Springborn, C. McAusland, et A. Solow. 2007. «Unintended biological invasions: Does risk vary by trading partner? » Journal of Environmental Economics and Management 54(3): 262-276.

Crespi J. et S. Marette. How Should Food Safety Certification Be Financed. American Journal of Agricultural Economics, 83, 4 (2001): 852-861.

Czubala, W., B Shepherd, et J.S. Wilson. 2007. « Help or Hindrance? The Impact of Harmonized Standards on African Exports », Policy Research Working Paper, Banque mondiale 4400, Washington DC.

Disdier, A.C., L. Fontagné, et M. Mimouni. 2008. The Impact of Regulations on Agricultural Trade: Evidence from SPS and TBT Agreements, American Journal of Agricultural Economics 90(2): 336-350

Eiswerth, M.E., et G.C. van Kooten. (2002), Uncertainty, Economics, and the Spread of an Invasive Plant Species, Amer. Journal of Agricultural Economics, 84 (5): 1317-1322.

Ferrantino, M. (2006), Évaluation quantitative des effets économiques et commerciaux des mesures non tarifaires, Document de travail $n^{\circ} 28$ de l'OCDE sur les politiques commerciales, TD/TC/WP(2005)26/FINAL

Fisher, R. et P. Serra (2000), Standards and Protection, Journal of International Economics, 52, 377-400.

Fontagné, L., M. Mimouni et JM Pasteels (2005), Estimating the impact of environmental SPS and TBT on international trade, Integration and Trade Journal 22: 7-37.

Foster, W., et R. Just (1989), Measuring Welfare Effects of Product Contamination with Consumer Uncertainty. Journal of Environmental Economics and Management. 17, 266-283.

Fox, J., D. Hayes, et J. Shogren (2002), Consumer Preferences for Food Irradiation: How Favorable and Unfavorable Descriptions Affect Preferences for Irradiated Pork in Experimental Auctions, Journal of Risk and Uncertainty 24, 1, 75-95.

Freeman, A.M. (1993), Measuring Environmental and Resource Values. Theory and Methods, Resources for the Future, Washington D.C.

Fulponi, L. (2006), Private voluntary standards in the food system: The perspective of major food retailers in OECD countries, Food Policy 31, numéro 1:1-13.

Gandal, N., et O. Shy (2001), Standardization policy and international trade, Journal of International Economics 53(2): 262-282. 
Garcia Martinez, M., A. Fearne, J.A. Caswell, et S. Henson. (2007), Co-regulation as a possible model for food safety governance: Opportunities for public-private partnerships, Food Policy 32: $299-314$

Grothe, U., Deblitz, C. et S. Stegmann (2000), Total costs and environmental standards for selected agricultural products in Brazil, Germany and Indonesia: the impact on international competition, Quarterly Journal of International Agriculture 19(2000), n 3, pp. 299-318.

Hammitt, J. K. (2002). QALYs Versus WTP. Risk Analysis, 22:985-1001.

Harrington, W. et Portney, P.R. (1987), Valuing the Benefits of Health and Safety regulations, Journal of Urban Economics, 22, 1, pp. 101-112.

Hayes, D., J. Shogren, Y. Shin, et J. Kliebenstein (1995), Valuing Food Safety in Experimental Auction Markets, American Journal of Agricultural Economics 77, 40-53.

Henry de Frahan, B., et M. Vancauteren. (2006), Harmonization of food regulations and trade in the Single Market: evidence from disaggregated data, European Review of Agricultural Economics 33(3): 337-360.

Horn, H., et P.C. Mavroidis (2006), « The WTO Dispute Settlement System 1995-2004: Some descriptive statistics », Mimeo.

Huffman W.E, M.C. Rousu, J.F. Shogren, et A. Tegene (2003), The Public Good Value of Information from Agribusinesses on Genetically Modified Food, American Journal of Agricultural Economics 85, 1309-1315.

Huffman W.E, M.C. Rousu, J.F. Shogren, et A. Tegene (2007), The Effects of Prior Beliefs and Learning on Consumers' Acceptance of Genetically Modified Food, Journal of Economic Behavior \& Organization, 63, 193-206.

Hummels, D. (2001), «Time as a trade barrier», mimeo, Purdue University. http://www.krannert.purdue.edu/centers/ciber/publications/pdf/00-007Hummels2.pdf

ITTO (2008), Towards increasing the comparability and acceptance of forest certification systems, ITTO Technical Series $n^{\circ} 29$, by Alan Purbawiyatna and Markku Simula, http://www.itto.or.jp/live/Live_Server/4092/TS29.pdf

Josling T., D. Roberts et D. Orden (2004). Food Regulation and Trade: Toward a Safe and Open Global System. Institute for International Economics, Washington DC, mars 2004.

Jones, R.E., et R.W. Medd. (2000), Economic Thresholds and the Case for Longer Term Approaches to Population Management of Weeds, WeedTech. 14 (avril-juin ): 337-50.

Kee, H. L., A. Nicita. et M. Olarreaga. (2008). «Import Demand Elasticities and Trade Distortions », Review of Economics and Statistics (à paraître).

Kompas, T. et T.N. Che (2003), «A Practical Border Quarantine Measure for Imported Livestock », International and Development Economics Working Papers (03-1), Crawford School of Economics and Government, Australian National University, Canberra.

Kompas, T., Che, T.N., Cao, L.Y. et Klijn, N. (2004), « Optimal Surveillance Measures Against an Imported Pest or Disease: An Application to Papua Fruit Fly in Australia », Australian Bureau of Agricultural and Resource Economics, Canberra.

Kopp, R.J., Krupnick, A.J. et Toman, M. (1997), « Cost Benefit Analysis and Regulatory Reform: An Assessment of the Science and the Art », document de discussion 97-19, Resources for the Future, Washington D.C.

Lagerkvist, C.J., Carlsson, F., et Viske, D. (2006), Swedish consumer preferences for animal welfare and biotech: A choice experiment. AgBioForum, 9(1), 51-58. Disponible à l'adresse http://www.agbioforum.org.

Landelfeld, J.S. et Seskin, E.P. (1982), The Economic Value of Life: Linking Theory and Practice, American Journal of Public Health, 6, 555-66. 
Larson, B. E. (2003), Eco-labels for credence attributes: the case of shade-grown coffee, Environment and Development Economics, 8: 529-547.

Levine, J.M., et C. M. D'Antonio (2003), «Forecasting Biological Invasions with Increasing International Trade », Conservation Biology 17 (1): 322-326.

Levitt, S.D. et List, J.A., 2007. What Do Laboratory Experiments Tell Us About the Real World. Journal of Economic Perspective, 21,153-174.

Lichtenberg, E., D.D. Parker, et D. Zilberman (1988). « Marginal Analysis of Welfare Costs of Environmental Policies: The Case of Pesticide Regulation », American Journal of Agricultural Economics, 70(4):867-74.

List J.A., (2007), Field Experiments: A Bridge between Lab and Naturally Occurring Data. Advances in Economic Analysis \& Policy 6(2), article 8. http://www.bepress.com/bejeap/advances/vol6/iss2/art8 (accès en avril 2007).

Lusk, J.L., Fox J.A. (2003). Value Elicitation in Retail and Laboratory Environments. Economics Letters, 79, 27-34.

Lusk, J.L., Roosen, J., Fox, J.A. (2003). Demand for beef from cattle administered growth hormones or fed genetically modified corn: a comparison of consumers in France, Germany, The United Kingdom and The United States. American Journal of Agricultural Economics 85, 16-29.

Lusk J., J. Brown, T. Mark, I. Proseku, R. Thompson et J. Welsh (2006a). « Public Policy, and Country-of-Origin Labeling », Review of Agricultural Economics, 28(2), 284-292.

Lusk J., W. B. Traill, L. O. House, C. Valli, S. R. Jaeger, M. Moore, B. Morrow (2006b). Comparative Advantage in Demand: Experimental Evidence of Preferences for Genetically Modified Food in the United States and European Union, Journal of Agricultural Economics, 57(1), 1-21.

Lusk, J.L., Shogren J.F (2007a). Experimental Auctions. Methods and Applications in Economic and Marketing Research. Cambridge University Press, Cambridge, Royaume-Uni.

Lusk, J.L., Shogren J.F (2007b), Chapitre 1. Introduction. in Experimental Auctions. Methods and Applications in Economic and Marketing Research. Cambridge University Press, Cambridge, Royaume-Uni. http://www.cambridge.org/catalogue/catalogue.asp?isbn=9780521671248\&ss=exc ～(accès disponible en juillet 2008).

Maertens, M., et J.F.M. Swinnen. (2009). «Trade, Standards, and Poverty: Evidence from Senegal », World Development 37, numéro 1, janvier 2009, pages 161-178

Maertens, M., L. Dries, F.A. Dedehouanou, et J.F.M. Swinnen. 2007. « High-value supply chains, food standards and rural households in developing countries ». dans J.F.M. Swinnen, dir. pub.. Global supply chains, standards and the poor. Cabi Publishing, Wallingford.

Marette S. (2007) Minimum Safety Standard, Consumer's Information and Competition, Journal of Regulatory Economics, 32, 259-285.

Marette, S., et J.C. Beghin (2007), « Are Standards Always Protectionist? » CARD Working Paper \#07-WP 450, juin.

Marette S. (2008). Standards and Labels, Economics Bulletin, 12, 2 (2008) 1-7.

Marette S., J. Roosen, S. Blanchemanche, P. Verger (2008a). The Choice of Fish Species: An Experiment Measuring the Impact of Risk and Benefit Information. Journal of Agricultural and Resource Economics, 33, 1-18.

Marette S., J. Roosen, S. Blanchemanche (2008b). Taxes and Subsidies to Change Eating Habits when Information is not enough: An Application to Fish Consumption. Journal of Regulatory Economics, 34, 119-143. 
Marette S., J. Roosen, S. Blanchemanche (2008c). Health Information and Substitution between Fish: Lessons from Laboratory and Field Experiments. Food Policy, 33, 197-208.

Marra, M.C., et Piggott, N.E. (2006). The value of non-pecuniary characteristics of crop biotechnologies: A new look at the evidence. In Just, Alston, and Zilberman (dir. pub.), Regulating Agricultural Biotechnology: Economics and Policy (pp. 145-178). New York, éditions Springer-Verlag.

Maskus, K., Otsuki, T. et J.S. Wilson (2005), The costs of compliance with product standards for firms in developing countries: an econometric study, document de travail $n^{\circ} 3590$, Banque mondiale, Washington DC.

MAST (Équipe d'appui multiorganisations sur les ONT), (2008), First Progress Report to the Group of Eminent Persons on Non-tariff Barriers, (juin 2008). Mimeo, Genève, CNUCED.

Moenius, J. 1999. «Information versus Product Adaptation: The role of Standards in Trade », document de travail, University of California, San Diego.

2006. «The Good, the Bad and the Ambiguous: Standards and Trade in Agricultural Products », article présenté lors du colloque d'été de l'IATRC sur le thème « Food Regulation and Trade: Institutional Framework, Concepts of Analysis and Empirical Evidence », à Bonn en Allemagne, du 28 au 30 mai.

Melitz, M. (2003), The Impact of Trade on Intra-Industry Reallocations and Aggregate Industry Productivity, Econometrica, 71(6), pp.1695 - 1725.

Morall J. (2003), Saving Lives: A review of the Record. Journal of Risk and Uncertainty, 27: 221237.

Napolitano F., C. Pacelli, A. Girolami et A. Braghieri (2008), Effect of Information About Animal Welfare on Consumer Willingness to Pay for Yogurt, Journal of Dairy Science. 91:910-917

Noussair, C., S. Robin, et B. Ruffieux (2002), Do Consumers Not Care about Biotech Foods or Do They Just Not Read the Labels? Economics Letters 75: 47-53.

Nimon, W. et J. Beghin (1999), Are Eco-Labels Valuable? Evidence From The Apparel Industry, American Journal of Agricultural Economics 81(3): 801-811.

Polinsky A. M., Rogerson, W. (1983). Products Liability and Consumer Misperceptions and Market Power. The Bell Journal of Economics 14, 581-89.

OCDE (2002a), L'agriculture et la libéralisation des échanges: Élargir la portée des Accords d'Uruguay. Paris: Les éditions de l'OCDE.

OCDE (2002b), Autres scénarios de libéralisation et leurs incidences sur les rentes de contingent et les recettes tarifaires dans quelques pays de l'OCDE. COM/AGR/TD/WP(2002)23/FINAL 19 novembre 2002.

OCDE (2006a), Rapport final sur les normes privées et l'évolution de la filière agroalimentaire, Groupe de travail des politiques et des marchés agricoles. AGR/CA/APM(2006)9/FINAL.

OCDE (2006b), Les normes privées et l'accès des pays en développement aux chaînes de valeur mondiales : enjeux et perspectives, conclusions de quatre études de cas, Groupe de travail des politiques et des marchés agricoles (Octobre 2006), AGR/CA/APM(2006)20.

OCDE (2006c), Analyse coûts-bénéfices et environnement: Développements récents, (auteurs : David Pearce, Giles Atkinson et Susana Mourato), Paris: Les Éditions de l’OCDE.

OCDE (2007a), Scoping paper: economic assessment of non-tariff measures in agro-food trade, Groupe de travail mixte sur l'agriculture et les échanges (octobre 2007). TAD/TC/CA/WP(2007)3.

OCDE (2007b), Documentation of the AGLINK_COSIMO model, Groupe de travail des politiques et des marchés agricoles, AGR/CA/APM(2006)16/FINAL 
OCDE (2008a), Interactions entre normes publiques et normes privées dans la filière alimentaire, Groupe de travail des politiques et des marchés agricoles, May 2008. AGR/CA/APM(2006)21/REV2.

OCDE (2008b), Analyse des méthodes quantitatives d'évaluation de l'impact des normes sur les échanges dans le secteur agroalimentaire, Groupe de travail mixte sur l'agriculture et les échanges (juin 2008), TAD/TC/CA/WP(2007)1/REV1.

OCDE (2008c), Identifying trade facilitating and trade limiting non-tariff measures - what does the data tell us? Groupe de travail mixte sur l'agriculture et les échanges (octobre 2008) (TAD/TC/CA/WP(2008)5.

OFIMER (2007). Office National Interprofessionnel des Produits de la Mer et de L'Aquaculture. Commerce Extérieur des Produits de la Pêche et de l'Aquaculture. Bilan Annuel 2007. Paris.

Orden, D., et E. Romano (1996). «The Avocado Dispute and Other Technical Barriers to Agricultural Trade Under NAFTA », contribution à la conférence « NAFTA and Agriculture: Is the Experiment Working », San Antonio, Texas, novembre.

Otsuki, T., J.S. Wilson, et M. Sewadeh (2001), What Price Precaution? European Harmonisation of Aflatoxin Regulations and African Groundnut Exports, European Review of Agricultural Economics 28(3):263-283.

Paarlberg, P.L., et J.G. Lee. (1998), Import restrictions in the presence of a health risk: an illustration using FMD. American Journal of Agricultural Economics Vol. 80(1): 175-183.

Pendell, D.L., J. Leatherman, T.C. Schroeder, et G.S. Alward. (2007), The Economic Impacts of a Foot-And-Mouth Disease Outbreak: A Regional Analysis, Journal of Agricultural and Applied Economics 39 (octobre), 19-33.

Peterson, E.B., et D. Orden. (2008), Avocado Pests and Avocado Trade, American Journal of Agricultural Economics, 90: 321-335.

Piggott, N.E., et M.C. Marra. (2007).,The Net Gain to Cotton Farmers of a Natural Refuge Plan for Bollgard II ${ }^{\circ}$ Cotton, AgBioForum, 10(1): 1-10.

Pimentel, D., R. Zuniga, et D. Morrison. (2005). «Update on the environmental and economic costs associated with alien-invasive species in the United States », Ecological Economics 52 : $273-288$.

Pimentel, D., L. Lach, R. Zuniga, et D. Morrison (2000). « Environmental and Economic Costs of Nonindigenous Species in the United States », Biosci. 50 (janvier): 53-65.

Pollak, R.A. (1995), Regulating risks, Journal of Economic Literature, mars, 33, 1, pp 179-91.

Pollak, R.A. (1998). Imagined risks and cost-benefit analysis, American Economic Review, Papers and Proceedings, 88, 2, pp 376-79.

Qaim, M., et I. Matuschke. (2005). Impacts of genetically modified crops in developing countries: a survey, Quarterly Journal of International Agriculture Vol. 44 n 3: 207-227

Rau M. L, et F. van Tongeren. (2007). Modeling differentiated quality standards in the agri-food sector: the case of meat trade in the enlarged EU. Agricultural Economics, 37: 305-315.

Rau M. L, et F. van Tongeren. (2008). « Heterogeneous firms and homogenising standards in agrifood trade- the Polish meat case ». étude réalisée pour la conférence de l'European Trade Study Group, septembre 2008.

Reardon, T., Timmer, C.P., Barrett, C. et J. Berdegué (2003), The Rise of Supermarkets in Africa, Asia, and Latin America, American Journal Agricultural Economics 85(5): 1140-1146.

Rozan, A., Stenger, A., Willinger, M., (2004). Willingness-to-Pay for Food Safety: An Experimental Investigation of Quality Certification on Bidding Behaviour. European Review of Agricultural Economics, 31(4), 409-425. 
Rousu, M.C., W.E. Huffman, J.F. Shogren, et A. Tegene (2004). «Estimating the Public Value of Conflicting Information: The Case of Genetically Modified Foods », Land Economics 80(1): 125-135.

Rousu, M.C., W.E. Huffman, J.F. Shogren, et A. Tegene (2007). « Effects and Value of Verifiable Information in a controversial market: Evidence from Lab Auctions of Genetically Modified Food », Economic Inquiry, 45(3): 409-432.

Shogren J.F., Fox, J.A., Hayes, D.J., Roosen, J. (1999). Observed Choices for Food Safety in Retail, Survey, and Auction Markets. American Journal of Agricultural Economics 81(5), 1192-1199.

Shogren J. (2006). Valuation in the Lab. Environmental \& Resource Economics 34, 163-172.

Sobolevsky A., G. Moschini et H. Lapan (2005). «Genetically Modified Crops and Product differentiation: Trade and Welfare Effects in the Soybean Complex », American Journal of Agricultural Economics 83(1): 133-149.

Stoneham, G., V.Chaudhri,, A.Ha et L. Strappazzon (2003), Auctions for conservation contracts: an empirical examination of Victoria's BushTender trial, Australian Journal of Agricultural and Resource Economics. Vol. 47 (4): 477-500.

Sutton, J. (1991), Sunk Costs and Market Structure: Price Competition, Advertising, and the Evolution of Concentration. MIT Press, Cambridge, Massachusetts.

Swinton, S.M., D.D. Buhler, F. Forcella, J.L. Gunsolus, et R.P. King. (1994). « Estimation of Crop Yield Loss Due to Interference by Multiple Weed Species », Weed Science Vol. 42 (1): 103109.

Taylor, C.R., et O.R. Burt. « Near-optimal Management Strategies for Controlling Wild Oats in Spring Wheat », Amer. J. Agr. Econ. 66 (février 1984):50-60.

Teisl, M., N. Bockstael, et A. Levy (2001). «Measuring the Welfare Effects of Nutrition Information », American Journal of Agricultural Economics 83(1): 133-149.

Teisl M., B. Roe et R. Hicks. 2002. Can Eco-labels Tune a Market? Evidence from Dolphin-Safe Labelling. Journal of Environmental Economics and Management. 43:339-359

Wansink B., S. Sonka et C. Hasler (2004). Front-Label Health Claims: When Less is More. Food Policy, 29: 659-667.

Wilson, J.S., et T. Otsuki. 2004b. « Standards and Technical Regulations and Firms in Developing Countries: New Evidence from A World Bank Technical Barriers to Trade Survey », Banque mondiale, juin, version provisoire.

Wilson N.L. et J. Anton (2006). Combining risk Assessment and Economics in Managing a Sanitary-Phytosanitary Risk. American Journal of Agricultural Economics, 88, 194 - 202.

Wolfenbarger, L.L., et P.R. Phifer. (2000). «The Ecological Risks and Benefits of Genetically Engineered Plants », Science 290: 2088-93.

Yue, C., Beghin, J.C. et H.H. Jenson (2006), « Tariff equivalent of technical barriers to trade with imperfect substitution and trade costs », American Journal of Agricultural Economics, 88(4), pp. 947-960. 


\title{
Annexe 1
}

\section{Classification des mesures non tarifaires établie par la MAST}

\author{
Classification des mesures non tarifaires (version de juin 2008)
}

A000 MESURES SANITAIRES ET
PHYTOSANITAIRES

A100 Normes volontaires

\section{A110 Normes internationales}

A111 Normes relatives aux processus de
production

A112 Normes relatives aux caractéristiques du produit

A119 Normes internationales, n.d.a.

\section{A120 Normes nationales}

\begin{abstract}
A121 Normes relatives aux processus de production
\end{abstract}

A122 Normes relatives aux caractéristiques du produit

A129 Normes nationales, n.d.a.

\section{A130 Normes régionales}

\author{
A131 Normes relatives aux processus de \\ production \\ A132 Normes relatives aux caractéristiques du \\ produit
}

Les mesures sanitaires et phytosanitaires comprennent les lois, décrets, règlements, normes et procédures destinés à protéger la vie ou la santé humaine, animale ou végétale. Règles, principes directeurs ou caractéristiques de produits ou de processus et de méthodes de production conçus pour protéger la santé ou la vie humaine, animale ou végétale (concernant par exemple des compositions, la qualité ou l'hygiène), approuvés par des organismes reconnus à l'échelon régional, national ou international, ou bien établies par des entreprises privées. Les normes volontaires $n$ 'ont pas force de loi.

Normes mises au point par des agences de normalisation internationales. Par définition, les normes internationales sont adaptées à un usage mondial.

Normes définissant des processus de la chaîne de production censés contribuer à la sécurité et à la qualité des produits.

Normes définissant les caractéristiques requises pour certains produits (telles que la taille, la couleur, la composition et la qualité) et contribuant à la sécurité et la qualité du produit.

De manière générale, chaque pays ou économie compte une seule agence de normalisation officielle. L'Accord SPS autorise les pays à mettre en place leurs propres normes, qui doivent s'appuyer sur des éléments scientifiques. L'application de ces règles doit se limiter au champ nécessaire à la protection de la vie ou de la santé humaine, animale ou végétale. Ces règles ne doivent pas être employées de manière arbitraire ou injustifiable à des fins discriminatoires entre les pays où existent des conditions identiques ou similaires.

Normes définissant des processus de production contribuant à la sécurité et à la qualité des produits.

Normes régissant les caractéristiques requises des produits (telles que la taille, la couleur, la composition ou la qualité), censées contribuer à la sécurité et à la qualité de ceux-ci.

Normes imposées par un État ou par une région d'un pays et exigeant des caractéristiques supplémentaires au-delà des normes nationales.

Normes définissant les processus de production censés contribuer à la sécurité et à la qualité des produits.

Normes régissant les caractéristiques requises des produits (telles que la taille, la couleur, la composition ou la qualité), censées contribuer à la sécurité et à la qualité de ceux-ci. 
A139 Normes régionales, n.d.a.

A140 Normes privées*

A200 Réglementation sanitaire et phytosanitaire

A210 Réglementation en matière d'étiquetage, de marquage et de conditionnement

A211 Règlementation en matière d'étiquetage

A212 Règlementation en matière de marquage

A213 Règlementation en matière de conditionnement

A220 Règlementation en matière de traçabilité

A221 Origine des matières premières et produits semi-finis

A222 Historique de transformation

A223 Distribution et emplacement des produits après livraison

A229 Réglementation en matière de traçabilité, n.d.a.

A230 Limites de tolérance pour les résidus et contaminants, ou restriction de l'usage de certaines substances

A231 Limites de tolérance pour les résidus de, ou de contamination par certaines substances dans les produits alimentaires et le fourrage
Normes exigées par des entités privées, telles que des instances représentatives de chaînes de supermarchés ou d'autres organismes (par ex. réglementation sur les pesticides, traçabilité et hygiène générale des produits alimentaires).

La réglementation sanitaire et phytosanitaire comprend des lois, décrets, exigences et procédures destinées à protéger la vie ou la santé humaine, animale ou végétale. Son respect a un caractère obligatoire.

Mesures de réglementation de la catégorie, de la couleur et de la taille du texte sur les emballages et étiquettes des produits de consommation et de définition de l'information directement liée à la sécurité alimentaire qu'il serait possible ou souhaitable de fournir au consommateur. L'étiquetage désigne toute mention écrite, électronique ou graphique portée sur l'emballage destiné au consommateur ou sur une étiquette distincte, mais attachée au produit.

Mesures définissant les informations à des fins de transport et de passage en douane qui devront figurer sur le conditionnement des biens lors de leur transport/distribution, et directement liées à la sécurité alimentaire.

Mesures de réglementation du mode de conditionnement des produits recommandé ou contre-indiqué, conformément à l'équipement de manutention du pays importateur ou pour d'autres raisons, et définissant les matériaux d'emballage à employer, directement liées à la sécurité alimentaire.

La traçabilité correspond à la communication d'informations sur l'origine des animaux vivants et des produits animaux, ainsi que des produits agricoles, y compris leur processus de transformation, mais aussi leur distribution et leur emplacement après livraison. La traçabilité vise à suivre le produit à travers toutes ses phases de production et de distribution.

Description de l'origine géographique des animaux, des plantes et de leurs produits dérivés.

Description de tous les stades de la production Description du transport, de la manutention et du stockage des produits une fois le processus de production achevé.

Concentration maximale autorisée de résidus (LMR) dans les produits alimentaires, le fourrage, le bois, les plantes, etc. ou restriction de l'usage de certaines substances comme ingrédients.

Limite maximale autorisée (LMR) de substances et contaminants toxiques et autrement nuisibles intégrés au produit au cours de la production et/ou de la distribution (tels qu'insecticides, métaux lourds, polluants organiques persistants et produits chimiques dégagés en cours de traitement, comme l'asacryl amide). 
A232 Restriction de l'usage de certaines substances dans les produits alimentaires et le fourrage

A240 Réglementation applicable aux produits alimentaires ou fourrages issus de, ou produits à l'aide d'organismes génétiquement modifiés (OGM)

A250 Exigences en matière d'hygiène

A260 Mesures de prévention des maladies A261 Restriction/interdiction en cas d'épidémie

A262 Obligation de quarantaine

A270 Réglementation des processus de production

A271 Processus de croissance végétale A272 Processus d'élevage ou de capture d'animaux

A273 Traitement des produits alimentaires et du fourrage, y compris le stockage et le transport A279 Réglementation des processus de production, n.d.a.

A280 Restrictions géographiques

A290 Réglementation sanitaire et phytosanitaire, n.d.a.

A300 Évaluation de la conformité avec les SPS
Restriction de l'usage de certaines substances utilisées comme ingrédients, et raisonnablement supposées se retrouver dans le produit final. En conséquence, leur mention est également importante pour prévenir les risques liés à leur emploi.

Cette réglementation peut englober l'étiquetage et les obligations d'autorisation ou d'interdiction pure et simple.

Restrictions visant à éviter la contamination des produits alimentaires et du fourrage par des micro-organismes et des parasites, au stade de la production, de la fabrication, $d u$ transport et du stockage. Comprend les traitements après récolte et le contrôle des pathogènes.

Mesures de protection des animaux, des humains et des plantes contre toute pathologie infectieuse/contagieuse. Englobe les restrictions autres que la quarantaine. Les mesures incluses dans cette catégorie sont généralement prises pour des besoins spécifiques et limitées dans le temps. Obligation d'observation d'une quarantaine des importations durant une période donnée. Il ne s'agit pas d'une mesure prohibitive. La quarantaine s'accompagne de frais de quarantaine, d'inspection, de vétérinaire, de chargement, de fumigation, etc.

Cette appellation générique recouvre la réglementation relative à la sécurité liée au processus de production (dont l'HACCP). Elle concerne principalement la production (plantes et animaux) et la transformation. Les réglementations nationales prévoyant que seuls les produits alimentaires produits dans le respect de certaines pratiques codifiées seront acceptés à la vente sont également incluses dans cette catégorie.

Interdiction d'importation de produits spécifiques en provenance de pays ou de régions donnés en raison de la présence de risques phytosanitaires (tels que des insectes, des acariens ou des pathogènes végétaux).

Procédures de contrôle, d'inspection et d'approbation, y compris concernant le prélèvement d'échantillons, les essais et l'inspection, l'évaluation, la vérification et la garantie de conformité, ainsi que les autorisations et agréments. 


\begin{tabular}{l}
\hline A310 Obligation de certification \\
\hline A311 Certification par les agences \\
gouvernementales du pays d'origine \\
A312 Certification par des agences locales du \\
marché cible \\
A320 Non-acceptation des certifications \\
A321 Non-acceptation des organes \\
internationaux officiels de certification \\
A322 Non-acceptation des certificats des \\
organismes d'évaluation de la conformité émis \\
dans le pays d'origine
\end{tabular}

A323 Refus de l'autodéclaration de conformité

A329 Non-acceptation des certifications, n.d.a.

A330 Réglementation en matière d'essais

A340 Obligation d'inspection et d'approbation
Obligation de certification, dans le pays importateur ou exportateur.

Obligation d'obtenir des certifications de la part du pays exportateur.

Obligation d'obtenir des certifications de la part du pays importateur.

Les certifications émises par un pays ou un organisme donnés ne sont pas reconnues par le pays importateur. Le pays importateur ne reconnaît pas les certifications internationales.

Le pays importateur ne reconnaît pas les certifications de l'exportateur.

Autodéclaration de conformité : procédure en vertu de laquelle un fournisseur atteste par écrit la conformité d'un produit à une réglementation donnée.

Comprend la réglementation en matière de prélèvement d'échantillons, généralement associée aux frais de test ou de laboratoire.

Une inspection et/ou une approbation des importations est obligatoire. L'inspection peut être effectuée par un organisme public ou privé.

Les importateurs peuvent devoir s'enregistrer dans leur pays; tel est souvent le cas pour les produits sensibles tels que les médicaments et/ou les stupéfiants. Les exportateurs doivent contacter un importateur immatriculé.

Pour une réglementation donnée, le même essai doit être effectué dans le pays cible à des points d'entrée nationaux, régionaux et/ou locaux.

Certains documents doivent être traduits dans la langue des pays importateurs.

Certains biens doivent recevoir un agrément pour passer un point d'entrée spécifique du pays importateur à des fins d'essais ou d'inspection.

A390 Évaluation de la conformité avec les SPS, n.d.a.

A900 Mesures sanitaires et phytosanitaires,

n.d.a.

\section{B000 OBSTACLES TECHNIQUES AU COMMERCE}

B100 Normes volontaires

\footnotetext{
Les obstacles techniques au commerce (OTC) sont des réglementations/normes concernant la spécification technique des produits et les systèmes d'évaluation de la conformité de ces derniers.

Règles, principes directeurs ou caractéristiques de produits ou de processus et de méthodes de production conçus pour protéger la santé ou la vie humaine, animale ou végétale (concernant par exemple des compositions, la qualité ou hygiène), approuvés par des organismes reconnus à l'échelon régional, national ou international, ou bien établies par des entreprises privées. Les normes volontaires n'ont pas force de loi.
} 


\section{B110 Normes internationales}

B111 Normes relatives aux processus de production

B112 Normes relatives aux caractéristiques du produit

B113 Normes relatives au système de gestion

B119 Normes internationales, n.d.a.

\section{B120 Normes nationales}

\section{B121 Normes relatives aux processus de production}

B122 Normes relatives aux caractéristiques du produit

B129 Normes nationales, n.d.a.

B130 Normes régionales

B131 Normes relatives aux processus de production

B132 Normes relatives aux caractéristiques du produit

B139 Normes régionales, n.d.a. B140 Normes privées*

B200 Réglementation technique

B210 Réglementation en matière d'étiquetage, de marquage et de conditionnement B211 Réglementation en matière d'étiquetage
Normes mises au point par des agences de normalisation internationales. Par définition, les normes internationales sont adaptées à un usage mondial.

Normes définissant des processus de la chaîne de production censés contribuer à la sécurité et à la qualité des produits.

Normes définissant les caractéristiques requises pour certains produits (telles que la taille, la couleur, la composition et la qualité) et contribuant à la sécurité et la qualité du produit. Englobe également les caractéristiques de performance du produit.

Normes fixant des exigences ou fournissant des recommandations de bonnes pratiques de gestion et établissant un cadre de production (par ex., le système de qualité d'une entreprise manufacturière peut englober la recherche de processus de fabrication plus efficaces ou l'accélération de la distribution).

Normes techniques applicables au niveau national et réglementant les caractéristiques techniques des produits, ainsi que les processus de production.

Normes définissant des processus de production contribuant à la sécurité et à la qualité des produits.

Normes régissant les caractéristiques requises des produits (telles que la taille, la couleur, la composition ou la qualité), censées contribuer à la sécurité et à la qualité de ceux-ci. Concernent également les performances du produit.

Normes imposées par un État ou par une région d'un pays et exigeant des caractéristiques supplémentaires au-delà des normes nationales.

Normes définissant les processus de production censés contribuer à la sécurité et à la qualité des produits.

Normes régissant les caractéristiques requises des produits (telles que la taille, la couleur, la composition ou la qualité), censées contribuer à la sécurité et à la qualité de ceux-ci. Concernent également la performance des produits. Normes exigées par des organismes non gouvernementaux, tels que des entreprises privées (par ex. les normes applicables aux logiciels ou aux appareils électriques).

Mesures de réglementation de la catégorie, de la couleur et de la taille du texte sur les emballages et étiquettes des produits de consommation et de définition de l'information qu'il serait possible ou souhaitable de fournir au consommateur. L'étiquetage désigne toute mention écrite, électronique ou graphique portée sur l'emballage destiné au consommateur ou sur une étiquette distincte, mais attachée au produit. 
B212 Réglementation en matière de marquage conditionnement

B220 Réglementation en matière de traçabilité

B221 Origine des matières premières et des produits semi-finis

B222 Historique de transformation

B223 Distribution et emplacement des produits après livraison

B229 Réglementation en matière de traçabilité, n.d.a.

B230 Limites de tolérance pour les résidus ou restriction de l'usage de certaines substances B231 Limites de tolérance pour les résidus ou de contamination par certaines substances

B232 Restriction de l'usage de certaines substances

B240 Réglementation relative aux organismes génétiquement modifiés (pour d'autres raisons que la sécurité alimentaire)

B250 Exigences d'identification

B260 Contraintes visant spécifiquement à protéger l'environnement

B270 Autres exigences relatives aux

caractéristiques du produit

B280 Autres exigences relatives au processus de

production

B290 Réglementation technique, n.d.a.

B300 Évaluation de la conformité relativement aux OTC
Mesures définissant les informations à des fins de transport

et de passage en douane qui devront figurer sur le

conditionnement des biens lors de leur

transport/distribution.

Mesures de réglementation du mode de conditionnement des produits recommandé ou contre-indiqué, conformément à l'équipement de manutention du pays importateur ou pour d'autres raisons, et définissant les matériaux d'emballage à employer.

La traçabilité correspond à la communication d'informations sur l'origine des matières premières et des produits semi-finis, y compris le déroulement de la transformation de ceux-ci, mais aussi leur distribution et leur emplacement après livraison. La traçabilité vise à suivre le produit à travers toutes ses phases de production et de distribution.

Description de l'origine géographique des matières premières et des produits semi-finis.

Description de tous les stades de la production

Description du transport, de la manutention et du stockage des produits une fois le processus de production achevé. Concentration maximum ou restriction de l'usage de certaines substances.

Limites maximales des substances et contaminants toxiques et autrement dangereux intégrés au produits lors $d u$ processus de production.

Restriction d'usage de certaines substances en tant que composants ou additifs, et dont on peut raisonnablement penser qu'elles se retrouveront dans le produit fini. De ce fait, leur identification est également importante pour prévenir les risques liés à leur usage.

Restriction des importations en cas d'utilisation

d'organismes génétiquement modifiés au cours de la production.

Conditions à respecter pour l'identification d'un produit portant une dénomination donnée (telles que la concentration minimum en pourcentage d'un ingrédient qui donne son nom au produit final, par exemple le cacao dans le chocolat).

Conditions ou exigences visant à prévenir les dommages écologiques ou à assurer la protection de l'environnement.

Procédures de contrôle, d'inspection et d'approbation, y compris concernant le prélèvement d'échantillons, les essais et l'inspection, l'évaluation, la vérification et la garantie de conformité, ainsi que les autorisations et agréments. 
B310 Obligation de certification

B311 Certification par les agences gouvernementales du pays d'origine B312 Certification par les agences locales sur le marché cible

B320 Non-acceptation des certifications

B321 Non-acceptation des organismes internationaux officiels d'évaluation de la conformité

B322 Non-acceptation des certificats des organismes d'évaluation de la conformité émis dans le pays d'origine

B323 Refus de l'autodéclaration de conformité

B329 Non-acceptation, n.d.a.

B330 Réglementation en matière d'essais

B340 Réglementation en matière d'inspection et d'approbation

B350 Obligation d'enregistrement

B360 Répétition sur le marché cible d'essais identiques pour une réglementation identique ou équivalente

B370 Obligation de traduction de rapports ou de certificats

B380 Obligation de passage à certains points d'entrée ou postes douaniers spécifiques

B390 Évaluation de la conformité liée aux OTC, n.d.a.

B900 Obstacles techniques au commerce, n.d.a.

\section{C000 AUTRES MESURES TECHNIQUES}

C100 Inspection préalable à la livraison

C200 Formalités douanières particulières sans rapport avec les SPS/OTC

C210 Obligation de documentation

C220 Obligation d'expédition directe

c220 Obligation d'expédition directe
Obligation de certification, dans le pays importateur ou

exportateur.

Obligation d'obtenir des certifications de la part du pays

exportateur.

Obligation d'obtenir des certifications de la part du pays importateur.

Les certifications émises par un pays ou un organisme donnés ne sont pas reconnues par le pays importateur.

Le pays importateur ne reconnaît pas les certifications internationales.

Le pays importateur ne reconnaît pas les certifications de l'exportateur.

Autodéclaration de conformité : procédure en vertu de laquelle un fournisseur atteste par écrit la conformité d'un produit à une réglementation donnée.

Comprend la réglementation en matière de prélèvement d'échantillons, généralement associée aux frais de test ou de laboratoire.

Une inspection et/ou une approbation des importations est obligatoire. L'inspection peut être effectuée par un organisme public ou privé.

Les importateurs peuvent devoir s'enregistrer dans leur pays ; tel est souvent le cas pour les produits sensibles qui peuvent susciter des problèmes de sécurité. Les exportateurs doivent contacter un importateur immatriculé.

Pour une réglementation donnée, le même essai doit être effectué dans le pays cible à des points d'entrée nationaux, régionaux et/ou locaux.

Certains documents doivent être traduits dans la langue des pays importateurs.

Certains biens doivent recevoir un agrément pour passer un point d'entrée spécifique du pays importateur à des fins d'essais ou d'inspection. 


\author{
C230 Obligation de passage à certains postes \\ douaniers spécifiques
}

C240 Restrictions de transport

\section{C241 Réglementation restrictive sur le transport} aérien

C242 Réglementation restrictive sur le transport maritime

C243 Réglementation restrictive sur le transport terrestre

C290 Formalités douanières particulières sans rapport avec les SPS/OTC, n.d.a.

C900 Mesures techniques, n.d.a. D000 MESURES DE CONTRÔLE DES PRIX

D100 Fixation administrative des prix

D110 Prix minimum des importations

D120 Prix de référence et autres mesures de contrôle des prix

D190 Fixation administrative des prix, n.d.a.
D200 Limitation volontaire des prix à l'exportation

D300 Charges variables
Les biens doivent passer par un point d'entrée et/ou un poste douanier spécifique, ce qui peut ralentir le processus d'autorisation des importations.

Conditions de transport, normes ou lois spécifiques exigées par les autorités nationales de chaque pays, et susceptibles d'être considérées comme restrictives.
Les mesures de contrôle des prix sont mises en ouvre afin de contrôler les prix des articles importés, pour les raisons suivantes: subventionner les prix des biens produits dans le pays, si les mêmes biens importés sont moins chers ; fixer le prix domestique de certains produits en raison de la fluctuation des prix des marchés intérieurs, ou de l'instabilité des prix d'un marché étranger ; pallier les préjudices résultant de pratiques commerciales étrangères "déloyales».

La fixation administrative des prix consiste, pour les autorités du pays importateur, à prendre en compte les prix du producteur ou du consommateur sur le marché intérieur, à fixer des limites de prix à la hausse et à la baisse, ou à s'en remettre à des valeurs de marché internationales déterminées. Il peut exister différentes méthodes de fixation des prix, telles que la mise en place de prix minimum des importations ou de fixation des prix en fonction d'un niveau de référence.

Prix à l'importation déterminé d'avance et en dessous duquel l'importation ne peut avoir lieu.

Prix à l'importation déterminé d'avance, utilisé comme référence par les autorités du pays importateur pour la fixation d'un prix minimum ou maximum.

Une limitation volontaire des prix à l'exportation est un accord selon lequel l'exportateur accepte de maintenir le prix de ses produits au-dessus d'un certain seuil.

Les charges variables sont des taxes ou des droits visant à aligner le prix des produits agricoles et alimentaires importés sur ceux des prix des produits intérieurs correspondants. Les charges sur les matières premières peuvent être calculées en fonction du poids total, tandis que celles sur les produits alimentaires transformés dépendront de la proportion de matière première dans le produit fini. Ces charges se décomposent comme suit : 
D310 Taxes variables

D390 Charges variables, n.d.a.

D320 Composantes variables
Le taux de ces taxes est inversement proportionnel au prix des importations. Ces charges concernent principalement les produits primaires. Elles peuvent également être désignées sous l'appellation de prélèvements variables à l'importation.

La taxe comprend une composante fixe et une composante variable. Ces charges sont principalement appliquées aux produits transformés, tandis que la part variable concerne les produits primaires ou les ingrédients qui entrent dans la composition du produit fini. Ces composantes peuvent également être désignées sous l'appellation d'éléments compensatoires.

\section{D400 Mesures antidumping}

\section{D410 Enquêtes antidumping}

\section{D420 Droits antidumping}

\section{D430 Engagements de prix antidumping}

Les mesures antidumping visent à contrer une pratique de dumping de la part d'un exportateur. On considère qu'il y a dumping lorsqu'un produit est introduit sur le marché d'un pays importateur à un prix inférieur à sa valeur normale, c'est-à-dire lorsque le prix d'un produit exporté est inférieur au prix comparable, dans des conditions commerciales ordinaires, du même produit destiné à la consommation intérieure du pays exportateur.

Les enquêtes antidumping ont lieu soit suite à une plainte des producteurs locaux de biens similaires, soit de la propre initiative des autorités du pays importateur, qui ont des raisons de penser que cette pratique peut porter un lourd préjudice aux producteurs nationaux concurrents ou aux exportateurs de tierces parties. Il est possible de prélever des droits provisoires pendant l'enquête.

Les droits antidumping sont prélevés sur certains biens provenant de partenaires commerciaux spécifiques, afin de compenser la marge de dumping. Le montant de ces droits dépend généralement de l'entreprise concernée.

Les exportateurs peuvent proposer de relever leurs prix à l'exportation pour éviter de se voir imposer des droits antidumping. Selon les principes de l'OMC, les prix peuvent faire l'objet de négociations à cette fin, mais seulement après que le dumping aura été prouvé.

\section{D500 Mesures compensatoires}

Les mesures compensatoires visent à compenser toute subvention directe ou indirecte accordée par les autorités du pays exportateur. Elles peuvent prendre la forme de droits compensatoires ou d'engagements de prix de la part des entreprises exportatrices ou des autorités du pays qui a accordé les subventions. 


\begin{tabular}{ll} 
D510 Enquêtes compensatoires & Les enquêtes compensatoires sont lancées soit à la suite \\
& d'une plainte des producteurs locaux de biens similaires, \\
& soit sur l'initiative de l'autorité du pays importateur, afin de \\
déterminer si les biens importés bénéficient de subventions & et s'ils peuvent causer un préjudice grave. \\
\hline D520 Droits compensatoires & Droits prélevés sur certains biens afin de compenser le \\
& montant de la subvention accordée par l'exportateur sur le \\
produit concerné, si cette subvention est supposée affecter & l'économie nationale. \\
Les exportateurs peuvent proposer de s'engager à relever & les prix à l'exportation pour éviter de se voir imposer des \\
droits compensatoires. Selon les règles de l'OMC, les prix & peuvent faire l'objet de négociations à cette fin, mais \\
seulement après que l'effet préjudiciable de la subvention \\
aura été prouvé. \\
Droits d'urgence etlou temporaires, imposés dans le cadre \\
d'une action de sauvegarde. Un pays peut engager de telles \\
mesures (c'est-à-dire restreindre temporairement les \\
importations d'un produit) afin de protéger un secteur \\
national donné d'une augmentation des importations d'un \\
produit susceptible de causer, ou causant déjà, de graves \\
préjudices au secteur national producteur de biens \\
similaires ou en concurrence directe avec ce produit. \\
Des droits saisonniers sont applicables à certaines périodes \\
de l'année, habituellement en rapport avec les produits \\
agricoles.
\end{tabular}

D900 Mesures de contrôle des prix, n.d.a.

EO00 MESURES DE CONTRÔLE DE LA QUANTITÉ

\section{E100 Licence non automatique}

E110 Licence sans critère préalable particulier

E120 Licence à usage spécifique

E130 Licence liée à une production locale

\author{
Les mesures de contrôle de la quantité visent à limiter la \\ quantité de biens pouvant être importés, qu'ils proviennent \\ d'un seul fournisseur ou de plusieurs. Ces mesures peuvent \\ prendre la forme de licences restrictives, de la mise en place \\ de contingents prédéterminés ou d'interdictions. \\ (La plupart des mesures du contrôle de la quantité sont \\ formellement prohibées par les accords du GATT de 1994, \\ mais elles peuvent être appliquées dans certaines \\ circonstances bien définies (article XI)) \\ Cette licence est une licence d'importation qui n'est pas \\ accordée automatiquement. Elle peut être octroyée soit sur \\ une base discrétionnaire, soit en fonction de certains \\ critères spécifiques avant sa délivrance. \\ Cette licence, quelquefois aussi qualifiée de licence \\ discrétionnaire, est émise à la discrétion de l'autorité \\ responsable. \\ Cette licence est limitée aux opérations censées dégager des \\ bénéfices pour des secteurs économiques importants, tels \\ que la production pour l'exportation ou les projets \\ d'investissement. \\ Cette licence suppose obligatoirement un lien entre les \\ importations et la production locale.
}




\author{
E140 Licence combinée à ou remplacée par une \\ autorisation spéciale d'importation
}

En plus, ou en remplacement, d'une licence émise par le principal organe habilité (généralement le ministère du Commerce), une autorisation d'importation spéciale ou une inscription dans un registre sont exigées par une autorité spécialisée, chargée de coordonner un secteur de l'économie intérieure (ministère de l'Industrie, ministère de l'Agriculture, etc.).

Cette licence est accordée pour des raisons d'ordre politique ou religieux, mais non économiques.

E150 Licence attribuée pour des raisons non économiques

E151 Licence attribuée pour des raisons politiques

E159 Licence attribuée pour des raisons non économiques, n.d.a.

E190 Licences non automatiques, n.d.a.

E200 Contingents

E210 Contingents globaux

E211 Contingents sans allocation particulière

E212 Contingents alloués aux pays exportateurs

E220 Contingents bilatéraux

E230 Contingents saisonniers

E240 Contingents liés à l'achat de produits d'origine locale

E250 Contingents pour raisons non économiques

E251 Contingents pour raisons politiques

E259 Contingents pour raisons non économiques, n.d.a.

E260 Contingents avec droits de douane

E270 Contingents liés à la production nationale

E290 Contingents, n.d.a.

E300 Interdictions

E310 Interdiction totale (pour des raisons autres que SPS)
Les contingents, ou quotas, supposent la restriction de certains produits à travers la fixation d'un plafond de quantité ou de valeur des biens autorisés à l'importation. Les différentes formes de contingents sont les suivantes :

Les contingents globaux sont établis sur la base de la quantité ou de la valeur totale des importations de produits donnés, et qui peuvent être alloués au premier demandeur, ou bien accordés d'avance à différents fournisseurs.

Contingents accordés au premier demandeur, non répartis entre différents exportateurs.

Contingents répartis d'avance entre les différents exportateurs potentiels.

Contingents d'importations réservés à un pays spécifique Contingents d'importations pour une période de l'année donnée, concernant généralement certains produits agricoles

Contingents définis en pourcentage de la valeur des biens similaires achetés sur le marché local.

Contingents décidés pour des raisons autres qu'économiques.

Contingents motivés par des raisons politiques plutôt qu'économiques.

Système de droits de douane multiples applicables au même produit. Les droits de douane les moins élevés s'appliquent jusqu'à la limite des quotas, tandis les droits les plus élevés concernent les importations au-dessus de cette limite. Le contingent peut être défini en quantité ou en valeur. Lien obligatoire entre les importations (de matières premières ou de produits semi-finis) et la production locale.

Interdiction sans autre condition ni précision. 


\section{E320 Suspension de l'octroi de licences}

E330 Interdiction saisonnière

E340 Interdiction temporaire

E350 Interdiction d'importer en grandes quantités

E360 Interdiction de produits contrevenant aux brevets ou aux droits de propriété intellectuelle E370 Interdictions pour des raisons non économiques

E371 Interdictions pour des raisons religieuses, morales ou culturelles

E372 Interdictions pour des raisons politiques (embargo)

E379 Interdictions pour des raisons non

économiques, n.d.a.

E390 Interdictions, n.d.a.

E400 Mesures quantitatives de sauvegarde

E500 Accord de restriction des exportations

E510 Limitation volontaire des exportations

E511 Accord de contingent

E512 Accord de consultation
La suspension de l'octroi des licences constitue une forme d'interdiction de fait. Cette situation peut survenir dans des cas relatifs à des difficultés temporaires de la balance des paiements, ou pour d'autres raisons.

L'interdiction saisonnière suppose d'interdire les importations pendant une certaine période de l'année ; elle concerne généralement certains produits agricoles.

Cette interdiction n'est mise en place que pour une période limitée, mais ne comporte pas nécessairement de date de fin fixée d'avance. Elle concerne habituellement les questions urgentes.

Obligation d'importer les produits dans des conditionnements ou contenants de taille réduite.

Interdiction des copies, contrefaçons ou imitations de biens protégés par un brevet ou de marque déposée.

Interdictions pour des raisons politiques, religieuses ou autres, mais non économiques.

Certains pays interdiront l'importation, l'utilisation ou la possession de certains produits jugés contraires à leur foi. Ces produits peuvent comprendre des objets non religieux, du porc, des produits alcooliques, des stupéfiants ou tout autre bien qui contreviendrait aux préceptes de leur religion. Tous les produits liés au porc, même s'il ne sont pas alimentaires, comme la peau de porc, demeurent interdits dans certains produits.

Interdiction d'importations en provenance d'un pays ou d'un groupe de pays, pour des raisons politiques.

Mesures ayant un effet sur les restrictions quantitatives. Les mesures quantitatives de sauvegarde sont adoptées lorsque le gouvernement du pays importateur souhaite empêcher ou remédier à des préjudices graves issus de la brusque augmentation des importations, ou pour faciliter les ajustements.

Accord en vertu duquel un exportateur accepte de limiter ses exportations pour éviter d'imposer des restrictions de la part du pays importateur, telles que la mise en place de contingents, de relèvements des tarifs douaniers ou toute autre mesure de contrôle des importations. L'accord peut être conclu entre États ou entre sociétés.

Accord conclu par le gouvernement ou un secteur économique d'un pays exportateur visant à limiter volontairement ses exportations afin d'éviter de subir des restrictions obligatoires des importations par le pays cible. Contingents d'exportation fixés par un pays importateur et acceptés par un pays exportateur pour éviter l'imposition de restrictions obligatoires.

Accord comprenant des clauses de consultation, dans l'optique de mettre en place des restrictions pour certaines circonstances. 


\begin{tabular}{ll}
\hline E513 Accord de coopération administrative & $\begin{array}{l}\text { Accord comprenant des dispositions de coopération } \\
\text { administrative, dans l'optique d'éviter une perturbation des } \\
\text { échanges bilatéraux. }\end{array}$
\end{tabular}

E590 Accord de restriction des exportations, n.d.a.

E900 Mesures de contrôle de la quantité, n.d.a.

F000 MESURES PARATARIFAIRES

F100 Surtaxes douanières

Autres mesures qui accroissent le coût des importations d'une manière analogue aux mesures tarifaires. Les mesures paratarifaires augmentent le prix des importations d'un pourcentage ou d'un montant fixes, calculés respectivement sur la base de la valeur ou de la quantité. On distingue quatre groupes: les surtaxes douanières, les impositions additionnelles, les taxes et impositions intérieures prélevées sur les importations et l'évaluation en douane sur la base d'un prix indicatif.

La surtaxe douanière ou encore « surcharge » ou droit additionnel, est un instrument ad hoc de politique économique destiné à accroître les recettes publiques ou à protéger l'industrie nationale.

F200 Impositions additionnelles

Ces impositions, qui sont prélevées sur les biens importés en sus des droits de douane et des surtaxes douanières et qui n'ont pas d'équivalent interne, comprennent diverses taxes et redevances. Parmi les impositions additionnelles figurent également la taxe sur les transactions en devises, le droit de timbre, la taxe de licence d'importations, les droits de facturation consulaire, l'impôt statistique, l'impôt sur les transports, les charges pour les catégories de produits sensibles. Diverses autres taxes telles que le prélèvement pour la promotion des exportations et les prélèvements destinés à alimenter d'autres fonds spéciaux, l'impôt communal, la taxe d'immatriculation sur les véhicules à moteur importés, la taxe de dédouanement, etc., sont classées comme impositions additionnelles n.d.a.

F210 Taxe sur les transactions en devises F220 Droit de timbre Selon l'article III de l'Accord général sur les tarifs douaniers et le commerce, les taxes internes peuvent s'appliquer aux importations, à conditions que les premières ne soient pas supérieures à celles qui seraient appliquées à des produits F240 Droits de facturation consulaire F250 Impôt statistique F260 Taxe de manutention ou de stockage F270 Impôt sur les transports F280 Charges pour les catégories de produits sensibles F290 Impositions additionnelles, n.d.a.

F300 Taxes et impositions intérieures sur les importations 
F310 Impôt général sur les ventes

F330 Charges pour les catégories de produits sensibles

F390 Taxes et impositions intérieures sur les importations, n.d.a.

\section{F400 Évaluation mercuriale en douane}

F900 Mesures paratarifaires, n.d.a.

G000 MESURES FINANCIÈRES

G100 Paiement anticipé obligatoire

G110 Dépôt préalable à l'importation

G120 Marge de trésorerie obligatoire

G130 Règlement anticipé des droits de douane

G140 Cautions remboursables pour les catégories de produits sensibles

G190 Paiement anticipé obligatoire, n.d.a.
L'impôt général sur les ventes prélevé sur les importations équivaut aux taxes intérieures appliquées à la totalité ou à la plupart des produits. On peut distinguer trois types de taxes intérieures. En premier lieu, l'impôt généralement dénommé " impôt sur les ventes », qui est une imposition ad valorem sur le produit brut des ventes de marchandises, prélevée à intervalles réguliers auprès des commerçants. En deuxième lieu, l'impôt sur le chiffre d'affaires ou taxe en cascade sur les ventes, qui est imposé à plusieurs niveaux de la production et de la distribution, et qui repose sur les recettes brutes, ce qui entraîne une accumulation des prélèvements. En troisième lieu, la taxe sur la valeur ajoutée, qui est un impôt sur le chiffre d'affaires modifié fondé sur la valeur ajoutée nette et non plus sur les recettes brutes, ce qui évite l'accumulation des prélèvements et qui ne modifie ni la structure des prix, ni la répartition des ressources.
Les droits de douane et autres impositions frappant certains produits importés peuvent être prélevés sur la base d'une valeur fixe des marchandises (appelée "valeur mercuriale»). On présente souvent cette pratique comme un moyen d'éviter les fraudes et de protéger l'industrie nationale. La valeur mercuriale transforme de facto un droit ad valorem en un droit spécifique.

Mesures qui réglementent l'accès aux devises destinées aux importations ainsi que leur coût et définissent les conditions de paiement. Elles peuvent accroître le coût des importations à peu près de la même manière que les mesures tarifaires. Paiement anticipé du coût de l'opération d'importation et/ou des droits d'importation connexes, exigé au moment où l'importateur fait sa demande de licence d'importation ou lorsqu'elle lui est octroyée. Ces paiements anticipés obligatoires se répartissent comme suit :

Obligation de déposer un pourcentage de la valeur de la transaction un certain temps avant l'importation, sans que ce dépôt donne droit au versement d'intérêts.

Obligation de déposer le montant total ou une partie de la valeur de la transaction auprès d'une banque commerciale avant l'ouverture d'une lettre de crédit.

Règlement anticipé des droits de douane, en totalité ou en partie, sans que ce règlement donne droit au versement d'intérêts.

Impositions qui sont remboursées quand les produits utilisés ou leurs contenants sont retournés grâce à un système de collecte. 
G200 Taux de change multiples

G300 Allocation restrictive de devises officielles

G310 Interdiction d'allocation de devises

G320 Autorisation de la banque

G330 Licence liée à des devises non officielles

G331 Devises externes

G332 Devise de l'importateur

G339 Licence liée à des devises non officielles, n.d.a.

G390 Allocation restrictive de devises

officielles, n.d.a.

G400 Règlements concernant les conditions de paiement des importations

G500 Délais de transfert, file d'attente

G600 Obligation de cession de devises G900 Mesures financières, n.d.a. H000 MESURES ANTICONCURRENTIELLES

H100 Circuit unique pour les importations

H110 Administration des importations par l'État

H120 Agence d'importation unique

H190 Circuit unique pour les importations,

n.d.a.
Taux de change variables pour les importations en fonction de la catégorie des produits. Habituellement, le taux officiel est réservé aux marchandises de première nécessité. Les autres produits doivent être payés aux tarifs commerciaux ou, occasionnellement, par le biais d'achat de devises aux enchères.

Ces restrictions prennent habituellement la forme de permis, de visas, d'autorisations, etc., dans le but de contrôler les flux d'importation. Il s'agit parfois d'une interdiction d'allocation de devises.

L'allocation de devises officielles pour le paiement des importations est interdite.

La banque centrale doit accorder une autorisation spéciale. Octroi d'une licence en l'absence d'une obligation de devise officielle.

Octroi d'une licence seulement pour les importations nécessitées par des projets d'assistance techniques ou d'autres sources de devises externes.

Octroi d'une licence si les importateurs détiennent des devises dans une banque étrangère.

Règles régissant les conditions de paiement des importations, ainsi que l'octroi et l'utilisation de crédits (étrangers ou nationaux) pour financer ces dernières.

Délai minimal autorisé, entre la date de livraison des marchandises et le règlement final de la transaction d'importation (habituellement 90, 180 ou 360 jours pour les biens de consommation et les facteurs de production industrielle et de deux à cinq ans pour les biens d'équipement). La file d'attente se produit lorsque les délais prescrits ne peuvent être respectés en raison d'une pénurie de devises et que les transactions sont réglées l'une après l'autre à l'issue d'une période d'attente plus longue.

Cession obligatoire de gains en devises à la banque centrale.

Mesures qui accordent des droits exclusifs ou préférentiels à un agent économique ou à un groupe limité d'agents économiques pour des raisons sociales, budgétaires, économiques ou politiques.

Toutes les importations ou celles de certains produits doivent passer par un ou plusieurs organismes ou entreprises d'État. Parfois, le secteur privé peut également recevoir des droits d'importation exclusifs. 
H200 Recours obligatoire à des services nationaux

H210 Recours obligatoire aux compagnies d'assurance nationales

H220 Recours obligatoire aux compagnies de transport nationales

H290 Recours obligatoire à des services nationaux, n.d.a.

H900 Mesures anticoncurrentielles, n.d.a. I000 MESURES APPLICABLES AUX EXPORTATIONS

I100 Taxes à l'exportation

I200 Restrictions quantitatives à l'exportation
I210 Interdiction d'exportation

I220 Contingents d'exportation I230 Obligation de licence ou de permis d'exportation

I240 Enregistrement, réglementation contraignante ou restrictions à l'exportation I290 Restrictions quantitatives à l'exportation, n.d.a.

I300 Certification

I400 Taxe d'inspection

I500 Administration commerciale par l'État

I600 Systèmes de double prix

I900 Mesures à l'exportation, n.d.a. J000 Mesures relatives à l'investissement qui affectent les échanges

J100 Mesures relatives au contenu local

J200 Mesures d'équilibrage des échanges

J900 Mesures relatives à l'investissement qui affectent les échanges, n.d.a.
Droits exclusifs, sanctionnés par l'État, des compagnies nationales d'assurance et de transport concernant la totalité ou une partie des importations.

Les mesures applicables aux exportations sont mises en cuvre par le gouvernement du pays exportateur, pour les biens vendus à l'étranger.

Droits/taxes à l'exportation collectés sur les biens ou les matières premières par le gouvernement du pays exportateur. Les taxes à l'exportation peuvent s'entendre sur une base spécifique ou ad valorem.

Restrictions sur la quantité de biens exportés vers un ou plusieurs pays donné(s), mises en place par le gouvernement du pays exportateur, pour des raisons telles que la pénurie de biens sur le marché domestique, l'évitement de mesures antidumping, ou pour des causes politiques.

Interdiction d'exporter certains produits.

Contingents limitant le volume ou la valeur des exportations.

Les exportateurs doivent obtenir une licence ou un permis octroyé par le gouvernement de leur pays pour pouvoir vendre leurs produits à l'étranger.

Obligation d'enregistrement (à des fins de contrôle) des produits avant leur exportation.

Obligation par le pays exportateur d'obtenir une certification sanitaire, phytosanitaire ou autre avant l'exportation des biens.

Taxe prélevée par l'autorité gouvernementale du pays exportateur visant à couvrir les frais d'inspection des produits destinés à la vente à l'étranger.

Tout ou partie des exportations de certaines matières premières doivent transiter par certaines entreprises spécifiées par l'État.

Application de prix différents aux produits en fonction de leur marché final (national ou exportation).

Obligation d'intégrer un niveau minimum de composants produits localement, ce qui restreint l'usage de composants importés.

Mesures limitant l'acquisition ou l'usage des produits importés par une entreprise à un niveau en rapport avec le volume ou la valeur des produits locaux qu'elle exporte. 


\section{KO00 RESTRICTIONS DE DISTRIBUTION* \\ LO00 RESTRICTIONS SUR LES SERVICES POSTÉRIEURS À LA VENTE*}

M000 SUBVENTIONS*

NOO0 RESTRICTIONS APPLICABLE AUX
FOURNISSEURS DE L'ETAT*

O000 PROPRIÉTÉ INTELLECTUELLE*
Dispositions visant à limiter et à réglementer la distribution des produits. Peuvent s'y ajouter des obligations de licence ou de certification supplémentaires.

Mesures limitant les possibilités des producteurs de biens exportés de proposer des services postérieurs à la vente dans le pays importateur.

Contribution financière d'un gouvernement ou d'un organe gouvernemental à une structure de production, concernant un secteur ou une entreprise donnés, telle qu'un transfert direct effectif ou potentiel de fonds (par ex. dotations, prêts, apports de capital), des paiements à un mécanisme de financement ou un soutien aux revenus ou aux prix.

Mesures de contrôle de l'acquisition de biens par des agences gouvernementales, généralement plutôt auprès de fournisseurs nationaux.

La législation sur la propriété intellectuelle couvre les brevets, les marques déposées, les dessins et modèles industriels, la conception structurelle des circuits intégrés, les droits d'auteur, les indications géographiques et les secrets d'affaires.

P000 RĖGLES D'ORIGINE*

Les règles d'origine comprennent les lois, réglementations et résolutions administratives de portée générale appliquées par le gouvernement de pays importateurs pour déterminer quel est le pays d'origine des biens. Les règles d'origine peuvent limiter les échanges commerciaux lorsqu'il est difficile de déterminer l'origine du produit final (par exemple si les matières premières et les produits semi-finis proviennent de différents pays). Les règles d'origine jouent un rôle important dans la mise en place d'instruments de politique commerciale tels que les droits antidumping et compensatoires, le marquage de l'origine et les mesures de sauvegarde.

* Tant qu'une autre décision dans ce sens n'aura pas été prise par les membres de l'équipe MAST, il ne sera effectué aucun effort de collecte des mesures appartenant à ces catégories auprès de sources officielles. En effet, ces catégories ont été créées pour rendre compte des préoccupations éventuelles des commerçants par le biais d'enquêtes et de questionnaires.

\section{Classification des obstacles procéduraux}

\section{A : CARACTÈRE ARBITRAIRE OU INCOHÉRENCES}

1. Comportement des pouvoirs publics

2. Classification et/ou évaluation des produits

3. Application des procédures, réglementations ou obligations (y compris incohérences entre les procédures ou réglementations locales et nationales).

\section{B : COMPORTEMENT DISCRIMINATOIRE FAVORISANT CERTAINS PRODUCTEURS OU FOURNISSEURS}

1. Fournisseurs ou producteurs locaux du marché cible

2. Fournisseurs issus d'autres pays

3. Grandes (ou petites) entreprises

\section{C : INEFFICACITÉ OU OBSTRUCTION}

1. Exigences excessives de production de documents

2. Essais, certification ou étiquetage stricts/détaillés/redondants

3. Délais administratifs (par ex. pour une autorisation ou une approbation)

4. Systèmes d'approbation complexes (par ex. plusieurs entités doivent exprimer leur accord) 
5. Délais de soumission trop courts pour les informations ou formulaires requis

6. Procédures dépassées (par ex. absence d'automatisation)

7. Ressources insuffisantes (par ex. sous-effectifs, équipements insuffisants sur le marché cible)

\section{D : OPACITÉ}

1. Information inadaptée sur les lois/la réglementation/l'enregistrement

2. Changement sans préavis de procédure, de réglementation ou de contrainte

3. Absence de point d'information

4. Processus opaque d'appel d'offres ou de remboursement de la part du gouvernement

5. Résolution opaque des litiges

6. Obligation ou incitation à des paiements informels

\section{E : PROBLÈMES JURIDIQUES}

1. Absence de mise en application de la loi (par ex. concernant les brevets, les droits d'auteur, les marques déposées, la confidentialité)

2. Résolution des litiges / processus d'appel /respect des garanties données par la loi inappropriés

3. Infrastructures juridiques inadaptées

\section{F : FRAIS OU TAXES EXCEPTIONNELLEMENT ÉLEVÉS (par ex. droits de timbre, essais ou autres services rendus)}




\section{Annexe 2}

\section{Dérivation des équilibres de marché et des mesures du bien-être}

Cette annexe détaille la dérivation des fonctions de demande du consommateur et fournit les expressions analytiques nécessaires au calcul des effets de bien-être pour les différents cas évoqués dans le corps de la présente étude.

Fonction de demande inverse pour les consommateurs indifférents et concernés

Rappel de la fonction d'utilité (1) :

$U_{i}\left(q_{i}, w_{i}\right)=a q_{i}-\bar{b} q_{i}^{2} / 2-\operatorname{Ir}_{i} q_{i}+w_{i}$

La maximisation de (1) sous la contrainte budgétaire $p q_{i}+w_{i}=y_{i}$, sachant que $y_{i}$ représente le revenu de la personne $i$, aboutit à fonction de demande inverse suivante $: p=a-\bar{b} q_{i}-I \times r_{i}$. La demande correspondante pour le consommateur $i$ est égale à $q_{i}(p)=\left(a-p-I \times r_{i}\right) / \bar{b}$. La demande globale pour tous les consommateurs est donc $Q=\sum_{i=1}^{N} q_{i}(p)$, ce qui aboutit à $Q=N(a-p) / \bar{b}-I \times \sum_{i=1}^{N} r_{i} / \bar{b}$. En supposant que $b=\bar{b} / N$, l'inversion de la demande globale aboutit à la fonction de demande inverse

$$
p^{D}(Q, I)=a-b Q-\left(I \times \sum_{i=1}^{N} r_{i}\right) / N .
$$

Nous prenons pour hypothèse qu'une proportion $\beta=N_{l} / N$ de consommateurs sont complètement indifférents à la caractéristique spécifique, avec $r_{i}=0$ pour chaque $i=1, . ., N_{l}$. La proportion $(1-\beta)=1$ $N_{l} / N$ de consommateurs préfère ne pas consommer la caractéristique spécifique et associe un préjudice par unité consommée égal à $r_{2}$ pour tout ce sous-groupe. Dans ce cas, il est possible de diviser la demande globale précédente en deux sous-groupes, à savoir $Q_{1}^{D}(p)=\sum_{i=1}^{N_{1}} q_{i}(p)=N_{1}(a-p) / \bar{b}$ pour les consommateurs indifférents à la caractéristique négative, et $Q_{2}^{D}(p, I)=\sum_{i=1}^{\left(N-N_{1}\right)} q_{i}(p)=\left(N-N_{1}\right)\left[a-p-I \times r_{2}\right] / \bar{b}$ pour les consommateurs concernés. Avec $b=\bar{b} / N, N_{l}=\beta N$ et $\left(N-N_{l}\right)=(1-\beta) N$, les demandes inverses respectives sont représentées par (2) dans le corps de l'étude.

\section{Bien-être}

Pour chacune des configurations, le bien-être domestique est calculé à partir des zones de surplus associées à l'offre et à la demande et aux externalités lorsqu'elles surviennent. 


\section{Externalités de consommation liées directement à celle-ci}

\section{Norme prohibitive (interdiction d'importation)}

Les expressions analytiques au point d'équilibre en situation d'autarcie (avec interdiction de commerce) sont les suivantes :

$$
\begin{aligned}
p^{A} & =\frac{a c_{O}}{c_{O}+b M_{O}} \text { pour le prix, } \\
Q^{A} & =\frac{a M_{O}}{c_{O}+b M} \text { pour la quantité, }
\end{aligned}
$$

$P S^{A}=\int_{0}^{Q^{A}}\left[p^{A}-p_{O}^{S}(Q)\right] d Q=\frac{a^{2} c_{O} M_{O}}{2\left(c_{O}+b M_{O}\right)^{2}}$ pour l'ensemble des profits des producteurs nationaux,

$C S^{A}=\int_{0}^{Q^{A}}\left[p^{D}(Q)-p^{A}\right] d Q=\frac{a^{2} b M_{O}{ }^{2}}{2\left(c_{O}+b M_{O}\right)^{2}}$ pour le surplus du consommateur,

$$
W^{A}=P S^{A}+C S^{A}=\frac{a^{2} M_{O}}{2 c_{O}+2 b M_{O}} \text { pour le bien-être. }
$$

\section{Libre-échange}

Les expressions analytiques au point d'équilibre dans la situation de libre-échange sont les suivantes :

$p^{B}=\frac{c_{O} c_{F}\left(a-(1-\beta) r_{2}\right)}{c_{O}\left(c_{F}+b M_{F}\right)+b M_{O} c_{F}}$ pour le prix,

$Q^{B}=\frac{\left(c_{O} M_{F}+c_{F} M_{O}\right)\left(a-(1-\beta) r_{2}\right)}{c_{O}\left(c_{F}+b M_{F}\right)+b M_{O} c_{F}}$ pour la quantité,

$P S_{O}^{B}=\int_{0}^{q_{O}^{B}}\left[p^{B}-p_{O}^{S}(Q)\right] d Q=\frac{c_{O}\left(c_{F}\right)^{2} M_{O}\left(a-(1-\beta) r_{2}\right)^{2}}{2\left[c_{O}\left(c_{F}+b M_{F}\right)+b M_{O} c_{F}\right]^{2}}$ pour l'ensemble des profits des

producteurs nationaux,

$$
C S_{1}^{B}=\int_{0}^{q_{1}^{B}}\left[p_{1}^{D}(Q)-p^{B}\right] d Q=\frac{\beta\left(a b\left(c_{O} M_{F}+c_{F} M_{O}\right)+c_{O} c_{F}(1-\beta) r_{2}\right)^{2}}{2 b\left[c_{O}\left(c_{F}+b M_{F}\right)+b M_{O} c_{F}\right]^{2}}, \text { pour le surplus } \mathrm{du}
$$

consommateur indifférent,

$$
C S_{2}^{B}=\int_{0}^{q_{2}^{B}}\left[p_{2}^{D}(Q, 1)-p^{B}\right] d Q=\frac{(1-\beta)\left(a b\left(c_{O} M_{F}+c_{F} M_{O}\right)-r_{2}\left(b\left(c_{O} M_{F}+c_{F} M_{O}\right)+\beta c_{O} c_{F}\right)\right)^{2}}{2 b\left[c_{O}\left(c_{F}+b M_{F}\right)+b M_{O} c_{F}\right]^{2}},
$$

pour le surplus du consommateur concerné,

$W^{B}=P S_{O}^{B}+C S_{1}^{B}+C S_{2}^{B}$ pour le bien-être national,

$P S_{F}^{B}=\int_{0}^{q_{F}^{B}}\left[p^{B}-p_{F}^{S}(Q)\right] d Q=\frac{c_{F}\left(c_{O}\right)^{2} M_{F}\left(a-(1-\beta) r_{2}\right)^{2}}{2\left[c_{O}\left(c_{F}+b M_{F}\right)+b M_{O} c_{F}\right]^{2}}$ pour l'ensemble des profits des

producteurs étrangers. 


\section{Libre-échange avec étiquetage obligatoire}

Les expressions analytiques au point d'équilibre dans la situation de libre-échange avec étiquetage obligatoire sont les suivantes :

$p_{1}^{L}=\frac{a c_{F} \beta}{c_{F} \beta+b M_{F}}$ pour le premier prix,

$Q^{1}=\frac{a M_{F} \beta}{c_{F} \beta+b M_{F}}$ pour la première quantité,

$C S_{1}^{L}=\int_{0}^{Q^{1}}\left[p_{1}^{D}(Q)-p_{1}^{L}\right] d Q=\frac{a^{2} b M_{F}^{2} \beta}{2\left(c_{F} \beta+b M_{F}\right)^{2}}$, pour le surplus du consommateur indifférent,

$P S_{F}^{L}=\int_{0}^{Q^{1}}\left[p_{1}^{L}-p_{F}^{S}(Q)\right] d Q=\frac{a^{2} c_{F} M_{F} \beta^{2}}{2\left(c_{F} \beta+b M_{F}\right)^{2}}$ pour l'ensemble des profits des producteurs étrangers.

$p_{2}^{L}=\frac{a c_{O}(1-\beta)}{c_{O}(1-\beta)+b M_{O}}$ pour le second prix,

$Q^{2}=\frac{a M_{O}(1-\beta)}{c_{O}(1-\beta)+b M_{O}}$ pour la seconde quantité,

$C S_{2}^{L}=\int_{0}^{Q^{2}}\left[p_{2}^{D}(Q, 0)-p_{2}^{L}\right] d Q=\frac{a^{2} b M_{O}^{2}(1-\beta)}{2\left(c_{O}(1-\beta)+b M_{O}\right)^{2}}$, pour le surplus du consommateur concerné,

$P S_{O}^{L}=\int_{0}^{Q^{2}}\left[p_{2}^{L}-p_{O}^{S}(Q)\right] d Q=\frac{a^{2} c_{O} M_{O}(1-\beta)^{2}}{2\left(c_{O}(1-\beta)+b M_{O}\right)^{2}}$ pour l'ensemble des profits des producteurs nationaux,

$W^{L}=P S_{O}^{L}+C S_{1}^{L}+C S_{2}^{L}$ pour le bien-être national,

\section{Externalité de production}

Les expressions analytiques au point d'équilibre dans la situation de libre-échange sont les suivantes :

$p^{G}=\frac{a c_{O} c_{F}}{c_{O}\left(c_{F}+b M_{F}\right)+b M_{O} c_{F}(1-\lambda)}$ pour le prix attendu,

$Q^{G}=\frac{a\left(c_{O} M_{F}+c_{F}(1-\lambda) M_{O}\right)}{c_{O}\left(c_{F}+b M_{F}\right)+b M_{O} c_{F}(1-\lambda)}$ pour la quantité,

$P S_{O}^{G}=\int_{0}^{q_{O}^{G}}\left[p^{G}-p_{O}^{S}(Q)\right] d Q=\frac{a^{2} c_{O}\left(c_{F}\right)^{2}(1-\lambda)^{2} M_{O}}{2\left[c_{O}\left(c_{F}+b M_{F}\right)+b M_{O} c_{F}(1-\lambda)\right]^{2}}$ pour l'espérance de profit globale

des producteurs nationaux,

$C S^{G}=\int_{0}^{Q^{G}}\left[p^{D}(Q)-p^{B}\right] d Q=\frac{b\left(a\left[c_{O} M_{F}+c_{F}(1-\lambda) M_{O}\right]\right)^{2}}{2\left[c_{O}\left(c_{F}+b M_{F}\right)+b M_{O} c_{F}(1-\lambda)\right]^{2}}$, pour le surplus $d u$

consommateur,

$W^{G}=P S_{O}^{G}+C S^{G}$ pour l'espérance de bien-être national, 
$P S_{F}^{G}=\int_{0}^{q_{F}^{G}}\left[p^{G}-p_{F}^{S}(Q)\right] d Q=\frac{a^{2} c_{F}\left(c_{O}\right)^{2} M_{F}}{2\left[c_{O}\left(c_{F}+b M_{F}\right)+b M_{O} c_{F}(1-\lambda)\right]^{2}}$ pour l'espérance de profit globale des producteurs étrangers.

\section{Externalité des ressources communes mondiales}

Les expressions analytiques au point d'équilibre dans la situation de libre-échange sont les suivantes :

$p^{E}=\frac{a c_{O} c_{F}}{c_{O}\left(c_{F}+b M_{F}\right)+b M_{O} c_{F}}$ pour le prix,

$Q^{E}=\frac{a\left(c_{O} M_{F}+c_{F} M_{O}\right)}{c_{O}\left(c_{F}+b M_{F}\right)+b M_{O} c_{F}}$ pour la quantité,

$P S_{O}^{E}=\int_{0}^{q_{O}^{E}}\left[p^{E}-p_{O}^{S}(Q)\right] d Q=\frac{a^{2} c_{O}\left(c_{F}\right)^{2} M_{O}}{2\left[c_{O}\left(c_{F}+b M_{F}\right)+b M_{O} c_{F}\right]^{2}}$ pour l'ensemble des profits des

producteurs nationaux,

$C S^{E}=\int_{0}^{Q^{E}}\left[p^{D}(Q)-p^{B}\right] d Q=\frac{b\left(a\left(c_{O} M_{F}+c_{F} M_{O}\right)\right)^{2}}{2\left[c_{O}\left(c_{F}+b M_{F}\right)+b M_{O} c_{F}\right]^{2}}$, pour le surplus du consommateurs,

$E X=r_{2}(1-\beta) Q^{E}=r_{2} Q_{2}^{E}=\frac{r_{2}(1-\beta) a\left(c_{O} M_{F}+c_{F} M_{O}\right)}{c_{O}\left(c_{F}+b M_{F}\right)+b M_{O} c_{F}}$, pour l'externalité

$W^{E}=P S_{O}^{E}+C S^{E}-E X$ pour le bien-être national,

$$
P S_{F}^{E}=\int_{0}^{q_{F}^{E}}\left[p^{E}-p_{F}^{S}(Q)\right] d Q=\frac{a^{2} c_{F}\left(c_{O}\right)^{2} M_{F}}{2\left[c_{O}\left(c_{F}+b M_{F}\right)+b M_{O} c_{F}\right]^{2}} \text { pour l'ensemble des }
$$

profits des producteurs étrangers. 